\title{
XXIII.-On Systems of Solutions of Homogeneous and Central Equations of the nth Degree and of two or more Variables; with a Discussion of the Loci of such Equations. By the Hon. Lord M'Laren. (Plates I.-VI.)
}

(Read 6th July 1888.)

\begin{tabular}{|c|c|c|c|}
\hline & & & \\
\hline$a$ & & 13. Diameters in Central Curves of Higher Degree & 1068 \\
\hline & 1044 & 14. S & \\
\hline Apt & & rves re- & \\
\hline Ho & 1045 & & 1070 \\
\hline 3. Solu & & diaxial Curves, - & 1072 \\
\hline 4. Anot & 104 & Curv & 1 \\
\hline & 1050 & $14 d$. & 10 \\
\hline $\begin{aligned} \text { 5. Solu } \\
\text { in } \\
\text { 6. Solu }\end{aligned}$ & 1 & $\begin{array}{l}\text { 15. Deternination of Contour-Lines of Homogeneous } \\
\text { Surfaces, . } \\
\text { 16. Central Curves whose Equations are of the Form }\end{array}$ & 10 \\
\hline $\begin{array}{l}\text { of Homogeneous Equations of } \mathrm{E} \\
\text { e Variables. (Case IV.), . }\end{array}$ & 105 & $\begin{array}{l}\mathrm{F}_{1}(x, y)^{n}=\mathrm{F}_{2}(x, y)^{n-p}, \\
\text { Examples of such Curves (Sixth Degree), } \\
\text { ontour- Lines of Surfaces derived from Central }\end{array}$ & 31 \\
\hline $\begin{array}{l}\text { 8. To fij } \\
\text { of }\end{array}$ & & sing through their Centres, & \\
\hline 9. Clas & & $\begin{array}{l}\text { One Axis, } \\
\text { pression for the Oval of }\end{array}$ & 108 \\
\hline on of Central Curves of the $F$ & & 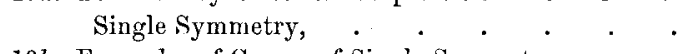 & \\
\hline & & 0 & 089 \\
\hline & 10 & Forms, & 090 \\
\hline $\mathrm{F}_{0}$ & 10 & 1. Biradial Coordinates, & 109 \\
\hline
\end{tabular}

The purpose of the present paper is to ascertain how far it is possible to find exact solutions or values of $x, y, \& c$, in equations between variables, so that the forms of plane curves and contour-lines of surfaces may be exactly determined. No approximate methods have been admitted, and only those methods have been used which are applicable to algebraic equations of every degree and any number of variables. In the examples given I have generally selected equations of even degree and even powers of the variables. But every such solution evidently includes the solution of the non-central equation of half the degree having corresponding terms and equal coefficients. The methods of solution employed are founded on the following introductory theorem or principle, which may be described as that of Homogeneous or Linear Variation of the quantities.

The paper, as laid before the Royal Society in July 1888, embraced only the solution of homogeneous equations in which one of the quantities was given explicitly in terms of the others. The preparation of the paper for the press having been interrupted by my absence abroad for a considerable time, I have resumed the investigation from a more general point of view. 


\section{Principle of Homogeneous or Linear Variation of a Function.}

If $\mathrm{F}(\alpha, \beta, \gamma \ldots)^{n}=0$ be a homogeneous function of the $n^{\text {th }}$ degree of any number of quantities, $\alpha, \beta, \gamma, \ldots$; and if $\alpha_{1}, \beta_{1}, \gamma_{1}$, \&c., be known values of these quantities satisfying the equation, then may another set of values, $a_{2}, \beta_{2}, \gamma_{2}$, satisfying the equation, be found by multiplying or dividing each term by any desired factor, $m^{n}$.

Let the function consist of a series of homogeneous terms of powers of the quantities $\alpha, \beta, \gamma, \ldots$ multiplied by coefficients $p_{1} q_{1} \ldots$ and equated to zero. Let $\alpha_{1}, \beta_{1}, \gamma_{1}$ be values satisfying the equation; which accordingly will be of the form

$$
\alpha^{n}+p_{1} \alpha_{1}^{n-1} \beta_{1}+p_{2} \alpha_{1}^{n-1} \beta^{2}+\ldots q_{0} \beta_{1}^{n}+q_{1} \beta_{1}^{n-1} \gamma_{1}+q_{2} \beta_{1}{ }^{n-2} \gamma_{1}^{2}+\ldots+r_{r} \alpha_{1}^{n-p-q} \beta_{1}^{p} \gamma_{1}^{q}+\ldots=0 ;
$$

where the last term is the generalised term for three quantities.

To find a new series of values satisfying the equation, we have only to multiply every term by the same numerical quantity, $m^{n}$. The equation is, of course, unaltered in value, and is now of the form

$$
\begin{gathered}
\left(m \alpha_{1}\right)^{n}+p_{1}\left(m \alpha_{1}\right)^{n-1}\left(m \beta_{1}\right)+p_{2}\left(m \alpha_{1}\right)^{n-2}(m \beta)^{2}+\ldots q_{0}\left(m \beta_{1}\right)^{n}+q_{1}\left(m \beta_{1}\right)^{n-1}\left(m \gamma_{1}\right) \\
+r_{r}\left(m \alpha_{1}\right)^{n-p-q}\left(m \beta_{1}\right)^{p}\left(m \gamma_{1}\right)^{q}+\ldots=0,
\end{gathered}
$$

where the term in the second line is the generalised form of a term resulting from the multiplication of the function by $m^{n}$.

By writing $\alpha_{2}$ for $m a_{1}, \beta_{2}$ for $m \beta_{1}, \gamma_{2}$ for $m \gamma_{1}$, \&c., the equation is restored to its original form, with a new set of values, $a_{2}, \beta_{2}, \gamma_{2}$, of these indeterminate quantities satisfying the equation. Comparing the two sets of values, we find the relation

which was to be proved.

$$
\frac{\alpha^{2}}{\alpha_{1}}=\frac{\beta^{2}}{\beta_{1}}=\frac{\gamma_{2}}{\gamma_{1}}=m
$$

The preceding proof evidently includes the cases of negative, reciprocal, and fractional indices.

In the preceding theorem it is not assumed that all the quantities $\alpha, \beta, \gamma, \ldots$ are variables; and the proof is evidently the same, whether all the quantities are conceived as being subject to indefinite variation, or whether some of them are conceived as having only certain definite values from which values of the other quantities are to be obtained. For example, if $a_{1} \beta_{1}$ are variable coordinates, and $\gamma_{1}$ is a parameter, the set of values $\alpha_{1}, \beta_{1}, \gamma_{1}$ represents a point on a plane curve of the $n$th degree having the parameter $\gamma_{1}$, and the set of values $\alpha_{2}, \beta_{2}, \gamma_{2}$ represents a corresponding point on a similar curve whose parameter is $\gamma_{2}$. But if the three quantities $a, \beta, \gamma$ are all conceived as being subject to indefinite variation, $\gamma$ being then a third coordinate, the function represents a conical surface of the $n$th degree, and the two sets of values then represent corresponding points on parallel, and therefore similar, plane sections of this surface.

Again, certain of the quantities may represent the coordinates of a point on a central 
plane curve or central surface, while others of these quantities may represent the coordinates of the centre. If now the function represents a central surface whose centre is variable in position, we may have seven quantities, whereof one is a parameter, and six are variables. When the number of variables exceeds three there must, of course, be other relations between the coordinates, otherwise the problem becomes indeterminate. In the case supposed, a second equation between the coordinates of the centre and one of the coordinates of the surface determines the curve or surface which the centre is supposed to describe, and supplies the necessary elements for the solution of the first equation. I have introduced this illustration because every homogeneous equation of even degree of three or four quantities represents a central curve or central surface respectively referred to the centre; and it is easily seen that, if the origin be changed to any point, whether exterior or interior to the curve, the left-hand side of the resulting equation is a homogeneous function of the original coordinates, and the coordinates of the centre.

\section{Application of the Principle to finding Solutions of Homogeneous Equations of one part. (Case I.)}

The most obvious application of the method of homogeneous variation is to the exact determination of a series of points on a curve or surface whose equation is given in the form of a homogeneous function equated to an arbitrary term. The method, however, is purely analytical, and it is not necessary that the quantities should have a geometrical interpretation. The arbitrary term is to be expressed as the $n^{\text {th }}$ power of a number $w$, and the equation is then of the form-

$$
x^{n}+\mathrm{A}_{\mathbf{1}} x^{n-1} y+\mathrm{A}_{2} x^{n-2} y^{2}+\ldots+\mathbf{A}_{n} y^{n}=w^{n} .
$$

The quantity $w$ is evidently a parameter, being the value of $x$ when $y=0$. It is required to find a series of exact values of $x$ and $y$ to the given parameter $w$. The values to be found may be denoted by $x_{1}, y_{1}, x_{2}, y_{2} \ldots$ Let $\xi_{1}, \eta_{1}$ be any values arbitrarily assumed; these values are to be inserted in the given function, and the value of the parameter computed by summing the terms and extracting the $n$th root of the sum. The equation formed may be called auxiliary equation (1); and may be written-

$$
\xi_{1}^{n}+\mathrm{A}_{1} \xi_{1}^{n-1} \eta_{1}+\mathrm{A}_{2} \xi_{1}^{n-2} \eta_{1}^{2}+\ldots+\mathrm{A}_{n} \eta_{1}^{n}=w_{1}^{n} .
$$

Then by the preceding theorem we have the relation $x_{1} / \xi_{1}=y_{1} / \eta_{1}=w / w_{1}$, which gives for the coordinates of the first point (or first set of values of the original equation) $x_{1}=\xi_{1} w / w_{1} ; y_{1}=\eta_{1} w / w_{1}$.

A second auxiliary equation being formed from new assumed values $\xi_{2} \eta_{2}$, and the parameter $w_{2}$ computed, we find from these data the coordinates of a second point (or second set of values of the original equation), viz., $x_{2}=\xi_{2} w / w_{2} ; y_{2}=\eta_{2} w / w_{2}$, and so on. These are true algebraic solutions of the given equation. 
This method of finding solutions of indeterminate quantities is hereafter referred to as the method of Homogeneous Variation, because all the quantities are varied proportionately in order to obtain a new series of values.

Although only two variables, $x$ and $y$, are here expressed, the explanation of the method of solution is intended to cover the case of an equation of three or more variables. In order to simplify the illustrations as much as possible, I shall gemerally suppose two variable quantities $x$ and $y$; or $r \cos \theta$ and $r \sin \theta$; $w$ is then the parameter or intercept on the axis of $X$. It is convenient to take this quantity =unity, which can always be done by dividing out.

In order that the series of points to be found may correspond to equal angular intervals, it is best to assume $\xi_{1}$.. and $\eta_{1}$.. equal respectively to the cosine and sine of an angle. Then $x_{1} \ldots y_{1}$. are proportional to the same cosine and sine, and are the rectangular coordinates of the curve to the argument $\theta$.

Example 1.

$$
x^{4}+2 x^{3} y+3 x^{2} y^{2}+4 x y^{3}+2 y^{4}=w^{4}=1 .
$$

For the sake of clearness, I shall, in this example only, dispense with the use of Ingarithmic tables, and find two values of $x$ and $y$ from auxiliary equations in which the assumed values are whole numbers.

(1) Let $\xi_{1}=1 ; \eta_{1}=1$. The sum of the terms of the auxiliary equation is 12 ; $\therefore w_{1}=12^{1 / 4} ; x_{1}=\xi / w_{1}=\frac{1}{12^{1 / 4}}=y_{1}$.

This may be verified as follows:-Let $x_{1}$ and $y_{1}$ have the values here found. Then taking the terms of the equation in their order,

$$
x^{4}=\left(\frac{1}{12^{1 / 4}}\right)^{4}=\frac{1}{12} ; 2 x^{3} y=2\left(\frac{1}{12^{1 / 4}}\right)^{3}\left(\frac{1}{12^{1 / 4}}\right)=\frac{2}{12},
$$

and so on; and the terms are as under-

\begin{tabular}{|c|c|c|c|c|}
\hline$x^{4}$ & $2 x^{3} y$ & $3 x^{2} y^{2}$ & $4 x y^{3}$ & $2 y^{4}$ \\
\hline$\frac{1}{12}$ & $\frac{2}{12}$ & $\frac{3}{12}$ & 4 & $\frac{2}{12}$ \\
\hline
\end{tabular}

Sum of the terms $=\frac{1+2+3+4+2}{12}=1$, as it should be.

(2) Let $\xi_{2}=1 ; \eta_{2}=2$; the sum of the terms of the auxiliary equation is

$$
1+4+12+32+32=81 ; \therefore w_{2}=81^{1 / 4}=3 ; x_{2}=\xi_{2} / w_{2}=\frac{1}{3} ; y_{2}=\eta / w_{2}=\frac{2}{3} .
$$

This solution may be verified in the same way as the preceding without the use of logarithms. 
In the next example I shall take the assumed quantities $\xi_{1,2} \ldots \eta_{1,2} \ldots$ from the trigonometrical tables; so that $\log \xi_{1}=\log \cos \theta_{1} ; \log \eta_{1}=\log \sin \theta_{1} ;$ and so on, and thence determine $x_{1,2} \ldots y_{1,2} \ldots$ for parameter $=1$.

Example 2.

$$
x^{10}+x^{8} y^{2}+x^{6} y^{4}+y^{10}=w^{10}=1 .
$$

Values of $x$ and $y$ will be found to the arguments, $\theta=20^{\circ} ; \theta=30^{\circ}$.

1. Let $\theta_{1}=20^{\circ} ; \xi_{1}=\cos 20^{\circ} ; \eta_{1}=\sin 20^{\circ} ; x_{1}=\xi_{1} / w_{1} ; y_{1}=\eta_{1} / w_{1}$.

$$
\begin{aligned}
& \log \cos 20^{\circ}=\overline{1} \cdot 9730 \\
& \begin{array}{ll}
\log \cos ^{6} \quad \overline{1} 8380
\end{array} \\
& \log \cos ^{8} \quad \overline{1} \cdot 7840 \\
& \log \cos ^{10} \quad \overline{\mathbf{1}} \cdot 7300 \\
& \text { Nat. numbers. } \\
& \log \cos ^{8}=1 \cdot 7840 \\
& \log \sin ^{2}+\overline{1} \cdot 0682 \\
& \xi^{8} \eta^{2}=0.0712 \\
& \xi^{10} \quad 0.5370 \\
& 2 \cdot 8522 \\
& \log \sin 20^{\circ}=\overline{1} \cdot 5341 \\
& \log \sin ^{2} \quad \overline{\mathbf{1}} \cdot 0682 \\
& \log \sin ^{4} \quad \overline{2} \cdot 1364 \\
& \xi^{6} \eta^{4} \quad 0.0094 \\
& =\log \xi \eta^{2} \\
& \log \cos ^{6}=1 \cdot 8380 \\
& \log \sin ^{4}+\overline{\mathbf{1}} \cdot 1364 \\
& \log \sin ^{10} \quad \overline{5} \cdot 3410 \\
& \eta^{10} \text { (insensible) } \\
& \log w^{10}=1 \cdot 7907 \\
& \log w \quad 1.9791 \\
& \overline{w^{10}=0.6176} \quad=\log \xi^{6} \eta^{4} \\
& \log \eta_{1}=1.5341 \\
& -\log w_{1} \quad 1.9791 \\
& \log y_{1} \overline{1.5550}
\end{aligned}
$$

2. Let $\theta=30^{\circ} ; \xi_{2}=\cos 30^{\circ} ; \eta_{2}=\sin 30^{\circ} ; x_{2}=\xi_{2} / w_{2} ; y_{2}=\eta_{2} / w_{2}$.

$$
\begin{aligned}
& \log \cos 30^{\circ}=\overline{1} \cdot 9375 \\
& \log \cos ^{6} \quad \overline{\mathbf{1}} \cdot 6250 \\
& \log \cos ^{8} \quad \bar{I} \cdot 5000 \\
& \log \cos ^{10} \quad \overline{\mathbf{1}} \cdot 3750 \\
& \log \sin 30^{\circ} \quad \overline{\mathrm{l}} \cdot 6990 \\
& \log \sin ^{2} \quad \overline{\mathbf{1}} \cdot 3980 \\
& \log \sin ^{4} \quad \overline{\mathbf{2}} \cdot 7960 \\
& \log \sin ^{10} \quad \overline{4} \cdot 9900 \\
& \log w^{10} \quad 1.5348 \\
& \log w \quad 1.9534 \\
& \text { Nat. numbers. } \\
& \log \cos ^{8}=1 \cdot 5000 \\
& \log \sin ^{2}+1 \cdot 3980 \\
& \xi^{8} \eta^{2}=0.0791 \\
& \overline{2} \cdot 8980 \\
& \xi^{1} \quad 0 \cdot 2371 \\
& =\log \xi^{8} \eta^{2} \\
& \log \cos ^{6} \quad \overline{\mathbf{1}} \cdot 6250 \\
& \log \sin ^{4}+\overline{2} \cdot 7960 \\
& \xi^{6} \eta^{4} \quad 0.0264 \\
& \overline{2} \cdot 4210 \\
& \boldsymbol{\eta}^{10} \text { (insensible) } \\
& w^{10}=0.3426 \\
& =\log \xi^{6} \eta^{4} \\
& \log \xi_{2}=1.9375 \\
& \text { - } \log w_{2} \quad 1.9534 \\
& \log \eta_{2}=1 \cdot 6990 \\
& \log x_{2} \overline{\mathbf{1 . 9 8 4 1}} \\
& \log w_{2} \quad 1.9534 \\
& \log y_{2} \overline{\mathbf{1 \cdot 7 4 5 6}} \\
& \text { Results }\left\{\begin{array}{l}
x_{1}=\cdot 9860 ; y_{1}=\cdot 3589 ; \\
x_{2}=\cdot 9640 ; y_{2}=\cdot 5567 .
\end{array}\right.
\end{aligned}
$$

The following independent analytical proof of the general theorem, including its 
extension to any number of variable quantities, was communicated to me by Dr Thомаs MUIR after reading the first sketch of this paper:-

Since $x=a, y=b$ is manifestly a solution of the equation

$$
\mathrm{A}_{0} x^{n}+\mathrm{A}_{1} x^{n-1} y+\ldots+\mathrm{A}_{n} y^{n}=\mathrm{A}_{0} a^{n}+\mathrm{A}_{1} a^{n-1} b+\ldots+\mathrm{A}_{n} b_{\mu},
$$$$
=p \text { say, }
$$

then

is a solution of the equation

$$
x=\frac{a}{p_{\frac{1}{n}}^{1}}, \quad y=\frac{b}{p_{n}^{1}}
$$

$$
\mathrm{A}_{0} x^{n}+\mathrm{A}_{1} x^{n-1} y+\ldots+\mathrm{A}_{n} y^{n}=1 .
$$

For, substituting $a / p^{\frac{1}{n}}, b / p^{\frac{1}{n}}$ for $x, y$, the left-hand side becomes

$$
\mathrm{A}_{0} \cdot \frac{a^{n}}{p}+\mathrm{A}_{1} \frac{a^{n-1} b}{p}+\ldots+\mathrm{A}_{n} \frac{b^{n}}{p} ; \text { i.e., } \frac{p}{p}=1 \text {. }
$$

This proof, as well as that formerly given, is applicable to functions of any number of variables. For example, the equation

has the algebraic solution

$$
x^{4}+y^{4}+6 y^{3} z+7 x y z^{2}=1,
$$

$$
\begin{aligned}
& x=\frac{a}{\sqrt[4]{a^{4}+b^{4}+6 b^{3} c+7 a b c^{2}}}, \\
& y=\frac{b}{\sqrt[4]{a^{4}+b^{4}+6 b^{3} c+7 a b c^{2}}}, \\
& z=\frac{c}{\sqrt[4]{a^{4}+b^{4}+6 b^{3} c+7 a b c^{2}}} .
\end{aligned}
$$

And quite generally we can formulate as follows:-

If $\phi$ be a homogeneous function of the $\mathrm{nth}$ degree in $\mathrm{r}$ variables, the equation

has the algebraic solution

$$
\begin{aligned}
& \phi\left(x_{1}, x_{2}, x_{3}, \ldots, x_{r}\right)=1 \\
& x_{1}=\frac{a_{1}}{\sqrt[n]{\phi\left(a_{1}, a_{2}, \ldots, a_{r}\right)}}, \\
& x_{2}=\frac{a_{2}}{\sqrt[n]{\phi\left(a_{1}, a_{2}, \ldots, a_{r}\right)}},
\end{aligned}
$$

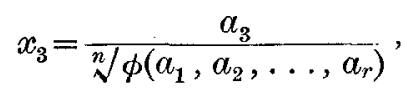

$$
\begin{aligned}
& \alpha_{r}=\frac{a_{r}}{\sqrt[n]{\phi\left(a_{1}, a_{2}, \ldots, a_{r}\right)}}
\end{aligned}
$$




\section{Solution of Equations consisting of two Homogeneous parts of Different}

Degrees. (Case II.)

These equations represent a class of central curves or surfaces essentially different from the preceding. In the case of equations of even degree the curve $\mathrm{F}_{n}(x, y)=\mathrm{A}^{n}$ cannot pass through the centre; while curves which are of the form $\mathrm{F}_{n}(x, y)=\mathrm{F}_{n-p}(x, y)$, when reduced to the lowest terms, generally pass through the centre, because their equations are satisfied by the values $x=0 ; y=0$.

Equations of the form $\mathrm{F}_{n}(x, y)=\mathrm{F}_{n-p}(x, y)$ are really honogeneous, or, at least, are reducible to homogeneous form by division.

Take, for example, the equation

By division we have

$$
\mathrm{A} x^{5}+\mathrm{E} x y^{4}=\mathrm{Q}\left(\mathrm{H} x^{3}+\mathrm{K} y^{3}\right) .
$$

$$
\frac{\mathrm{A} x^{5}+\mathrm{E} x y^{4}}{\mathrm{H} x^{3}+\mathrm{K} y^{3}}=\mathrm{Q}
$$

where the left-hand side is homogeneous, and of the 2nd degree. Consequently, by the results of the preceding analysis (sections 1 and 2), a solution is

$$
\left.\begin{array}{l}
x=a \cdot \sqrt{\frac{\mathrm{Q}\left(\mathrm{H} a^{3}+\mathrm{K} b^{3}\right)}{\mathrm{A} a^{5}+\mathrm{E} a b^{4}}} \\
y=b . \sqrt{\frac{\mathrm{Q}\left(\mathrm{H} a^{3}+\mathrm{K} b^{3}\right)}{\mathrm{A} a^{5}+\mathrm{E} a b^{4}}}
\end{array}\right\},
$$

where $a$ and $b$ are any quantities whatever. This may be directly verified as follows :-

Calling the expression under the root-sign $\omega$ and substituting, we have

and

$$
\begin{aligned}
\mathrm{A} x^{5}+\mathrm{E} x y^{4} & =\mathrm{A} a^{5}(\sqrt{ } \omega)^{5}+\mathrm{E} a b^{4}(\sqrt{ } \omega)^{5} \\
& =\left(\mathrm{A} a^{5}+\mathrm{E} a b^{4}\right)(\sqrt{ } \omega)^{5}
\end{aligned}
$$

$$
\begin{aligned}
\mathrm{Q}\left(\mathrm{H} x^{3}+\mathrm{K} y^{3}\right) & =\mathrm{Q}\left(\mathrm{H} a^{3}(\sqrt{ } \omega)^{3}+\mathrm{K} b^{3}(\sqrt{ } \omega)^{3}\right) \\
& =\mathrm{Q}\left(\mathrm{H} a^{3}+\mathrm{K} b^{3}\right)(\sqrt{ } \omega)^{3} .
\end{aligned}
$$

Hence

$$
\begin{aligned}
\frac{\mathrm{A} x^{5}+\mathrm{E} x y^{4}}{\mathrm{Q}\left(\mathrm{H} x^{3}+\mathrm{K} y^{3}\right)} & =\frac{\left(\mathrm{A} a^{5}+\mathrm{E} a b^{4}\right)(\sqrt{\omega})^{5}}{\mathrm{Q}\left(\mathrm{H} a^{3}+\mathrm{K} b^{3}\right)(\sqrt{\omega})^{3}} \\
& =\frac{\mathrm{A} a^{5}+\mathrm{E} a b^{4}}{\mathrm{Q}\left(\mathrm{H} a^{3}+\mathrm{K} b^{3}\right)} \cdot \omega \\
& =\frac{\mathrm{A} a^{5}+\mathrm{E} a b^{4}}{\mathrm{Q}\left(\mathrm{H} a^{3}+\mathrm{K} b^{3}\right)} \cdot \frac{\mathrm{Q}\left(\mathrm{H} a^{3}+\mathrm{K} b^{3}\right)}{\mathrm{A} a^{5}+\mathrm{E} a b^{4}} \\
& =1, \text { as it should be. }
\end{aligned}
$$

This quasi-extension of the original theorem may be formulated as follows :- 
If $\phi$ be a homogeneous function of the $n^{\text {th }}$ degree in $r$ variables, and $\psi$ a homogeneous function of the $(n-p)^{t h}$ degree in $r$ variables, the equation

has the solution

$$
\phi\left(x_{1}, x_{2}, \ldots, x_{r}\right)=\psi\left(x_{1}, x_{2}, \ldots, x_{r}\right)
$$

$$
\begin{aligned}
& x_{1}=a_{1} \sqrt[p]{\frac{\psi\left(a_{1}, \ldots, a_{r}\right)}{\phi\left(a_{1}, \ldots, a_{r}\right)}} \\
& x_{2}=a_{2} \sqrt[p]{\frac{\psi\left(a_{1}, \ldots, a_{r}\right)}{\phi\left(a, \ldots, a_{r}\right)}} \\
& x_{3}=\ldots \ldots \ldots \\
& \ldots \ldots \ldots \ldots
\end{aligned}
$$

The cases here examined evidently include the following forms :-

and

$$
\mathrm{F}_{n}(x, y)=\mathrm{A} x^{n-p} ; \mathrm{F}_{n}(x, y)=\mathrm{A} x^{n+p}
$$

$$
\mathrm{F}_{n}(x, y)=z^{p}
$$

where $z^{p}$ is a soluble function of other quantities, whose numerical value can be found and stated as a power of $z$.

4. Another mode of Solution; viz., by expressing each of the $\mathrm{r}$ Variables in terms of $\mathrm{r}-1$ New Variables.

(1) Where there are only two variables $\mathrm{X}$ and $\mathrm{Y}$, we have the relations $\mathrm{Y}=\mathrm{X} \tan \theta$; $\mathrm{X}=\mathrm{Y} \operatorname{cotan} \theta$, from which by substitution and division we may at once write the transformed equations of the homogeneous function $f(x, y)^{n}=1$,

$$
\begin{array}{cc}
1+a_{1} \tan \theta+a_{2} \tan ^{2} \theta \quad+\ldots=\frac{1}{\mathrm{X}^{n}} \\
\operatorname{cotan}^{n} \theta+a_{1} \operatorname{cotan}^{n-1} \theta+a_{2} \operatorname{cotan}^{n-2} \theta+\ldots=\frac{1}{\mathrm{Y}^{n}}
\end{array}
$$

Supposing a series of values of $X$ to be formed from (1), and tabulated for the argument $\theta$, then the column of values of $\mathrm{Y}$ is found by adding to each value of $\log \mathrm{X}$ the corresponding $\log \tan \theta$.

(2) When there are three or more variables $\alpha, \beta, \gamma$, \&c., they may, in like manner, be all expressed as functions of one of them, $a$, and new quantities. For this purpose, assume $\beta=a \tan \phi ; \gamma=a \tan \psi$, \&c., or more generally, $\beta=l \alpha ; \gamma=m \alpha ; \delta=n \alpha, \& c$.

Substituting these values, and dividing by $\alpha^{n}$, the transformed equation will then consist of a series of powers of $l, m, n$, \&c., equated to $1 / a^{n}$. Values of a may then le directly computed for any arguments or assumed values of $l, m, n$; and the other quantities, $\beta, \gamma, \delta, \& c$, are formed from (3).

The manner of doing this is shown by the following examples. (1.) Let the equation be

$$
x^{8}+x^{4} y^{2} z^{2}+3 x^{2} y^{2} z^{4}+y^{8}=w^{8}=1 \quad .
$$

$x$ is the quantity of which values are to be directly found ; $\theta$ is the angular coordinate in 
the plane $x y ; \phi$ is the angular coordinate in the plane $x z ; y=x \tan \theta ; z=x \tan \phi ;$ and the transformed equation is

$$
\begin{aligned}
& x^{8}\left\{1+\tan ^{2} \theta \cdot \tan ^{2} \phi+3 \tan ^{2} \theta \cdot \tan ^{4} \phi+\tan ^{8} \theta\right\}=1 \\
& \therefore \quad \frac{1}{x}=\left\{1+\tan ^{2} \theta \tan ^{2} \phi+3 \tan ^{2} \theta \tan ^{4} \phi+\tan ^{8} \theta\right\}^{1 / 8} .
\end{aligned}
$$

Suppose we want a series of values of $x$ and $y$ to the argument of $\tan \phi=1$ or $z=x$, the reduced equation is $1 / x=\left\{2+4 \tan ^{2} \theta\right\}^{1 / 8}$, from which the following values are directly

\begin{tabular}{|c|c|c|c|c|}
\hline$\theta={ }^{\circ}$. & $15^{\circ}$ & $30^{\circ}$ & $45^{\circ}$ & $60^{\circ}$ \\
\hline $\log \tan \theta$ & $=\overline{1} \cdot 4281$ & $\overrightarrow{1} \cdot 7614$ & 0.0000 & 0.2386 \\
\hline $\log \tan ^{2} \theta$ & $=\overline{\mathbf{2}} \cdot 8562$ & $\overline{1} \cdot 5228$ & 0.0000 & 0.4772 \\
\hline $\operatorname{Tan}^{2} \theta$ & $=0.0718$ & $0 \cdot 3333$ & 1.0000 & $3 \cdot 0000$ \\
\hline 4. $\tan ^{2} \theta+2$ & $=2 \cdot 2872$ & $3 \cdot 3332$ & $6 \cdot 0000$ & $14 \cdot 0000$ \\
\hline $\log \left(4 \tan ^{2} \theta+2\right)$ & $=0.3593$ & 0.5227 & $0 \cdot 7782$ & $1 \cdot 1461$ \\
\hline $1 / 8=\log 1 / x$ & $=0.0449$ & 0.0653 & 0.0973 & $0 \cdot 1433$ \\
\hline $\log x$ & $=\overline{\mathbf{1}} \cdot 9551$ & $\overline{1} \cdot 9347$ & $\overline{1} \cdot 9027$ & $\overline{1} \cdot 8567$ \\
\hline $\log y$ & $=\overline{1} 3832$ & $\overline{\mathbf{1}} \cdot 6961$ & $\overline{1} \cdot 9027$ & 0.0953 \\
\hline$x$ & $=0.9018$ & $0 \cdot 8604$ & 077993 & $0 \cdot 7190$ \\
\hline$y$ & $=0 \cdot 2416$ & $0 \cdot 7251$ & $0 \cdot 7993$ & $5 \cdot 2360$ \\
\hline
\end{tabular}
found :-

(2) Let the equation be

$$
\alpha^{10}+\beta^{4} \gamma^{3} \delta^{3}+\alpha^{4} \beta^{3} \gamma^{3}=1 .
$$

$\beta=l \dot{\alpha} ; \gamma=m a ; \delta=n \alpha ;$ and the transformed equation is

$$
1+l^{4} m^{3} n^{3}+l^{3} m^{3}=\frac{1}{a^{10}} .
$$

\begin{tabular}{|c|c|c|c|c|}
\hline$v$ & $20^{\circ}$ & $40^{\circ}$ & $60^{\circ}$ & $80^{\circ}$ \\
\hline $\log \tan ^{3} v$ & $=\overline{1} \cdot 5611$ & $\overline{\mathbf{1}} \cdot 9238$ & $0 \cdot 2386$ & 0.7537 \\
\hline $\log \tan ^{3} v$ & $=\overline{2} \cdot 6833$ & $\overline{\mathrm{I}} \cdot 7714$ & $0 \cdot 7158$ & $2 \cdot 2611$ \\
\hline $2+\tan ^{3} v$ & $=2 \cdot 0482$ & $2 \cdot 5907$ & $7 \cdot 1980$ & $184: 4500$ \\
\hline $\log \left(2+\tan ^{3} v\right)$ & $=0.3113$ & 0.4135 & 0.8572 & $2 \cdot 2658$ \\
\hline $1 / 10=\log 1 / \alpha$ & $=0.03113$ & 0.04135 & 0.08572 & 0.22658 \\
\hline $\log \alpha$ & $=\overline{\mathbf{1}} \cdot 96887$ & $\overline{\mathbf{1}} \cdot 95865$ & $\overline{1} \cdot 91428$ & $\overline{\mathbf{1}} \cdot 77342$ \\
\hline $\log \tan v$ & $=\overline{\mathrm{I}} \cdot 5611$ & $\underline{1} \cdot 9238$ & $0 \cdot 2386$ & $0 \cdot 7537$ \\
\hline $\log \delta$ & $=\overline{\mathbf{1}} \cdot 5300$ & $\overline{\mathbf{1}} \cdot 8825$ & $0 \cdot 1529$ & 0.5271 \\
\hline$\alpha$ & $=0.9309$ & 0.9093 & 0.8210 & 0.5934 \\
\hline$\delta$ & $=0.3388$ & 0.7630 & $1 \cdot 4220$ & $3 \cdot 3660$ \\
\hline
\end{tabular}

If it is desired to find values of $a$ and $\delta$ to the arguments $l=1, m=1$, the reduced equation is $2+n^{3}=1 / a_{10} \quad[n=\tan v$. $]$

An equation consisting of a single homogeneous part may also be reduced to polar coordinates and solved for $r$. If we write $r \cos \theta$ for $x$, and $r \sin \theta$ for $y$, and divide by $r^{n}$, the resulting equation is

$$
\operatorname{Cos}^{n} \theta+A_{1} \cdot \cos ^{n-1} \theta \cdot \sin \theta+A_{2} \cos ^{n-2} \theta \cdot \sin ^{2} \theta \pm \ldots \pm \sin ^{n} \theta=\frac{w}{r^{n}}=\frac{1}{r^{n}}
$$


whence $1 / r^{n}$ is found by summing the terms. But for purposes of computation the formulæ of the preceding paragraph are preferable, because they contain only half the number of trigonometrical quantities that are contained in the polar expression.

Where there are three variables, and it is desired to obtain values of a radius vector is terms of $\theta$ and the spherical angle $\phi$, the computation may also be simplified by making use of cylindro-polar coordinates. In this system $r$ is the radius vector in the plane of $X Y$; thence $x=r \cos \theta ; y=r \sin \theta ; z=r \tan \phi$. Each term of $x, y, z$ then contains at the most only three trigonometrical quantities to be computed, instead of five, as in the ordinary spherical system, and the angles $\theta$ and $\phi$ are the same.

The spherical radius vector, if required, can be afterwards found by the relation, spherical radius-vector $=r \sec \phi$. The equation of three variables transformed to cylindro-polar coordinates is of the form

$$
\begin{aligned}
& \cos ^{n} \theta+\left\{\mathbf{A}_{0} \cos ^{n-2} \theta \cdot \sin \theta+\mathbf{A}_{1} \cos ^{n-3} \theta \cdot \sin ^{2} \theta+\ldots\right\} \tan \phi+\left\{\cos ^{n-3} \theta \cdot \sin \theta+\ldots\right\} \tan ^{2} \phi \\
& +\ldots+\sin ^{n} \theta+\tan ^{n} \phi=1 / r^{n} .
\end{aligned}
$$

Examples of solutions effected by transformation to polar and cylindro-polar coordinates will be given in the sequel.

\section{Solution of certain Homogeneous Equations by the introduction of a New}

$$
\text { Variable. (Case III.) }
$$

It is only in the case of homogeneous equations that the $n^{\text {th }}$ root of the arbitrary term is a parameter or value of $x$ when $y=0$. In all other cases the parameter is determined by an equation in $x$ or $y$ (as the case may be), which in the case of the higher degrees can only be solved by approximation. Hence the method of homogeneous variation is not directly applicable. In applying the principle of homogeneous variation to functions which are not homogeneous, we must consider the function of two variables, as a particular value of a function of three variables in which $z$ has become unity. Thus, if we suppose a surface to be represented by an implicit homogeneous function $f(x, y, z)$, a plane section, parallel to the plane $x y$ and at a distance from the origin $z=1$, will be represented by the heterogeneous equation formed by the disappearance of the quantity $z$.

In order to solve an equation of the form $u_{n}+u_{n-1} \& c .=0$, we must first restore it to the homogeneous form by introducing such powers of $z$ as will make the equation homogeneous, and then endeavour to reduce $z$ to unity by homogeneous variation.

Consider the two following equations, in which the brackets include terms of the same degree in $x$ and $y:-$

$$
\begin{aligned}
& \left\{x+\mathrm{A}_{1} x^{n-1} y \pm \ldots \pm \mathrm{A}_{n} y^{n}\right\}+\left\{x^{n-1}+\mathrm{B}_{1} x^{n-2} y^{2} \pm \ldots \pm \mathrm{B}_{n-1} y^{n-1}\right\}+\left\{x^{n-2}+\& c .\right\}=0 . \\
& \left\{\xi^{n}+\mathrm{A}_{1} \xi^{n-1} \eta \pm \ldots \pm \mathrm{A}_{n} \eta^{n}\right\}+\left\{\xi^{n-1}+\mathrm{B}_{1} \xi^{n-2} \eta \pm \ldots \pm \mathrm{B}_{n-1} \eta^{n-1}\right\} \xi+\left\{\xi^{n-2}+\& c .\right\} \xi^{2}+\& c .=0
\end{aligned}
$$

The first form is a thoroughly heterogeneous equation, containing terms of degrees 
$n, n-1, n-2$, \&c. The second form is a homogeneous surface equation, from which (1) may be derived by giving to $z$ the value unity. Suppose $\xi$ and $\eta$ to be arbitrarily assumed quantities, and that we can by any known method find a value of the third coordinate $\zeta$, which will make the equation zero, then, dividing by $\zeta^{n}$, we eliminate $\zeta$. Thus, by division, the second of the above equations becomes

$$
\left\{\left(\frac{\xi}{\zeta}\right)^{n}+\mathrm{A}_{1}\left(\frac{\xi}{\xi}\right)^{n-1}\left(\frac{\eta}{\xi}\right) \mp \ldots \mp\left(\frac{\eta}{\xi}\right)^{n}\right\}+\left\{\left(\frac{\xi}{\xi}\right)^{n-1}+\mathrm{B}_{1}\left(\frac{\xi}{\xi}\right)^{n-2}\left(\frac{\eta}{\xi}\right) \mp \ldots \mp\right\} \frac{\xi}{\xi}+\left\{\left(\frac{\xi}{\xi}\right)^{n-2}+\left(\frac{\xi}{\xi}\right)^{n-3}\left(\frac{\eta}{\xi}\right) \mp \ldots \mp\right\} \frac{\xi^{2}}{\xi^{2}}=0
$$

The quantities outside the brackets are unity, and the quantities inside the brackets constitute a solution or value of equation (1), where $\xi / \zeta$ is a value of $x$, and $\eta / \zeta$ is a value of $y$, while $z$ as a separate quantity has disappeared. $\zeta$ may be considered either as a third dimension or as a variable parameter.

Accordingly if $\zeta$ can be found and the arbitrary equation formed, the solution is at once obtained by dividing $\xi$ and $\eta$ respectively by $\zeta$. Let $\mathrm{S}_{n}$ represent the numerical value of the homogeneous part within the first bracket formed by assuming arbitrary quantities $\xi, \eta ; \mathbf{S}_{u-1}$ is the numerical value of the terms within the second bracket (which are all of the degree $n-1$ ), and so on, and the equation is

$$
\mathrm{S}_{n}+\mathrm{S}_{n-1} \xi+\mathrm{S}_{n-2} \xi^{2}+\mathrm{S}_{n-3} \xi^{3}+\mathrm{S}_{n-4} \xi^{4}+\& \mathrm{c} .=0
$$

It is easily seen that the possibility of solution does not at all depend on the degree of the given equation, but upon the relative degrees of the terms of $\zeta$, which it is necessary to introduce. If the equation consists of only two homogeneous parts, suppose of the 9th and 3rd degrees, we have a simple equation to determinate $\zeta$, as in this example $x^{9}+\mathrm{A}_{1} x^{8} y+\ldots=x^{3}+\mathrm{B}_{1} x^{2} y+\ldots \& c_{\text {. }}$, which may be written $u_{9}=u_{3}$. By introducing the quantity $z=1$ this becomes

$$
\begin{gathered}
\left\{x^{9}+\mathrm{A}_{1} x^{8} y+\ldots\right\}=\left\{x^{3}+\mathrm{B}_{1} x^{2} y+\ldots\right\} z^{5} \\
\xi=\frac{\left\{\xi^{9}+\mathrm{A}_{1} \xi^{8} \eta+\& \mathrm{c} .\right\}^{1 / 5}}{\left\{\xi^{3}+\mathrm{B}_{1} \xi^{2} \eta+\& \mathrm{c} .\right\}^{1 / 5}}
\end{gathered}
$$

Then by the introductory theorem we find

$$
x_{1}=\xi / \xi ; y_{1}=\eta / \xi
$$

This is the case already considered in section 3. Similarly, if the auxiliary equation in $\xi, \eta$, and $\zeta$ contains only the first and second powers of $\zeta$, we have a quadratic equation between $\zeta$ and the sums of the numerical terms of the assumed quantities, whence $\zeta$ may be found, and thence exact solutions of $x$ and $y$. If the auxiliary equation contains $\zeta^{2}$ and $\zeta^{4}$ or $\zeta^{3}$ and $\zeta^{6}$, we have a quadratic equation to determine $\zeta^{2}$ or $\zeta^{3}$, whose root may then be extracted. Or, finally, there may be a soluble cubic or biquadratic equation in $\zeta$ or some power of $\zeta$.

If the equation contains an arbitrary term, this is equivalent to an additional 
homogeneous part. The arbitrary term may either be treated as a coefficient or reduced to unity by division, and in the auxiliary equation it is replaced by $\zeta^{n}$ in order to form a homogeneous function equated to zero.

The method of this section is essentially the same as is implied in the following :-

A solution of the equation

is

$$
x^{5}+6 x^{4} y+3 x^{4}+5 x y^{3}+2 x^{3}+4 y^{3}=0
$$

$$
\begin{aligned}
& x=\frac{a}{\mathrm{R}} \\
& y=\frac{b}{\mathrm{R}},
\end{aligned}
$$

where $R$ is a root of the equation

$$
\left(a^{5}+6 a^{4} b\right)+\left(3 a^{4}+5 a b^{3}\right) \mathrm{R}+\left(2 a^{3}+4 b^{3}\right) \mathrm{R}^{2}=0 .
$$

For on substituting we have the left-hand side

$$
\begin{aligned}
& =\frac{a^{5}}{\mathrm{R}^{5}}+\frac{6 a^{4} b}{\mathrm{R}^{5}}+\frac{3 a^{4}}{\mathrm{R}^{4}}+\frac{5 a b^{3}}{\mathrm{R}^{4}}+\frac{2 a^{3}}{\mathrm{R}^{3}}+\frac{4 b^{3}}{\mathrm{R}^{3}} \\
& =\frac{a^{5}+6 a^{4} b+\left(3 a^{4}+5 a b^{3}\right) \mathrm{R}+\left(2 a^{3}+4 b^{3}\right) \mathrm{R}^{2}}{\mathrm{R}^{5}} \\
& =\frac{0}{\mathrm{R}^{5}} \\
& =0, \text { as it should be. }
\end{aligned}
$$

And generally-

If $\phi_{n}(x, y)$ denote a homogeneous equation of the $n^{\text {th }}$ degree, the equation

has for solution

$$
\phi_{n}(x, y)+\phi_{n-1}(x, y)+\ldots+\phi_{1}(x, y)+\phi_{0}(x, y)=0
$$

$$
\begin{aligned}
& x=\frac{a}{\mathrm{R}} \\
& y=\frac{b}{\mathrm{R}},
\end{aligned}
$$

where $R$ is a root of the equation

$$
\phi_{n}(a, b)+\mathrm{R} \phi_{n-1}(a, b)+\mathrm{R}^{2} \phi_{n-2}(a, b)+\ldots+\mathrm{R}^{n-1} \phi_{1}(a, b)+\mathrm{R}^{n} \phi_{0}(a, b)=0
$$

In the case of a heterogeneous function of three variables, there is a choice of four solutions. (1) A quantity $\mathrm{P}$ may be introduced which will make the equation homogeneous as a whole; and the equation may then be solved for $\mathrm{P}$ by treating the arbitrarily assumed quantities, $\xi, \eta, \zeta$, as known quantities. Then, dividing by $\mathrm{P}^{n}$, values of $x, y, z$ are found for $\mathrm{P}=1$. (2,3, and 4) Any one of the quantities (say $z$ ) may be assumed as constant during the operation, or $z=\alpha$ and a quantity $\mathrm{P}$ is then to be intro- 
duced which will make the equation a homogeneous function of $\mathrm{P}$ and the other two quantities, $x$ and $y$. Then dividing by $\mathrm{P}^{n}, x$ and $y$ are found for $z=a ; \mathrm{P}=1$. In this way a series of values of $x$ and $y$ may be found to any argument $z$. The choice of the method will of course be determined by the possibility of solving the equation in P. Unless the degree of the equation is very high, or the terms very numerous, it will generally be found that an equation can be formed from which $\mathrm{P}$ may be determined, and the corresponding values of $x, y$, and $z$ deduced by homogeneous variation.

As in the case of plane curves, we see that in the case of surfaces also, any surface may be expressed as a homogeneous equation of the three variables and the intercept $\mathrm{P}$ on one of the axes. Also, any function of identical form, with a different value of $P$, is a similar surface.

The method is evidently capable of extension to equations of any number of unknown quantities.

There are two distinct geometrical interpretations of the processes here given, according as we consider the new quantity $z$ as being in a different plane from $x, y$, or in the same plane.

(1) In the former case $z$ is a third coordinate, and the 3-dimensional homogeneous equation $f(x, y, z)=0$ always and necessarily represents a conical surface. This may be proved (without drawing on the methods of the differential calculus) by transforming the equation to cylindrical coordinates. $\mathrm{XY}$ is the reference plane in which $r$ and $\theta$ are measured; $z$ is then perpendicular to that plane. Then writing $r \cos \theta$ for $x, r \sin \theta$ for $y$, we have a homogeneous equation in $r$ and $z$ with trigonometrical coefficients. Accordingly if $r$ and $z$ be varied, while $\theta$ remains unchanged, we have by the introductory proposition $r_{1} / z_{1}=r_{2} / z_{2}=r_{3} / z_{3}$, \&c. This can only be true if $r$ and $z$ are coordinates of the same generating line, which of course lies in a plane passing through the axis of $z$ and making the angle $\theta$ with the plane XZ. More simply, as the result of the transformation to cylindrical coordinates is to form a homogeneous function, $f(r, z)=0$, this is known to be the equation of two right lines, and the surface is then shown to be made up of generating lines passing through the origin, which is the definition of a conical surface.

In order that the equation $f(x, y, z)=0$ may have real solutions, the highest power of one of the quantities must be negative ; and it is easy to see that the homogeneous function of the $n^{\text {th }}$ degree, $f(x, y, z)=0$ is the asymptotic cone of all the concentric and similar surfaces which can be found by equating the same function to an arbitrary term $\mathrm{P}^{n}$. It is in fact the limiting form of this series of concentric and similar surfaces when the parameter $\mathrm{P}$ vanishes.

(2) I began by observing that we might conceive the quantity $z$ (which was introduced for the purpose of rendering the equation to be solved homogeneous) as being either in a different plane from $x$ and $y$, or in the same plane. If it is considered as being in the same plane, it is the parameter of the non-homogeneous curve, and may be denoted by $\mathrm{P}$. The proof is as follows:-Compare the two subjoined equations, in which 
the original heterogeneous equations $x y, \xi \eta$, have been made homogeneous by introducing supplementary powers of $\mathrm{P}$ and $\Pi$,

$$
\begin{aligned}
& \left\{x^{n}+\mathrm{A}_{1} x^{n-1} y \mp \mp y^{n}\right\}+\left\{\mathrm{B}_{1} x^{n-1} \mathrm{P}+\mathrm{B}_{1} x^{n-2} y \mathrm{P} \mp \mp \mathrm{B}_{n} y^{n-1} \mathrm{P}\right\}+\left\{\mathrm{C}_{1} x^{n-2} \mathrm{P}^{2}+\mathrm{C}_{1} x^{n-3} y \mathrm{P}^{2} \mp \mp y^{n-2}\right\}=0 \\
& \left\{\xi^{n-1}+\mathrm{A}_{1} \xi^{n-1} \eta \mp \mp \eta^{n}\right\}+\left\{\mathrm{B} \xi^{n-1} \Pi+\mathrm{B}_{1} \xi^{n-2} \eta \Pi \mp \mp \mathrm{B}_{n} \eta^{n-1} \Pi I\right\}+\left\{\mathrm{C}^{n-2} \Pi^{2}+\mathrm{C}^{n-3} \eta \Pi^{2} \mp \mp \eta^{n-2}\right\}=0 .
\end{aligned}
$$

As the equations are homogeneous and identical in form, they represent similar curves; and according to the fundamental theorem of this paper the one form may be derived from the other by multiplying every term by a constant, that is by $(\mathrm{P} / \Pi)^{6}$. Hence $x, y$, and $\mathrm{P}$ are obtained from $\xi, \eta$, and $\Pi$ by multiplying each by the factor $\mathrm{P} / \Pi$. This can only be true if $P$ and $\Pi$ are the parameters or the same multiple of parameters of the respective curves.

It may occur as a difficulty that in the case of heterogeneous curves, the quantity $\mathbf{P}$ does not correspond to the value $x_{0}$ of $x$ when $y$ is equated to zero. But $\mathrm{P}$ can easily be shown to be proportional to $x_{0}$. For suppose $y$ and $\eta$ in the two curves of the example equated to zero, the equations are then of the form

$$
\begin{aligned}
& x_{0}^{n}+\mathrm{B} x_{0}^{n-1} \mathrm{P}+\mathrm{C} x_{0}^{n-2} \mathrm{P}^{2} \mp \mp=\mathrm{P}^{n} \\
& \xi_{0}+\mathrm{B} \xi_{0}^{n-1} \mathrm{I}+\mathrm{C} \xi_{0}^{n-2} \Pi^{2} \mp \mp=\Pi^{n} .
\end{aligned}
$$

Dividing by the highest powers of $P$ and $\Pi$, we have

$$
\begin{aligned}
& \left(\frac{x_{0}}{\mathrm{P}}\right)^{n}+\mathrm{B}\left(\frac{x_{0}}{\mathrm{P}}\right)^{n-1}+\mathrm{C}\left(\frac{x_{0}}{\mathrm{P}}\right)^{n-2} \mp \mp=1 \\
& \left(\frac{\xi_{0}}{I I}\right)^{n}+\mathrm{B}\left(\frac{\xi_{0}}{\bar{I}}\right)^{n-1}+\mathrm{C}\left(\frac{\xi_{0}}{\bar{I}}\right)^{n-2} \mp \mp=1 .
\end{aligned}
$$

Hence by the known law of expansions, $x_{0} / \mathrm{P}=\xi_{0} / \Pi$, or the quantities $\mathrm{P}$ and $\Pi$ of similar curves have a constant ratio to the intercepts $x_{0} \xi_{0}$. They are therefore virtual parameters.

(3) The case of a homogeneous equation of the $n^{\text {th }}$ degree equated to a term $Z^{n}$ or $\mathrm{P}^{n}$, with which the paper commences, is now seen to be merely an explicit form of the general conical or parametral equation, $f(x, y, z)=0$. If the explicit term be considered as a third coordinate $(z)$, the conical surface is referred to a plane of symmetry, $x y$, and an axis of symmetry $z$. In the implicit function the projections of the similar parallel sections in the plane $x y$ are neither similar nor symmetrical; and the similar sections are only found by taking $z$ into account.

So with the implicit function considered as of three quantities in one plane. The parameter, $\mathrm{P}$, is evidently not the principal parameter of the curve, but is the value of the intercept on the axis of $x$ in the system of axes proposed.

From this investigation we see that any plane curve whatever may be expressed as a homogeneous function of rectangular coordinates and the intercept on one of the axes. When so expressed, it is a similar and similarly situated curve with respect to any other curve expressed as the same function with a different value of $P$. 


\section{Soluble Cases of the Homogeneous Function $f(x, y, z)^{n}=0$.}

This function, as has been observed, represents a conical surface, being the asymptotic cone of all the concentric and similar surfaces that can be formed by equating the same function of $x, y, z$, to different arbitrary terms.

Unless the equation contains a large number of terms, it can in general be solved by taking arbitrary values of those two quantities which are most involved, and solving for the one which is least involved.

These solutions represent points on the conical surface, and if it is desired to obtain such solutions in series, so as to represent a plane section or curve, they may be reduced by division to the argument $x=1, y=1$, or $z=1$ as desired. It is only necessary to tabulate one such series; because the surface is conical, and values of $y$ and $z$ may be obtained to any other argument $x=\alpha$, by merely multiplying the tabular values of $y$ and $z$ by $a$. Consider, for example, the equation of a homogeneous surface of this form,

$$
x^{8}+\mathrm{A} x^{6} y^{2}+\mathrm{B} x^{5} z^{3}+\mathrm{C} x^{2} z^{6}+\mathrm{C} y^{2} z^{6}+\mathrm{B} y^{5} z^{3}+\mathrm{A} x^{2} y^{6}+y^{8}=0 \text {. }
$$

Here the equation is symmetrical in $x$ and $y$, but contains no powers of $z$ except the 6th and the 3rd. Accordingly, we may form an equation by assuming values $x_{1}$ and $y_{1}$, and then solving the quadratic equation in $z^{3}$, the root of which, being extracted, is a solution. That is to say, the values $x_{1}, y_{1}$, and $z_{1}$ thus found satisfy the equation.

\section{Solution of Homogeneous Equations of Functions of the Variables. (Case IV.)}

Assume

$$
v_{1}=a x^{p}+b y^{q}+c ; v_{2}=d x^{p}+e y^{q}+f ; v_{3}=\ldots
$$

Any homogeneous expression in $v_{1} v_{2} \ldots$ equated to an arbitrary term, or to another homogeneous expression in $v_{1} v_{2} \ldots$ of a different degree, can be solved by the methods previously given. Values of $v_{1} v_{2} \ldots$ being first found, we have then two simple equations for determining $x^{p}$ and $y^{q}$ in terms of these values, whence $x$ and $y$ are found.

The original equation is of course heterogeneous when the quantities $\alpha x^{p}+b y^{q}+c, \& c$, are substituted in place of $v, \& c$; and by means of this new application of the fundamental theorem, an endless variety of heterogeneous equations may be formed and solutions in series obtained. It is evidently a condition of the possibility of solving such equations that the number of factors $v_{1} v_{2}$, \&c., shall not exceed the number of constituent quantities, $x, y$, \&c., of which values are to be found.

If the indices $p, q, \& c$, are even, the curve or surface is central; but the converse does not necessarily hold. Thus $v_{1}=a x+b ; v_{2}=c y+d$, gives a central curve from an excentric origin, of which PI. VI. figs. 5 and 6 are illustrations. If one of the quantities, $v_{1}$, be taken $=\alpha+\sqrt{x}$ the curve will only have single symmetry.

If we take $v_{1}^{2}=x^{2}+z^{2} ; v_{2}^{2}=y^{2}+z^{2}$, thus a series of values of $x$ and $y$ may be found to an invariable value of $z$, and the series of points so determined will trace out one or 
more contour lines of the homogeneous surface in $x, y, z$, which is represented by the equation. Examples of these are given in the sequel.

Other applications of the combination of soluble functions of $x$ and $y$, or $r$ and $\theta$, will readily suggest themselves. The following may suffice as illustrations :-

(1) Let the equation consist of powers of quantities $\left(x^{2}+y^{2}\right)$ and $\left(x^{2}-y^{2}\right)$ as

$$
\left(x^{2}+y^{2}\right)^{n}+\mathrm{A}_{1}\left(x^{2}+y^{2}\right)^{n-1}+\mathrm{A}_{2}\left(x^{2}+y^{2}\right)^{n-2}++\ldots=\mathrm{A}_{n}\left(x^{2}-y^{2}\right)^{p} .
$$

This is equivalent to

$$
r^{2}+\mathrm{A}_{1} r^{2 n-2}+\mathrm{A}_{2} r^{2 n-4}++\ldots=r^{2 p} \cos ^{p} 2 \theta \cdot \mathrm{A}_{n},
$$

whereby $\theta$ is determined in terms of $r$, and thence $x$ and $y$.

$$
\left(x^{l}+y^{m}\right)^{n}+\mathrm{A}_{1}\left(x^{l}+y^{m}\right)^{n-1}+\mathrm{A}_{2}\left(x^{l}+y^{m}\right)^{n-2}++=\mathrm{A}_{n}\left(x^{l}-y^{m}\right)^{p} .
$$

Take $u^{2}=x^{l} ; v^{2}=y^{m}$; and solve the resulting equation in $u$ and $v$, which is of the above form. Then $u$ and $v$ are found from $r$ and $\theta$, whence $x$ and $y$ are determined.

(3) This solution is evidently capable of extension to any function in the form on the left side of the sign of equality, where the quantity on the right side can be expressed as the power of a cosine or sine of a multiple of $\theta$, or a soluble function of such a sine or cosine. In these equations each term is a homogeneous function, and the solution depends partly on this circumstance.

(4) If the right-hand term consists of a power of $x$ alone or of $y$ alone, the equation is solved by writing $r^{p} \cos ^{p} \theta$ for $x^{p}$, or $r^{p} \sin ^{p} \theta$ for $y^{p}$.

(5) If the left side of the equation consists of powers of the quantity $\left(x^{l}-y^{m}\right)$, and the right side of a single term $x^{p}$ or $y^{q}$, or $\left(x^{l}+y^{m}\right)^{p}$ solutions of $r$ can be found to the argument $\theta$, and thence $u_{1} v_{2}$ and $x y$ are similarly found.

\section{To find the Condition under which parallel Sections of a given Surface may be similar Curves.}

The condition is evidently fulfilled if one of the quantities $x, y, z$ be given explicitly in the equation. The surface may be conceived as traced by the motion of a generating curve controlled by a guiding curve.

Suppose the generating curve to be a homogeneous function of $x, y$, equated to a function of $z$ only. Then, as the sections parallel to XY are to be similar curves, the generating curve must move parallel to itself with varying parameter, and so as always to touch a guiding curve in the place XZ. Let the equation of the guiding curve be of the form

$$
x^{n}-z^{n} \pm \mathrm{B}_{1} z^{n \pm 1} \pm \mathrm{B}_{2} z^{n \pm 2} \pm \ldots+\mathrm{B}_{n} z^{n \pm p}=0 .
$$

Then the equations of the generating and guiding curves are

$$
\begin{aligned}
& f(x y)=x^{n}+\mathrm{A}_{1} x^{n-1} y+\mathrm{A}_{2} x^{n-2} y^{2} \mp \mp \mathrm{A}_{n} y^{n}=p \\
& f\left(x^{\prime} y^{\prime}\right)=x^{\prime n}-z^{\prime n} \pm \mathrm{B}_{1} z^{h_{ \pm 1}} \pm \ldots+\mathrm{B}_{n} z^{n_{ \pm}}=0
\end{aligned}
$$


In equations (1) and (2) $p$ is the variable parameter of the section parallel to $x y$, and is evidently the ordinate in the principal plane $x z$. Hence, $x=p ; x^{\prime n}=p^{n}=f(x y)$.

Also the value of $z$ in the two sections is the same.

Substituting in (2) the value of $x^{\prime}$ above found, we have for the equation of the surface

$$
x^{n}+\mathrm{A}_{1} x^{n-1} y+\mathrm{A}_{2} x^{n-2} y^{2} \mp \mp \mathrm{A}_{n} y^{n}=z^{n} \mp \mathrm{B}_{1} z^{n-1} \pm \ldots+\mathrm{B}_{n} z^{n \pm p}
$$

whence values of $x$ and $y$ can be found by homogeneous variation to any argument $z$. For, we have only to suppose $z$ constant, and to state the sum of the terms of $z$ as an arbitrary quantity in the form $\mathrm{P}^{n}$, and the problem is reduced to that of the introductory proposition.

In the example given, the plane XY is a plane of symmetry, and the axis $z$ is an axis of symmetry; but these considerations are insufficient to determine similar parallel sections unless the quantities $x$ and $y$ are combined homogeneously. It is easy to see that in the example given the sections parallel to $\mathrm{XZ}$ and $\mathrm{YZ}$ are not similar, because they are neither homogeneous functions of the respective pairs of variables, nor of these quantities and the parameter.

On trial it will be found that no other generating curve, except a homogeneous curve, gives similar parallel sections. If possible, let the sections of the unknown surface taken parallel to the following symmetrical equation be similar curves

$$
x^{n}+\mathrm{A}_{1} x^{n-2} y^{2}+\mathrm{A}_{2} x^{n-4} y^{4} \mp \mp \mathrm{A}_{2} x^{4} y^{n-4}+\mathrm{A}_{1} x^{2} y^{n-2}+y^{n}=\mathrm{P}^{n} \text {. }
$$

In order that the sections parallel to the plane of $x y$ may be similar, their equations must be homogeneous functions of $x, y$ and $\mathrm{P}$, as has been proved. Hence the condition is satisfied by substituting $z=\mathrm{P}$ or $f(z)=\mathrm{P}$.

It thus appears that a central surface, other than a conical surface, will not furnish sections parallel to $x y$ which are similar curves, unless the third quantity, $z$, be given explicitly, so that the terms of $x$ and $y$ alone constitute a homogeneous equation. In treating of equations in this perfectly general form, one is apprehensive of some possible exception or flaw in the demonstration; I have accordingly taken pains to verify this conclusion, by endeavouring to find values of $x$ and $y$ from the surfaces generated by various non-homogeneous curves, choosing the most symmetrical forms referred to conjugate axes as being those which were most likely to give results.

In every instance the values of $x$ and $y$, found on the assumption that the parallel sections were similar, failed to satisfy the original equation, although they must necessarily have done so had the hypothesis been correct.

I consider it then demonstrable that the sections of homogeneous surfaces are only similar curves when the sections are homogeneous functions of $x$ and $y$ equated to powers of $z$ uncombined with $x$ or $y$. In other words, the homogeneous function must be of the form $f(x, y)=f(z, \mathrm{P})$; otherwise parallel sections will be dissimilar. 


\section{Classification and Forms of Curves considered as Sections of Surfaces whose Equations are Homogeneous.}

In this chapter I do not enter on the question of the singularities of curves, a theme which has already been the subject of much learned investigation. My purpose is (1) to discover the different elementary and symmetrical forms of the curves of a given degree, which may be considered as the sections of a homogeneous surface parallel to its principal planes; and (2) from these elementary forms to show how by variation of the unknown quantities corresponding types of unsymmetrical curves of the $n^{\text {th }}$ or given degree may be obtained, and the surfaces traced in series of contour-lines.

I ought here to point out that the motive of this investigation is somewhat different from that of Mr Frost's valuable work on Curve Tracing.

In a treatise on Curve-Tracing in general, the exact determination of the locus of the curve is of course unattainable, and only approximate methods are used.

In the present paper, only those curves are considered whose loci can be exactly traced, by solving these equations rigorously for successive positions of $x$ and $y$. In the diagrams, which are photographic reductions of the original tracings on diagram-paper, the error at any point ought not to exceed $\frac{1}{500}$ of an inch.

It is perhaps unnecessary to prove that every homogeneous equation of three variables represents a surface symmetrical about three principal axes of symmetry, which it is convenient to consider as placed perpendicularly to one another. This follows from the consideration that when the homogeneous equation is transformed to polar coordinates, it contains only the highest power of $r$, which in the case of a curre of even degree has always equal positive and negative roots." In the case of curves of uneven degree, the same results are obtained by considering the sign of the arbitrary term indeterminate, - as it evidently ought to be, because by so treating it, we obtain from the equations of uneven degrees forms which are strictly analogous to those of the nearest even degrees.

This being premised, if in the surface represented by the given homogeneous equation we take for the axis of $Z$, the direction of the radius vector of maximum length; and if the surface be referred to this axis $Z$ and to a central plane perpendicular to $Z$ in which angles are denoted by $\theta$, then for every value of $\theta$ and $\phi$ there are four equal values of $r$ corresponding to the four permutations of the positive and negative values of $\theta$ and $\phi$ and also other four formed from $\pi-\theta$ and $\phi$. Hence, for any plane through the axis of $Z$, there are four equal values of $r$, and the curve is symmetrical about $Z$ and the diameter in the plane $X Y$. By transforming to an axis $X$ coinciding with the maximum radius vector of the central plane and a plane perpendicular to it, similar conclusions are obtained for all diametral sections through $X$, and also for all diametral sections through $\mathrm{Y}$, the line of intersection of the first and second reference planes. Thus the symmetry of the surface with reference to three principal planes and their intersections is established. 
A homogeneous surface may have more than one set of axes of symmetry. Some of these may be conjugate diameters meeting the surface at finite points, and some of them may be asymptotic lines.

In a central equation the axes of reference are asymptotic lines, if the equation wants the highest powers of the three variables; because then, dividing the equation by the lowest power of any of the variables, suppose $x^{p}$, we find for the values, $y=0, x=0$, the corresponding value $z=\mathrm{A} / x^{p}=\infty$.

When a homogeneous central surface is referred to axes of symmetry, its equation must consist either entirely of terms of even powers of each variable $z, x$ or $y$, or entirely of terms of uneven powers of the same variable; because then only will the value of the term be unaltered when $x$ is changed to $-x$, or $y$ is changed to $-y$. It is of course only in curves of even degree that $x$ and $y$ are both even or both uneven, and therefore curves of uneven degree have in general only single symmetry unless the sign of the arbitrary term is treated as indeterminate. Accordingly,

1. If the equation of a homogeneous surface is of uneven degree, and consists of terms of even powers of $x$ and uneven powers of $y$, the axes of reference are asymptotic lines.

2. If the homogeneous surface equation, being of uneven degree, consists of terms of uneven powers of the variables, the axes of reference coincide with finite conjugate diameters; but this condition can only be fulfilled if the equation is of the form-

$$
x^{n} \mp y^{n} \mp z^{n}=\mp A^{n},
$$

where the sign $\mathrm{A}^{n}$ is indeterminate.

3. Again, if the equation of a homogeneous surface be of even degree, and consists entirely of terms of uneven powers of the variables, the axes of reference are asymptotic lines.

4. If the equation being of even degree consists of terms of even powers of the variables, the axes of reference coincide with finite conjugate diameters, unless the highest powers of the variables are awanting.

5. If, in any of these cases, the surface is expressed by a symmetrical equation,that is, if the equation consists of pairs of homologous terms, the signs in all the pairs being either like or unlike,- - the three axes are equal; and the surface is also symmetrical about six secondary conjugate axes, which bisect the angles between each pair of the first set. Moreover, there are two planes through the axis of $Z$ and a secondary axis lying between the axes $\mathrm{X}$ and $\mathrm{Y}$, which are planes of symmetry; and two for each similar combination; that is, six planes of symmetry in addition to those originally given.

6. If the equation be of the form $f^{n}\left(x / a, y^{/} b, z / c\right)=1$, and be a symmetrical function of these ratios, the surface will of course be a "3-dimensional projection," or homogeneous transformation of the corresponding function of $x, y, z$. It is evident from known principles that all lines and planes of rectangular symmetry will be projected into lines and planes of oblique symmetry; and the secondary planes and lines will bisect 
those parallel to conjugate planes and lines, but will not bisect the angles between the principal diameters and principal planes.

7. Paragraphs 1,2, 3, and 4 may be applied to plane curves by suppressing the element $Z ; 6$ also applies to plane curves, and it will be shown that the inclination of the secondary axis, $x$, to the principal axis, $\mathrm{X}$, is given by the relation, $\tan \theta=b / a$. In the further discussion of the subject I shall use the term "Diametral Equation" to express the equation of a curve when referred to axes of symmetry. If an equation containing only even powers of the variables $x$ and $y$ be also a symmetrical expression, the curve has fourfold symmetry, because the symmetrical form of the equation shows that the axes of reference are equally inclined to a second pair of conjugate axes. There are then eight points at which the value of $R$ is either a maximum or a minimum. This is a property which is not lost by projection. A Symmetrical Diametral Equation is an equation which is itself symmetrical; where therefore the curve is equiaxial and has fourfold symmetry.

\section{Classification of Central Curves of the Form $\mathrm{F}(x, y)^{n}=\mathrm{A}^{n}$.}

A central function of two variables equated to an arbitary term may be either homogeneous or heterogeneous. In the first case, the equation may represent either a central section of the general homogeneous surface, or a section taken parallel to a principal plane of any homogeneous surface whose equation contains only the highest power of Z. In the second case, the equation represents a section taken parallel to a principal plane of the general homogeneous surface. Reference is made on this point to the preceding part of the paper.

If we begin by considering homogeneous symmetrical forms, or forms which are the projections of these, it is evident that the equations must be composed by the multiplication of factors of the forms,

$$
\left(\frac{x}{a} \pm \frac{y}{b}\right)^{p} ;\left(\frac{x^{2}}{a^{2}} \pm \frac{y^{2}}{b^{2}}\right)^{q} ; \ldots\left(\frac{x^{n}}{a^{n}} \pm \frac{y^{n}}{b^{n}}\right)^{r} .
$$

The number of possible symmetrical equations is, however, very much less than the possible permutations of such factors; and it is not difficult to see that the required number is that of the permutations of the positive and negative signs in a symmetrical equation containing only even, or only uneven powers of $x$ or $y$. From the preceding remarks it is seen that a diametral symmetrical equation represents a curve which has two pairs of conjugate axes, each axis bisecting the angles between the axes of the other pair; and that such axes are either asymptotes or finite conjugate diameters.

(1) Oval Forms.-If the given equation is homogeneous, and if the four axes of symmetry coincide with finite diameters, the equiaxial curve is generally a symmetrical oval entirely concave to the centre. In this species, if the original equation consists of terms of even powers, the equation of the curve when transformed to secondary axes as 
axes of reference, consists also of even powers of the variables. In this type of curve, when referred to either pair of conjugate axes, all the terms of the variables are positive. It will presently be seen that where some of the homologous pairs of terms are positive and some are negative, the curve may be an inflexional oval of double symmetry, passing into an inflexional hyperbolic for certain values of the coefficients.

(2) Hyperbolic Forms. - If the axes consist of a pair of finite diameters and a pair of asymptotes, the curve consists of two or more infinite branches symmetrically placed, which may be either all equal or of two sets. These may be termed continuous or discontinuous hyperbolics, according as the branches are all real, or consist of real and imaginary (or conjugate) branches in alternate order. If in each pair of homologous terms the signs are unlike, the branches are entirely convex to the centre, the inflexional hyperbolic being represented by an equation of pairs of homologous terms, some of which pairs are positive and some negative, or of unlike signs.

All equiaxial curves, whether of the first or the second class, complete their phases within a quadrant. In curves of the second class, the secondary axes, although asymptotic, are true diameters, because the form of the equation shows that each asymptote bisects all ordinates drawn parallel to the other; that is, it bisects the intercepts made by two adjacent branches, which may be either both real, or one of them real and the other conjugate.

(3) Projections of Equiaxial Forms.-By writing $x / \alpha$ for $x$ and $y / b$ for $y$ in any homogeneous equiaxial equation, the equation of the projection of the equiaxial curve is formed. The curves of the series which may be formed by projection have the same general resemblance to the primitive equiaxial forms that ellipses and common hyperbolas have to the primitive forms of the circle and the equilateral hyperbola.

(4) Heterogeneous Central Equations.-Every equation of even degree, and containing only even powers of the variables (although not homogeneous), represents a central curve; and if the equation be a symmetrical expression, the curve is equiaxial. I shall here only consider those heterogeneous central forms which represent sections of the symmetrical homogeneous surface equation.

It has been pointed out that every heterogeneous central expression represents a section of a homogeneous and central surface taken parallel to a principal plane. There is then no specific difference between homogeneous and heterogeneous central curves pertaining to the same surface. The highest homogeneous part of the equation is the limiting equatorial section, where the terms compounded with $Z$ disappear, and the general form of the curve depends solely on the highest homogeneous part of its equation.

(5) With regard to those curves whose equations are not symmetrical functions of $x$ and $y$, or $x / a$ and $y / b$, it is in general not possible to find secondary axes to these. But the curves of unsymmetrical expression are assimilated to those whose equations are symmetrical by the Rule of Signs, which will presently be deduced, and their traces, computed by the homogeneous method, prove that they follow the same classification. 
(6) The inclination of the asymptotes of a heterogeneous central curve is always the same as the inclination of the asymptotes of the curve represented by its highest homogeneous part. Because, if we transform to polar coordinates, and divide the polar equation by $r^{n}$, the resulting equation is of the form-

$$
\mathrm{F}_{0}(\cos \theta, \sin \theta)^{n}+\mathrm{F}_{1}(\cos \theta, \sin \theta)^{n-2} \cdot \frac{1}{r^{2}}+\mathrm{F}_{2}(\cos \theta, \sin \theta)^{n-4} \cdot \frac{1}{r^{4}} \ldots=\frac{1}{r^{n}} .
$$

Now, $r$ can only become infinite when $\mathrm{F}_{0}(\cos \theta, \sin \theta)^{n}=0$.

But this is also the condition for $r$ becoming infinite when the equation is reduced to its highest homogeneous part.

It follows that for all parallel sections of a central surface the inclination of the asymptotes to the axis of symmetry of the section is the same, and it is evident that all such asymptotes lie in two intersecting planes.

(7) In the case of the homogeneous central equations with an arbitrary term, it is evident that the curve cannot pass through the centre.

\section{Transformation to Secondary Axes-Rule of Signs.}

A symmetrical equation is evidently equiaxial; that is, the intercepts on the axes of reference are equal.

If a diametral symmetrical equation be transformed to secondary axes (bisecting the angles between the primary), the secondary axes are also diametral and symmetrical, and the curve consists of eight equal and similar segments. This might be inferred from general considerations as to symmetry, but it is desirable to prove it analytically. It may be here convenient to transcribe certain known formulæ of transformation of axes (with unchanged origin) of which $\mathrm{I}$ am to make use. If $\theta$ be the inclination of $\mathrm{X}$ to $x-$

$$
\begin{array}{ll}
\mathrm{X}=x \cos \theta-y \sin \theta & \mathrm{Y}=x \sin \theta+y \cos \theta \\
\mathrm{X}=(x-y) \cos \theta & \mathrm{Y}=(x+y) \sin \theta \\
\mathrm{X}=(x-y) \sqrt{\frac{1}{2}} & \mathrm{Y}=(x+y) \sqrt{\frac{1}{2}} .
\end{array} .
$$

(1) Is the formula for transformation in the same plane from any system of rectangular coordinates to any other rectangular system.

(2) Is the formula for transformation to symmetrical axes ; i.e., axes equally inclined to the original rectangular axes.

(3) Is the formula for transformation to axes which are at once symmetrical and rectangular, and which accordingly bisect the angles between the original rectangular axes; whence, $\cos \theta=\sin \theta=\sqrt{\frac{1}{2}}$.

In order to prove that a symmetrical diametral equation is of the same form when transformed to secondary axes, it is only necessary to write the generalised form of the expression in lines and columns. As the original axes are always supposed rectangular, 
the transformation to secondary axes is effected by substituting in every term the values $\sqrt{\frac{1}{2}}(x-y)$ for $X$ and $\sqrt{\frac{1}{2}}(x+y)$ for $Y$, and expanding. Let $A_{p}\left\{X^{n-p} Y^{p}+X^{p} Y^{n-p}\right\}$ be any pair of homologous terms ; their equivalent in the transformed equation is

$$
\left(\frac{1}{2}\right)^{\frac{n}{2}} \mathrm{~A}_{p}\left\{(x-y)^{n-p}(x+y)^{p}+(x-y)^{p}(x+y)^{n-p}\right\} \quad \text {. . . . . . . }
$$

Expanding the first term within the bracket in columns, the coefficients are-

$$
\begin{aligned}
& 1+\quad p+\frac{p \cdot(p-1}{1.2}+\frac{p \cdot(p-1)(p-2)}{1.2 .3}+\& \mathrm{c} . \\
&-(n-p)-(n-p) \times p-(n-p) \times \frac{p \cdot(p-1)}{1.2}-\& \mathrm{c} . \\
&+\frac{(n-p)(n-p-1)}{1.2}+\frac{(n-p)(n-p-1)}{1.2} \times p+\& \mathrm{c} .
\end{aligned}
$$

The coefficients of the expansion of the second term within the brackets are-

$$
\begin{array}{r}
1-p+\frac{p(p-1)}{1.2}-\frac{p \cdot(p-1)(p-2)}{1.2 .3}+ \\
+(n-p)-(n-p) \times p+(n-p) \times \frac{p \cdot(p-1)}{1.2}- \\
+\frac{(n-p)(n-p-1)}{1.2}-\frac{(n-p)(n-p-1)}{1.2} \times p+
\end{array}
$$

where in the first set of terms the sign $x$ is used to separate the factors derived from the expansion of $(x-y)^{n-p}$ from those derived from the expansion $(x+y)^{p}$, and similarly in the second set of terms.

The quantity in the first column (always unity) is the coefficient of $x^{n}$ in the transformed equation. The sum of the quantities in the second column is the coefficient of $x^{n-1} y$ in the transformed equation, and so on. We see that the expansion of the second term of the pair is the same as that of the first term, except that in the second, fourth, and every other alternate column, the signs + and - are interchanged, and therefore the sums of these columns in the two expansions is zero. If the pair of homologous terms have contrary signs, then in the expansion the sum of the first, third, \&c., columns is zero. From this analysis is derived the following abstract of results, hereafter referred to as the RULE of Signs.

1. From the mode of formation of the transformed equation it is always symmetrical if the original equation is symmetrical.

2. If in the original equation the terms constituting a symmetrical pair are of even degree and have like signs, i.e., both positive or both negative, then in the expansion of these terms in the transformed equation the sum of the partial coefficients is zero for all terms of uneven powers.

3. If in the original equation the terms constituting a symmetrical pair are of even 
degree with unlike signs, then in the expansion of these terms in the transformed equation the sum of the partial coefficients is zero for all terms of even powers.

4. If the sum of the indices in each term be uneven, then the expansion consists of a homogeneous expression containing only the even powers of one of the variables, and the uneven powers of the other variable. In my notation, if the terms are both positive, the transformed expression will consist of even powers of $y$ and uneven powers of $x$.

5. If we consider these equations only to be symmetrical where the terms of all the homologous pairs have like signs, or where for all homologous pairs the terms have unlike signs, then in the complete expansion of the transformed symmetrical equation of even degree, the sum of the partial coefficients is zero for uneven powers in the first case, and is zero for even powers in the second case ; in other words, if in the original equation, being of even degree, the homologous terms have like signs, the equation of the curve, when referred to secondary axes, consists entirely of terms of even powers. If in the original equation the homologous terms have contrary signs, the equation of the same curve, when referred to secondary axes, consists entirely of terms of uneven powers. These results are independent of the degree of the curve, and it will hereafter be shown that they are applicable to the projection of any symmetrical equation obtained by writing $x / a$ for $x$ and $y / b$ for $y$ (page 1069). Thus from the order of the signs of any symmetrical equation it is immediately known to which of the previously named classes the equation belongs, i.e., whether the curve represented is elliptic, hyperbolic, or inflexional.

6. These results are evidently true for any diametral, symmetrical equation, although not homogeneous, because it is only necessary to the proof that the equation should consist entirely of even or entirely of uneven terms.

\section{Diameters in Central Curves of the Fourth Degree.}

I shall now give a proof that every central curve of the fourth degree has two pairs of axes of symmetry, and in general only two such pairs.

(1) Let the equation be homogeneous, or of the form

$$
\mathrm{A} x^{4}+\mathrm{B} x^{2} y^{2}+\mathrm{C} y^{4}=1 \text {. . . . . . . }
$$

To prove that in general the curve has not a pair of conjugate axes equally inclined to a given line. Let the equation be referred to the given line and an axis perpendicular to that line. It may then be written

$$
\mathrm{D} x^{4}+\mathrm{E} x^{3} y+\mathrm{F} x^{2} y^{2}+\mathrm{G} x y^{3}+\mathrm{H} y^{4}=1 \quad \text {. . . . . }
$$

If we now transform to axes equally inclined to the line by formula (2) p. 1064, it will be seen whether the unknown angle $\theta$ can be determined so as to make the uneven terms of the resulting equation disappear, so that the resulting equation should be one referred to conjugate axes. To this effect we are to make the $x$ of equation $(2)=(x-y) \cos \theta$, and 
the $y$ of equation $(2)=(x+y) \cdot \sin \theta$, and expand in lines and columns. The expansion of the first term of (2) forms the first line, that of the second term is the second line, and so on.

$\begin{array}{llcccc} & x^{4} & x^{3} y & \frac{x^{2} y^{2}}{x y^{3}} & \frac{y^{4}}{} \\ \text { Term 1 } & \{1 & -4 & +6 & -4 & +1\} \mathrm{D} \cos \theta \\ \text { Term 1 } & \{1 & -2 & & +2 & -1\} \mathrm{E} \cdot \cos ^{3} \theta \sin \theta \\ \text { Term 3 } & \{1 & & -2 & & +1\} \mathrm{F} \cdot \cos ^{2} \theta \sin ^{2} \theta \\ \text { Term 4 } & \{1 & +2 & & -2 & -1\} \mathrm{G} \cdot \cos \theta \sin ^{3} \theta \\ \text { Term 5 } & \{1 & +4 & +6 & +4 & +1\} \mathrm{H} \cdot \sin \theta .\end{array}$

The coefficients of the new equation are of course the numerical quantities multiplied by the quantities outside the brackets. The two uneven terms in the new equations are accordingly

and

$$
\left\{-4 \mathrm{D} \cos \theta-2 \mathrm{E} \cos ^{3} \theta \sin \theta+2 \mathrm{G} \cos \theta \sin ^{3} \theta+4 \mathrm{H} \sin \theta\right\} x^{3} y,
$$

$$
\left\{-4 \mathrm{D} \cos \theta+2 \mathrm{E} \cos ^{3} \theta \sin \theta-2 \mathrm{G} \cos \theta \sin ^{3} \theta+4 \mathrm{H} \sin \theta\right\} x y^{3} .
$$

The coefficient of $x^{3} y$ cannot be changed into that of $x y^{3}$ by interchanging the signs + and - Hence a value of $\theta$ which makes the term $x^{3} y$ disappear will not in general make the term $x y^{3}$ disappear. In order that both terms may disappear, we must have $\mathrm{D}=\mathrm{H} ; \mathrm{E}=\mathrm{G} ; \cos \theta=\sin \theta$. Hence the condition of the existence of a pair of conjugate axes equally inclined to the axes of (2) is that the equation (2) be symmetrical. The conjugate diameters thus found are evidently the principal axes of symmetry of the curve.

If in equation (2) the second and fourth terms are supposed to be wanting, so that the curve is already referred to its principal axes of symmetry, then in the new equation the two uneven terms will be

$$
\{-4 \mathrm{D} \cos \theta+4 \mathrm{H} \sin \theta\} x^{3} y \text { and }\{-4 \mathrm{D} \cos \theta+4 \mathrm{H} \sin \theta\} x y^{3} \text {. }
$$

Their coefficients are identical, and the value, $\tan \theta=\mathrm{D} / \mathrm{H}$, will reduce both terms to zero, leaving an equation consisting of even powers, and therefore referred to conjugate diameters. The diameters thus found are the secondary axes of the curve; and the relation, $\tan \theta=\mathrm{D} / \mathrm{H}$ shows that they are the diagonals of the circumscribing parallelogram whose sides are parallel to the principal axes. When the highest power of $y$ is negative, a real solution is impossible, and the diagonals in question are the asymptotes of the hyperbolic curve of the fourth degree. These results might have been found directly by considering that every quartic homogeneous equation of even powers is necessarily a projection of an equiaxial form. Because the given equation, when referred to conjugate axes, is of the form

$$
\frac{x^{4}}{a^{4}}+p x^{2} y^{2}+\frac{y^{4}}{b^{4}}=1
$$

We have then only to take $\mathrm{P}=p a^{2} b^{2}$, or $\mathrm{P} / \alpha^{2} b^{2}=p$, in order to obtain the equation in projection form. 
(2) Where the central equation (being of the 4th degree) also contains terms of the 2nd degree $\mathrm{P}\left(x^{2}+y^{2}+x y\right)$ the term in $x y$ disappears by transformation, and the transformed expression consists entirely of even powers. The new axes are accordingly conjugate axes.

More generally an equation of any even degree, consisting of the highest terms of $x$ and $y$, and of one other term of even powers, may, in like manner, be immediately reduced to the projection of an equiaxial curve. Such a projection always has a pair of secondary axes, which are the diagonals of the circumscribing parallelogram.

\section{Diameters in Central Curves of Higher Degrees.}

For an equation of a degree higher than the fourth, secondary conjugate axes cannot in general be found. Because, if we transform and expand as before, there are for every even degree above the fourth more than two terms of uneven powers; and it is impossible, unless some relation amongst the coefficients be given, to determine $\theta$ so as to make the coefficients of more than two of the uneven terms vanish. The required relation is easily found. The equation must be a symmetrical function of $x / a$ and $y / b$. This being premised, if $\theta$ be the angle between $X$ and $x$, the inclination of the secondary axis, $x$, to the primary is given by the relation, $\tan \theta=b / a$. This will he made clear by two examples.

(13a). To find the Secondary Axes of the Curve $\mathrm{X}^{n} / a^{n} \pm \mathrm{Y}^{n} / b^{n}=1$.

(a) Taking first the upper sign, and transforming to axes equally inclined to the primary, by the formula $\mathrm{X}=(x-y) \cos \theta ; \mathrm{Y}=(x+y) \sin \theta$, we find for the transformed equation the expression

$$
\frac{\cos ^{n} \theta}{a^{n}}\left\{x^{n}-n x^{n-1} y+\frac{n(n-1)}{1.2} x^{n-2} y^{2}-\& c .\right\}+\frac{\sin \theta}{b^{n}}\left\{x^{n}+n x^{n-1} y+\& c .\right\}=1 .
$$

Let $\cos { }^{n} \theta / a^{n}=\sin ^{n} \theta / b^{n}$, or $\tan \theta=b / a$; then all the uneven terms disappear, and the equation is accordingly referred to conjugate diameters, which are equal in length, and symmetrically placed with reference the principal axes. These diameters coincide with the diagonals of the circumscribing parallelogram, whose sides are parallel to the principal axes of the oval of the $n^{\text {th }}$ degree.

(b) If the equation be taken with the negative sign, it is the terms of even powers which disappear in the transformation, and the secondary axes found are asymptotes of the hyperbolic curve.

(c) It is to be observed that the values above found for the inclination of the asymptotes of homogeneous symmetrical functions equated to an arbitrary term, are solutions of the relative functions equated to zero. Because the equation of the asymptote 
of a homogeneous curve is always that of the curve deprived of its arbitrary term. Hence $y / x=b / a$ is a solution of an equation in either of the forms

$$
\begin{gathered}
\frac{x^{n}}{a^{n}} \mp \frac{x^{n-2}}{a^{n-2}} \frac{y^{2}}{b^{2}} \mp \frac{x^{n-4}}{a^{n-4}} \frac{y^{4}}{b^{4}}-\frac{y^{n}}{b^{n}}=0 \\
\frac{x^{n}}{a^{n}} \mp \frac{x^{n-2}}{a^{n-2}} \mp \frac{x^{n-4}}{a^{n-4}} \mp+\frac{y^{n-4}}{b^{n-4}} \mp \frac{y^{n-2}}{b^{n-2}}-\frac{y^{n}}{b^{n}}=0 .
\end{gathered}
$$

If we divide the first of these by $y^{n}$ we obtain the form

$$
\frac{1}{a^{n}}\left(\frac{x}{y}\right)^{n} \mp \frac{1}{a^{n-2}} \frac{1}{b^{2}}\left(\frac{x}{y}\right)^{n-2} \mp \frac{1}{a^{n-4}} \frac{1}{b^{4}}\left(\frac{x}{y}\right)^{n-4} \mp \mp=1 .
$$

Treating $x / y$ as a single quantity $u$, we see that an equation of descending powers of $u$ equated to unity is always soluble, if its coefficients constitute a homogeneous function of $\alpha$ and $b$, in which case $a / b$ is a real root of the equation.

(13b). To find the Secondary Axes of any Curve of Symmetrical Expression referred to principal Axes.

The equation if homogeneous is of the form,

$$
x^{n} \pm \mathrm{A}_{2} x^{n-2} y^{2} \pm \mathrm{A}_{4} x^{n-4} y^{4} \pm \ldots \mp \mathrm{A}_{4} x^{4} y^{n-4} \mp \mathrm{A}_{2} x^{2} y_{-}^{n-2}+y^{n}=1
$$

In this symmetrical expression (after transformation to secondary axes equally inclined to the primary) every pair of homologous terms produces an expansion of the form (5) or (6) of p. 1065 above. The coefficients of the corresponding terms in the two expansions are equal, and the sum of the alternate columns is zero.

In the same way for curves of the form

$$
\mathrm{A}_{0} \frac{x^{n}}{a^{n}} \pm \mathrm{A}_{2} \frac{x^{n-2}}{a^{n-2}} \frac{y^{2}}{b^{2}} \pm \ldots \mp \mathrm{A}_{2} \frac{x^{2}}{a^{2}} \frac{y^{n-2}}{b^{n-2}} \pm \frac{y^{n}}{b^{n}}=1
$$

it may be shown, by taking $\bar{u}=x / a$, and $v=y / b$, that, when the curve is transformed by the formula for axes equally inclined to the primary axes, the coefficients of the alternate columns disappear, or are neutralised, when $\cos ^{n} \theta / \alpha^{n}=\sin ^{n} \theta / b^{n}$; or $\tan \theta=b / a$.

The solutions here given are applicable to symmetrical heterogeneous curves in any of the above forms, as may be verified by expanding separately the several homogeneous parts $u_{1} u_{2}$, \&c., which are of the above form; because in the proof of the Rule of Signs it is not assumed that the equation is homogeneous, but only that it consists of pairs of homologous terms equated to a constant. 


\section{Sextic Curves of the Homogeneous Form $\mathrm{F}(x, y)^{6}=\mathrm{A}^{6}$.}

The equation is understood to be referred to principal axes when it consists of terms of even powers only; but in the case of the symmetrical oval it will be seen that this description applies to each of the two pairs of conjugate axes; and there is, geometrically speaking, no reason why either pair should be considered principal axes preferentially to the other pair. The curves of this class do not pass through the centre.

In order to obtain fundamental forms, symmetrical equations are first to be considered. Of these there are in strictness only four species, corresponding to the four symmetrical combinations of the positive and negative signs of the terms. There are, for the sixth degree, two other forms, $(\gamma)$ and $(\delta)$, in which the coefficients are symmetrical, but the signs are not symmetrical. In the form $(\gamma)$ the extreme terms are positive, and the intermediate terms have unlike signs, the order of the signs being ++-+ . In the form $(\delta)$ the extreme terms have unlike signs and the intermediate terms have like signs, the order being +++- . The forms obtained by changing all the signs of the variables are, of course, the same curves. Also $(\gamma)$ is a variety of $(\beta)$, and $(\delta)$ is a variety of $(\zeta)$. As the equations are symmetrical, the equal coefficients of the highest powers disappear by division; the equations of the equiaxial curves of the sixth degree may then be written-

$$
\begin{aligned}
& x^{6}+\mathrm{P} x^{4} y^{2}+\mathrm{P} x^{2} y^{4}+y^{6}=\mathrm{A}^{6} \quad \cdot \quad \text {. . . . . . }(\alpha) \\
& x^{6}-\mathrm{P} x^{4} y^{2}-\mathrm{P} x^{2} y^{4}+y^{6}=\mathrm{A}^{6} \quad \text {. . . . . . . . . }(\beta) \\
& x^{6}+\mathrm{P} x^{4} y^{2}-\mathrm{P} x^{2} y^{4}+y^{6}=\mathrm{A}^{6} \quad \text {. . . . . . . . . }(\gamma)
\end{aligned}
$$

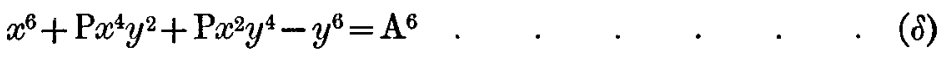

$$
\begin{aligned}
& x^{6}-\mathrm{P} x^{4} y^{2}+\mathrm{P} x^{2} y^{4}-y^{6}=\mathrm{A}^{6} \text {. . . . . . . . (є) } \\
& x^{6}+\mathbf{P} x^{4} y^{2}-\mathbf{P} x^{2} y^{4}-y^{6}=\mathbf{A}^{6} \quad \text {. . . . . . . . . }(\xi)
\end{aligned}
$$

Referring to p, 1062, where the principle of classification is indicated, $(a)$ is the Sextic: Oval; $(\beta)$ is the Inflexional Oval, passing into the Continuous Hyperbolic; $(\epsilon)$ is the Discontinuous Hyperbolic, consisting of alternate real and imaginary branches ; $(\gamma),(\delta)$, and $(\zeta)$ are Inflexional Hyperbolics.

(14a). To find the Equations of the Equiaxial Curves referred to Secondary Axes.

The coordinates of the original equations being denoted by capitals, if, in the formula of transformation, we were to make $\mathrm{Y}=(x+y) \sqrt{\frac{1}{2}}$, we should obtain negative values of $A^{6}$ in the transformed functions $(\epsilon)$ and $(\zeta)$. Therefore, let $X=(x+y) \sqrt{\frac{1}{2}}$; $\mathrm{Y}=(x-y) \sqrt{\frac{1}{2}}$, and transform to bisecting axes.

In the transformation of equations $(a)$ and $(\beta)$, we have for the sum of the 1 st and 4th terms,

$$
1 / 4\left\{x^{6}+15 x^{4} y^{2}+15 x^{2} y^{4}+y^{6}\right\}=\mathrm{X}^{6}+\mathrm{Y}^{6} \text {; }
$$


and for the sum of the 2nd and 3rd terms,

$$
1 / 4 \mathrm{P}\left\{x^{6}-x^{4} y^{2}-x^{2} y^{4}+y^{6}\right\}=\mathrm{PX}^{4} \mathrm{Y}^{2}+\mathrm{PX}^{2} \mathrm{Y}^{4}
$$

whence, by addition and subtraction we find for $\left(\alpha^{\prime}\right)$ and $\left(\beta^{\prime}\right)$ (being the curves, $\alpha$ and $\beta$, referred to their secondary axes).

$$
\begin{aligned}
& (1+\mathrm{P}) x^{6}+(15-\mathrm{P}) x^{4} y^{2}+(15-\mathrm{P}) x^{2} y^{4}+(1+\mathrm{P}) y^{6}=4 \mathrm{~A}^{6} \quad . \quad . \quad . \quad\left(\alpha^{\prime}\right) \\
& (1-\mathrm{P}) x^{6}+(15+\mathrm{P}) x^{4} y^{2}+(15+\mathrm{P}) x^{2} y^{4}+(1-\mathrm{P}) y^{6}=4 \mathrm{~A}^{6} \quad \text {. . . } \quad\left(\beta^{\prime}\right) \text {. }
\end{aligned}
$$

Again, in the transformation of $(\epsilon)$ and $(\zeta)$ we have for the sum of the two extreme terms,

$$
1 / 4\left\{6 x^{5} y+20 x^{3} y^{3}+6 x y^{5}\right\}=\mathrm{X}^{6}-\mathrm{Y}^{6},
$$

and for the sum of the two intermediate terms,

$$
1 / 4 \mathrm{P}\left\{\mp 2 x^{5} y \pm 4 x^{3} y^{3} \mp 2 x y^{5}\right\} \mp \mathrm{PX}^{4} \mathrm{Y}^{2} \pm \mathrm{PX}^{2} \mathrm{Y}^{4},
$$

where the upper and the lower signs belong respectively to the equations $(\epsilon)$ and $(\zeta)$.

Observing that the numerical coefficients are divisible by 2 , we have for $\left(\epsilon^{\prime}\right)$ and $(\zeta)$, by addition,

$$
\begin{aligned}
& (3-\mathrm{P}) x^{5} y+(10+2 \mathrm{P}) x^{3} y^{3}+(3-\mathrm{P}) x y^{5}=2 \mathrm{~A}^{6} \\
& (3+\mathrm{P}) x^{5} y+(10-2 \mathrm{P}) x^{3} y^{3}+(3+\mathrm{P}) x y^{5}=2 \mathrm{~A}^{6}
\end{aligned}
$$

The above are all the forms of symmetrical diametral equations that can be formed with four or three terms. If we seek for those that may be formed with only two terms, it is evident that

(1) The form $x^{6}+y^{6}=\mathrm{A}^{6}$, is a limiting form of $(\alpha)$ and $(\beta)$ when $\mathrm{P}=0 \quad \cdot \quad\left(a_{1}\right)$;

(2) $x^{6}-y^{6}=\mathrm{A}^{6}$, is a limiting form of $(\epsilon)$ and $(\zeta)$ when $\mathrm{P}=0 . \quad . \quad . \quad\left(\epsilon_{1}\right)$;

(3) $4 x^{4} y^{2}+4 x^{2} y^{4}=\mathrm{A}^{6}$, is a limiting form of $\left(\beta^{\prime}\right)$, and therefore of $(\beta)$ when $\mathrm{P}=1$, and is also a limiting form of $\left(\alpha^{\prime}\right),(\alpha)$, when $\mathrm{P}=-1 \quad . \quad . \quad\left(\beta_{2}\right)$;

(4) $4 x^{5} y+4 x y^{5}=A^{6}$, is a limiting form of $(\zeta)$, $(\zeta)$, when $P=5$, and of $\left(\epsilon^{\prime}\right),(\epsilon)$ when $\mathrm{P}=-5 \quad . \quad . \quad . \quad . \quad . \quad . \quad . \quad . \quad . \quad . \quad . \quad\left(\zeta_{2}\right)$.

Now we cannot directly obtain the last two forms with the negative sign from any of preceding equations. Hence, there are apparently two independent limiting forms,

$$
4 x^{4} y^{2}-4 x^{2} y^{4}=\mathrm{A}^{6} ;\left(\epsilon^{\prime \prime}\right) \text { and } 4 x^{5} y-4 x y^{5}=\mathrm{A}^{6}
$$

On further consideration, it is seen that $\left(\epsilon^{\prime \prime}\right)$ is derivable from $(\epsilon)$ or $\left(\epsilon^{\prime}\right)$, if the coefficients of the intermediate terms in the fundamental equations are supposed invariable, while the extreme terms, $x^{6}, y^{6}$ are supposed to be multiplied by coefficients which are indefinitely diminished. Transforming to secondary axes, we find

(1) The equation of $\left(\epsilon^{\prime \prime}\right)$ is unaltered in form and value by transformation $\left(\epsilon^{\prime \prime}\right)$

(2) The equation of $\beta^{\prime \prime}$ is $2 x^{5} y-4 x^{2} y^{3}+2 x^{\prime} y^{5}=1 . . \quad . \quad . \quad . \quad\left(\beta^{\prime \prime}\right)$, 
which is different from any of the previously given forms. But it has been found, as the result of the computation of values of $r$ and $\theta$, that this curve is identical with No. 10 of the table given below, which is of the form $(\beta)$; the explanation being that the curve consists of four real and four conjugate branches, and has accordingly four pairs of conjugate diameters.

As the result of a study of the fundamental forms here given, I have found that there are certain other critical values of $P$ which produce characteristic forms of the equiaxial curves. These I proceed to enumerate. From the drawings and relative tabular places (computed by the method of homogeneous variation) a very complete conception may be obtained of the possible variations of this family of sextic curves and their projections.

\section{(14b). Limiting Forms of the Equiaxial Curves.}

If $n$, the index number of a curve, be divisible into factors, $p$ and $q$, a symmetrical function of the $p^{\text {th }}$ or $q^{\text {th }}$ degree may be a limiting form of the symmetrical curve of the $n^{\text {th }}$ degree; for we have only to raise the equation of the $p^{\text {th }}$ degree to the power $q$, or to raise the equation of the $q^{\text {th }}$ degree to the power $p$ to obtain such a limiting form.

'Thus, (1) by raising the equation $x^{2} \pm y^{2}=\mathrm{A}^{2}$ to the 3rd power, we obtain the circle and the equilateral hyperbola in the sextic form,

$$
x^{6} \pm 3 x^{4} y^{2}+3 x^{2} y^{4} \pm y^{6}=A^{6}
$$

where, the upper sign being taken, we see that the circle is a limiting form of the equiaxial curve $(a)$, when $\mathrm{P}=3$. The lower sign being taken, the curve is the equilateral hyperbola, which is thus shown to be a limiting form of the equiaxial curve $(\epsilon)$ when $\mathrm{P}=3$. Similarly by writing $x / a$ for $x$, and $y / b$ for $y$, it may be shown that any ellipse or hyperbola is a limiting form of the sextic curve which is the projection of $(a)$, or $(\epsilon)$ to the principal axes $\alpha$ and $b$.

(2) It might be expected that a symmetrical cubic would also be a limiting form of an equiaxial sextic. This, however, is not universally true. I shall, however, write down the limiting forms obtained by squaring the symmetrical cubics $x^{3} \pm y^{3}=A^{3}$, and $x^{2} y \pm x y^{2}=\mathrm{A}^{3}$.

These forms, with the equivalent forms obtained by transforming to secondary axes, are as follows :-

$$
\begin{aligned}
& \left.\begin{array}{l}
\left(x^{3} \pm y^{3}\right)^{2} \\
x^{6} \pm 2 x^{3} y^{3}+y^{6}
\end{array}\right\}=\mathrm{A}^{6} ;\left\{\begin{array}{l}
x^{6}+6 x^{4} y^{2}+9 x^{2} y^{4} \\
y^{6}+6 y^{4} x^{2}+9 y^{2} x^{4}
\end{array}\right\}=2 \mathrm{~A}^{6} \\
& \left.\begin{array}{l}
\left(x^{2} y \pm y^{2} x\right)^{2} \\
x^{4} y^{2} \pm 2 x^{3} y^{3}+x^{2} y^{4}
\end{array}\right\}=\mathrm{A}^{6} \quad\left\{\begin{array}{l}
x^{6}-2 x^{4} y^{2}+x^{2} y^{4} \\
y^{6}=2 y^{4} x^{2}+y^{2} x^{4}
\end{array}\right\}=2 \mathrm{~A}^{6} .
\end{aligned}
$$

The forms in the second column are limiting cubic forms for a sextic curve referred to a transverse diameter $X$, and an asymptote $Y$, or the converse, as is made evident by dividing the equations by $x^{2}$ or $y^{2}$ respectively. 
(3) Another series of critical forms is the series where the coefficient $P=1$. By making $\mathrm{P}=1$ in the four equations $(\alpha),(\beta),(\epsilon)$, and $(\zeta)$ we obtain curves which may also be obtained by the multiplication of the factors $\left(x^{2}+y^{2}\right),\left(x^{2}-y^{2}\right)$ with $\left(x^{4}+y^{4}\right)$ and $\left(x^{4}-y^{4}\right)$. The forms $(\beta)$ and $(\zeta)$ are also obtained by the multiplication of $\left(x^{2} \pm y^{2}\right)^{2}$ with $\left(x^{2} \mp y^{2}\right)$.

(4) A fourth series of critical forms are those which correspond with the polar equations of sines and cosines of multiple arcs. These will be noticed in their order.

\section{(14c). Form and Variations of the Equiaxial Curves (a) and $(\beta)$.}

It is desirable to give a name to the variation of the curve consequent on the variation of the single coefficient $P$.

In equations of the 2nd degree the term "eccentricity" has relation to the variation due to projection, which is the only kind of variation of which these curves admit.

But in curves of the higher degrees, where we consider only those characteristics that are unaltered by projection, this kind of eccentricity is not considered at all. Hence, without ambiguity, I may make use of the term Quadrantal Eccentricity to denote the variation within each quadrant in the magnitude or direction of the secondary axes due to the variation of $P$, while the principal parameters remain unaltered.

The quadrants referred to are of course those which are marked out by rectangular reference lines, coinciding with the equal principal diameters or parameters of the curve.

Length of a Secondary Diameter in terms of the principal Diameter and P.-The variation of $\mathrm{P}$ in the oval of fourfold symmetry, has no effect on the direction of the secondary diameters, but only alters the ratio of their length to that of the primary. It is convenient always to put the length of a principal diameter $=1$, which is then also the value of the arbitrary term. The ratio of the secondary diameter to the primary may be denoted by $\Gamma$, which is also numerically the length of the former. In the oval forms of $(\alpha)$ and $(\beta)$ we then find for the length of the secondary diameters, by putting $x=y ; \quad \Gamma^{2}=2 x^{2}$, the relation,

$$
2 \mathrm{P}+2=\frac{1}{x^{6}}=\frac{2^{3}}{\Gamma^{6}} ; \therefore \frac{1}{\Gamma^{6}}=\frac{\mathrm{P}+1}{4} ; \mathrm{P}=\frac{4}{\Gamma^{6}}-1
$$

In curves of the hyperbolic type where the variation of $\mathrm{P}$ affects the direction of the asymptotes, the quadrantal eccentricity might be measured by the tangent of their inclination to the principal axis; but this relation has not been fully investigated.

Examples of the Curves, $(\alpha)$ and $(\beta)$.-The following equiaxial curves of the forms $(\alpha)$ and $(\beta)$ have been computed and traced. The number in the first column is a reference number corresponding with that in the second table; $P$ and $\Gamma$ are as above; 
$\mathrm{P}^{1}$ is the coefficient of the intermediate terms when the equation is transformed to secondary axes, the arbitrary term then being $\Gamma^{6}$.

\begin{tabular}{|r|r|r|c|}
\hline No. & \multicolumn{1}{|c|}{$\mathrm{P}$} & $\Gamma$. & Equation of Curve. \\
\hline 1. & 4 & 1 & $4 x y^{2}+4 x^{2} y=1$ \\
2. & 31 & $\sqrt{\frac{1}{2}}$ & $x^{6}+31 x^{4} y^{2}+31 x^{2} y^{4}+y^{6}=1$ \\
3. & 15 & $\left(\frac{1}{4}\right)^{1 / 6}$ & $x^{6}+15 x^{4} y^{2}+15 x^{2} y^{4}+y^{6}=1$ \\
4. & 7 & $\left(\frac{1}{2}\right)^{1 / 6}$ & $x^{6}+7 x^{4} y^{2}+7 x^{2} y^{4}+y^{6}=1$ \\
5. & 3 & 1 & $x^{6}+3 x^{4} y^{2}+3 x^{2} y^{4}+y^{6}=1$ \\
6. & 1 & $(2)^{1 / 6}$ & $x^{6}+x^{4} y^{2}+x^{2} y^{4}+y^{6}=1$ \\
7. & 0 & $(4)^{1 / 6}$ & $x^{6}+y^{6}=1$ \\
8. & $-\frac{1}{2}$ & $\sqrt{2}$ & $x^{6}-\frac{1}{2} x^{4} y^{2}-\frac{1}{2} x^{2} y^{4}+y^{6}=1$ \\
9. & -1 & $\infty$ & $x^{6}-x^{4} y^{2}-x^{2} y^{4}+y^{6}=1$ \\
10. & -5 & -1 & $x^{6}-5 x^{4} y^{2}-5 x^{2} y^{4}+y^{6}=1$ \\
\hline
\end{tabular}

No. 5 is the limiting circle. Nos. $1,2,3,4$ are the transformed equations $9,8,7$, and 6 , with the curves turned round through an angle of $45^{\circ}$. On referring to Plate I., where the numerals attached to the curves are those of the first column of the table, it is seen that starting from the circle, as $\mathrm{P}$ falls from 3 to 0 , the curve approaches more nearly to the circumscribing square (Pl. I. figs. 6, 7). For all negative values of $P$ between 0 and -1 the curve is inflexional (fig. 8), the secondary axis becoming more and more elongated, until at the value $\mathrm{P}=-1$ it passes into the continuous equilateral hyperbolic (fig. 9).

For values of $\mathrm{P}$ from -1 to -5 the curve is the discontinuous hyperbolic, where the angle between asymptote and axis ranges from $45^{\circ}$ to $22^{\circ} 30^{\prime}$, or $90^{\circ}$ to $45^{\circ}$ between the asymptotes.

In the diagram the curves $1,2,3,4$ are seen to be $9,8,7,6$, diminished and turned round through $45^{\circ}$.

For the value $\mathrm{P}=-5$ (No. 10 of the Table) we have four equal discontinuous hyperbolics, having angle between asymptotes $=45^{\circ}$ (Pl. II. fig. 2). The intervening angular spaces may be made to contain four equal and similar conjugate curves by changing the signs of all the variable terms in the equation. The equation of No. 10 referred to secondary axes contains only uneven powers (see No. 15). This curve also has the polar equation, $r^{6} \cos 4 \theta=\mathrm{A}^{6}$.

For negative values of $\mathrm{P}$ exceeding 5 we find hyperbolics of greater eccentricity, which are the conjugate curves of the series found for values of $P$ between -1 and -5 . The equation of these may also be obtained in another form, from the last-mentioned series, by changing the signs of all the variable terms. It is easily seen that a similar series of curves are obtained from the form $(\gamma)$, because one negative term suffices to make the oval inflexional.

The annexed table contains the places of $x$ and $y$ computed by the homogeneous method for curves 6 to 10 of the preceding table, so far as necessary, viz., from $0^{\circ}$ to $45^{\circ}$. 
From $45^{\circ}$ to $90^{\circ}$, the places are obtained by changing $x$ for $y$, and the places in the other three quadrants by changing the signs of $x$ and $y$.

Table of Computed Places.

\begin{tabular}{|c|c|c|c|c|c|c|c|c|}
\hline$\theta$ & $10^{\circ}$ & $15^{\circ}$ & $20^{\circ}$ & $25^{\circ}$ & $30^{\circ}$ & $35^{\circ}$ & $40^{\circ}$ & $45^{\circ}$ \\
\hline $\begin{array}{c}\text { Curve } \\
6 .\end{array}$ & $\begin{array}{l}x\left\{\begin{array}{l}0.9947 \\
y\end{array} \quad 0 \cdot 1754\right.\end{array}$ & $\begin{array}{l}0 \cdot 9877 \\
0 \cdot 2646\end{array}$ & $\begin{array}{l}0.9766 \\
0.3555\end{array}$ & $\begin{array}{l}0 \cdot 9603 \\
0 \cdot 4478\end{array}$ & $\begin{array}{l}0.9365 \\
0.5406\end{array}$ & $\begin{array}{l}0.9026 \\
0 \cdot 6320\end{array}$ & $\begin{array}{l}0.8557 \\
0.7180\end{array}$ & $\begin{array}{l}0.7936 \\
0.7936\end{array}$ \\
\hline $\begin{array}{c}\text { Curve } \\
7 .\end{array}$ & $\begin{array}{l}x\left\{\begin{array}{l}0.9999 \\
0.176\end{array}\right.\end{array}$ & $\begin{array}{l}0 \cdot 9998 \\
0 \cdot 268\end{array}$ & $\begin{array}{l}0.9996 \\
0.364\end{array}$ & $\begin{array}{l}0.9984 \\
0 \cdot 466\end{array}$ & $\begin{array}{l}0.994 \\
0.574\end{array}$ & $\begin{array}{l}0.982 \\
0.687\end{array}$ & $\begin{array}{l}0.951 \\
0.798\end{array}$ & $\begin{array}{l}0.891 \\
0.891\end{array}$ \\
\hline $\begin{array}{c}\text { Curve } \\
8 .\end{array}$ & $\begin{array}{l}x \\
y\end{array}\left\{\begin{array}{l}1.002 \\
0.177\end{array}\right.$ & $\begin{array}{l}1.007 \\
0.270\end{array}$ & $\begin{array}{l}1 \cdot 013 \\
0 \cdot 369\end{array}$ & $\begin{array}{l}1.022 \\
0.477\end{array}$ & $\begin{array}{l}1.037 \\
0.599\end{array}$ & $\begin{array}{l}1 \cdot 048 \\
0.734\end{array}$ & $\begin{array}{l}1 \cdot 049 \\
0.880\end{array}$ & $\begin{array}{l}1.0^{*} \\
1.0\end{array}$ \\
\hline $\begin{array}{c}\text { Curve } \\
9 .\end{array}$ & $\begin{array}{l}x\left\{\begin{array}{l}1 \cdot 006 \\
0.178\end{array}\right.\end{array}$ & $\begin{array}{l}1 \cdot 014 \\
0.272\end{array}$ & $\begin{array}{l}1.028 \\
0.374\end{array}$ & $\begin{array}{l}1 \cdot 051 \\
0 \cdot 490\end{array}$ & $\begin{array}{l}1.091 \\
0.630\end{array}$ & $\begin{array}{l}1 \cdot 171 \\
0 \cdot 820\end{array}$ & $\begin{array}{l}1 \cdot 373 \\
1 \cdot 152\end{array}$ & $\infty_{\infty}^{+}$ \\
\hline
\end{tabular}

\begin{tabular}{|c|c|c|c|c|c|c|c|c|c|}
\hline & $\theta$ & $5^{\circ}$ & $7^{\circ} 30^{\prime}$ & $10^{\circ}$ & $12^{\circ} 30^{\prime}$ & $15^{\circ}$ & $17^{\circ} 30^{\prime}$ & $20^{\circ}$ & $22^{\circ} 30^{\prime}$ \\
\hline Curve & $x$ & 1.007 & $1 \cdot 016$ & $1 \cdot 030$ & $1 \cdot 051$ & $1 \cdot 084$ & $1 \cdot 140$ & $1 \cdot 258$ & $\infty$ \\
10. & $y$ & 0.088 & 0.134 & $0 \cdot 182$ & 0.233 & 0.291 & 0.360 & 0.458 & $\infty$ \\
& $\log r$ & 0.005 & 0.010 & 0.019 & 0.032 & 0.050 & 0.078 & 0.127 & $\infty$ \\
\hline
\end{tabular}

(14d). Examples of the other Equiaxial Curves.

The following equiaxial curves of the forms $\epsilon$ and $\zeta$ have been computed and traced: the second column is a reference number, corresponding with that in the subjoined table of computed places: I, is the inclination of asymptote to transverse axis. The equation of each curve being given in different forms, those containing even powers are referred to a transverse axis $\mathrm{X}$; those containing odd powers are referred

* For No. 8, the following additional values are necessary to trace the second inflexion-

\begin{tabular}{|c|c|c|c|c|}
\hline & $41^{\circ}$ & $42^{\circ}$ & $43^{\circ}$ & $44^{\circ}$ \\
\hline$x$ & 1.045 & $\begin{array}{c}1.039 \\
0.935\end{array}$ & $\begin{array}{l}1.030 \\
0.961\end{array}$ & $\begin{array}{l}1 \cdot 016 \\
0.981\end{array}$ \\
\hline
\end{tabular}

+ For No. 9 we find also, $\theta=44^{\circ} 59^{\prime} ; x=4 \cdot 165 ; y=4 \cdot 163$. And for other curves additional places have been computed where necessary for tracing the inflexions.

VOL. XXXV. PART IV. (NO. 23). 
to an asymptote $X$; the polar equations in $\epsilon$ and $\zeta$ are referred to the transverse axis.*

\begin{tabular}{|c|c|c|c|c|}
\hline Class. & No. & I. & \multicolumn{2}{|l|}{ Equations of the Curves. } \\
\hline$\varepsilon$ & 11. & $45^{\circ}$ & $\left\{\begin{array}{l}x^{6}-x^{4} y^{2}+x^{2} y^{4}-y^{6} \\
x^{5} y+6 x^{3} y^{3}+x y^{5}\end{array}\right.$ & $\begin{array}{l}=1 \\
=1\end{array}$ \\
\hline$\epsilon$ & 12. & $15^{\circ}$ & $\left\{\begin{array}{c}x^{6}-15 x^{4} y^{2}+15 x^{2} y^{4}-y^{6} \\
\mp 6 x^{5} y \pm 20 x^{3} y^{3} \mp 6 x y^{5} \\
r^{6} \cdot \cos (6 \theta)=1: r^{6} \cdot \sin (6 \theta)\end{array}\right.$ & $\begin{array}{l}=1 \\
=1 \\
=1\end{array}$ \\
\hline $\begin{array}{l}\epsilon \\
\text { or } \\
\xi\end{array}$ & 13. & $45^{\circ}$ & $\left\{\begin{array}{l}x^{6}-y^{6}=1 \\
3 x^{5} y+10 x^{3} y^{3}+3 x y^{5}\end{array}\right.$ & $\begin{array}{l}=1 \\
=2\end{array}$ \\
\hline$\xi$ & 14. & $45^{\circ}$ & $\left\{\begin{array}{l}x^{6}+x^{4} y^{2}-x^{2} y^{4}-y^{6} \\
2 x^{5} y+4 x^{3} y^{3}+2 x y^{5} \\
r^{6} \cdot \cos (2 \theta)=1: r^{6} \cdot \sin (2 \theta)\end{array}\right.$ & $\begin{array}{l}=1 \\
=1 \\
=1\end{array}$ \\
\hline$\beta^{\prime \prime}$ & 15. & $22^{\circ} 30^{\prime}$ & $\left\{\begin{array}{l}4 x^{5} y-4 x y^{5} \\
4 x^{5} y^{\prime}-4 x^{1} y^{5} \\
r^{6} \cdot \sin (4 \theta)=1: r^{6} \cos (4 \theta)\end{array}\right.$ & $\begin{array}{l}=1 \\
=1 \\
=1\end{array}$ \\
\hline$e^{\prime \prime}$ & 16. & $\cdots$ & $\left\{\begin{array}{l}4 x^{4} y^{2}-4 x^{2} y^{4} \\
2 x^{5} y-4 x^{3} y^{3}+2 x y^{5}\end{array}\right.$ & $\begin{array}{l}=1 \\
=1\end{array}$ \\
\hline
\end{tabular}

The mode of variation of these curves is very remarkable; and it is the more deserving of attention, because it results from the rule of signs (p. 1065) that for any even degree

* The identity of the polar equations in the annexed tables with the Cartesian equations is proved as follows :--. $\operatorname{Cos}(2 \theta)=\cos ^{2} \theta-\sin ^{2} \theta$;

$\operatorname{Cos}(4 \theta)=\cos ^{4} \theta-6 \cos ^{2} \theta \cdot \sin ^{2} \theta+\sin ^{4} \theta$

$\operatorname{Cos}(6 \theta)=\cos ^{6} \theta-15 \cos ^{4} \theta \cdot \sin ^{2} \theta+15 \cos ^{2} \theta \cdot \sin ^{4} \theta-\sin ^{6} \theta$.

$\therefore$ (1) $\mathrm{A}^{6}=r^{6} \cdot \cos (2 \theta)=r^{4}\left(r^{2} \cdot \cos \overline{2} \bar{\theta}\right)=\left(x^{2}+y^{2}\right)^{2} \cdot r^{2}\left(\cos ^{2} \theta-\sin ^{2} \theta\right)$ $=x^{6}+x^{4} y^{2}-x^{2} y^{4}-y^{6}$. [No. 14 of Table.]

(2) $\mathrm{A}^{6}=r^{6} \cdot \cos (4 \theta)=r^{2} \cdot\left(r^{4} \cdot \cos \overline{4 \theta}\right)=\left(x^{2}+y^{2}\right) \cdot r^{4} \cdot\left(\cos ^{4} \theta-6 \cos ^{2} \theta \cdot \sin ^{2} \theta+\sin ^{4} \theta\right)$ $=x^{6}-5 x^{4} y^{2}-5 x^{2} y^{4}+y^{6}$. [No 10 of Table. $]$

(3) $\mathrm{A}^{6}=r^{6} \cdot \cos (6 \theta)=r^{6}\left(\cos ^{6} \theta-15 \cos ^{4} \theta \cdot \sin ^{2} \theta+15 \cos ^{2} \theta \cdot \sin 4 \theta-\sin ^{6} \theta\right)$ $=x^{6}-15 x^{4} y^{2}+15 x^{2} y^{4}-y^{6}$. [No. 12 of Table.]

Again, observing that-

$\operatorname{Sin}(2 \theta)=2 \sin \theta \cdot \cos \theta$

$\operatorname{Sin}(4 \theta)=4 \cos ^{3} \theta \cdot \sin \theta-4 \cos \theta \cdot \sin ^{3} \theta$; and

$\operatorname{Sin}(6 \theta)=6 \cos ^{5} \theta \cdot \sin \theta-20 \cos ^{3} \theta \sin ^{3} \theta+6 \cos \theta \cdot \sin ^{5} \theta$.

$\therefore$ (1) $\mathrm{A}^{6}=r^{6} \cdot \sin (\overline{2 \theta})=r^{4}\left(r^{2} \cdot \sin \overline{2 \theta}\right)=\left(x^{2}+y^{2}\right) 2 x y$ $=2 x^{5} y+4 x^{3} y^{3}+2 x y^{5}$. [No. 14 of Table.]

(2) $\mathrm{A}^{6}=r^{6} \cdot \sin (4 \theta)=r^{2}\left(r^{4} \cdot \sin 4 \theta\right)=\left(x^{2}+y^{2}\right)\left(4 x^{3} y-4 x y^{3}\right)$ $=4 x^{5} y-4 x y^{5}$. [No. 15 of Table.]

(3) $A^{6}=r^{6} \cdot \sin (6 \theta)=6 x^{5} y-20 x^{3} y^{3}+6 x y^{5}$. [No. 12 of Table. $]$ 
there is a series of equivalent curves which go through corresponding phases. The different forms are shown in Pl. II. figs. 1 to 4.

In their complete forms the equiaxial curves $(\epsilon)$ of the 6 th degree have three pairs of asymptotic axes; one pair being the secondary axes, whose inclination to the primary axes is always $45^{\circ}$; the other two pairs having an inclination depending on the value of $\mathrm{P}$ (fig. 3).

Table of Computed Places.

\begin{tabular}{|c|c|c|c|c|c|c|c|c|c|c|c|}
\hline $\begin{array}{c}\text { Curve } \\
11 .\end{array}$ & $\begin{array}{l}\theta \\
x \\
y\end{array}$ & & $\begin{array}{l}10^{\circ} \\
005 \\
177\end{array}$ & $\begin{array}{c}15^{\circ} \\
1 \cdot 012 \\
0.271\end{array}$ & $\begin{array}{c}20^{\circ} \\
1.021 \\
0.372\end{array}$ & $\begin{array}{c}25^{\circ} \\
1.034 \\
0.482\end{array}$ & $\begin{array}{c}30^{\circ} \\
1.054 \\
0.608\end{array}$ & $\begin{array}{c}35^{\circ} \\
1.079 \\
0.756\end{array}$ & $\begin{array}{c}40^{\circ} \\
1 \cdot 146 \\
0.961\end{array}$ & $\begin{array}{l}44^{\circ} 59^{\prime} \\
2 \cdot 470 \\
2 \cdot 468\end{array}$ & $\begin{array}{c}45^{\circ} \\
\infty \\
\infty\end{array}$ \\
\hline $\begin{array}{c}\text { Curve } \\
12 .\end{array}$ & $\begin{array}{l}\theta \\
x \\
y\end{array}$ & & $\begin{array}{l}2^{\circ} \\
.002 \\
.035\end{array}$ & $\begin{array}{c}4^{\circ} \\
1.013 \\
0.071\end{array}$ & $\begin{array}{c}6^{\circ} \\
\mathbf{1} \cdot 030 \\
0 \cdot 108\end{array}$ & $\begin{array}{c}8^{\circ} \\
1.059 \\
0 \cdot 149\end{array}$ & $\begin{array}{c}10^{\circ} \\
1 \cdot 106 \\
0 \cdot 195\end{array}$ & $\begin{array}{c}12^{\circ} \\
1 \cdot 190 \\
0.253\end{array}$ & $\begin{array}{c}14^{\circ} \\
1.414 \\
0.353\end{array}$ & \multicolumn{2}{|c|}{$\begin{array}{l}15^{\circ} \\
\infty \\
\infty\end{array}$} \\
\hline $\begin{array}{c}\text { Curve } \\
13 .\end{array}$ & $\begin{array}{l}\theta \\
x \\
y\end{array}$ & & $\begin{array}{l}10^{\circ} \\
.000 \\
176\end{array}$ & $\begin{array}{c}15^{\circ} \\
1 \cdot 000 \\
0.268\end{array}$ & $\begin{array}{c}20^{\circ} \\
1 \cdot 001 \\
0.364\end{array}$ & $\begin{array}{c}25^{\circ} \\
1.002 \\
0.467\end{array}$ & $\begin{array}{c}30^{\circ} \\
1.007 \\
0.581\end{array}$ & $\begin{array}{c}35^{\circ} \\
1.021 \\
0.715\end{array}$ & $\begin{array}{c}40^{\circ} \\
1.074 \\
0.901\end{array}$ & $\begin{array}{c}44^{\circ} 59^{\prime} \\
2 \cdot 461 \\
2 \cdot 460\end{array}$ & $\begin{array}{c}45^{\circ} \\
\infty \\
\infty\end{array}$ \\
\hline $\begin{array}{c}\text { Curve } \\
14 .\end{array}$ & $\begin{array}{l}\theta \\
x \\
y\end{array}$ & & $\begin{array}{l}5^{\circ} \\
9986 \\
0874\end{array}$ & $\begin{array}{c}10^{\circ} \\
0.9951 \\
0 \cdot 1755\end{array}$ & $\begin{array}{c}15^{\circ} \\
0.9895 \\
0.2652\end{array}$ & $\begin{array}{c}20^{\circ} \\
0.9824 \\
0.3576\end{array}$ & $\begin{array}{c}25^{\circ} \\
0.9754 \\
0.4548\end{array}$ & $\begin{array}{c}30^{\circ} \\
0.9721 \\
0.5611\end{array}$ & $\begin{array}{c}35^{\circ} \\
0.9797 \\
0.6860\end{array}$ & $\begin{array}{c}40^{\circ} \\
1.024 \\
0.861\end{array}$ & $\begin{array}{c}45^{\circ} \\
\infty \\
\infty\end{array}$ \\
\hline $\begin{array}{l}\text { Curve } \\
15 .{ }^{*}\end{array}$ & $\begin{array}{c}\theta \\
x \\
y \\
\log r\end{array}$ & $\begin{array}{l}0^{\circ} \\
\infty \\
0^{\circ} \\
\infty\end{array}$ & $\begin{array}{c}2^{\circ} 30^{\prime} \\
1 \cdot 338 \\
0.058 \\
0.127\end{array}$ & $\begin{array}{c}5^{\circ} \\
1 \cdot 191 \\
0 \cdot 104 \\
0 \cdot 078\end{array}$ & $\begin{array}{l}7^{\circ} 30^{\prime} \\
1 \cdot 113 \\
0 \cdot 147 \\
0 \cdot 050\end{array}$ & $\begin{array}{c}10^{\circ} \\
1.060 \\
0.187 \\
0.032\end{array}$ & $\begin{array}{l}12^{\circ} 30^{\prime} \\
1.021 \\
0 \cdot 226 \\
0.019\end{array}$ & $\begin{array}{c}15^{\circ} \\
0.989 \\
0.265 \\
0.010\end{array}$ & $\begin{array}{l}17^{\circ} 30^{\prime} \\
0.964 \\
0.304 \\
0.005\end{array}$ & $\begin{array}{l}22^{\circ} 3 \\
0.92 \\
0.38 \\
0.00\end{array}$ & \\
\hline $\begin{array}{c}\text { Curve } \\
16 .\end{array}$ & $\begin{array}{l}\theta \\
x \\
y\end{array}$ & $\begin{array}{l}0^{\circ} \\
\infty \\
0\end{array}$ & $\begin{array}{c}5^{\circ} \\
1 \cdot 786 \\
0 \cdot 156\end{array}$ & $\begin{array}{c}10^{\circ} \\
1 \cdot 421 \\
0.251\end{array}$ & $\begin{array}{c}15^{\circ} \\
1 \cdot 247 \\
0.334\end{array}$ & $\begin{array}{c}20^{\circ} \\
1 \cdot 139 \\
0 \cdot 414\end{array}$ & $\begin{array}{c}25^{\circ} \\
1.066 \\
0.497\end{array}$ & $\begin{array}{c}30^{\circ} \\
1.020 \\
0.589\end{array}$ & $\begin{array}{c}35^{\circ} \\
1.000 \\
0.700\end{array}$ & $\begin{array}{c}40^{\circ} \\
1.030 \\
0.865\end{array}$ & $\begin{array}{c}45^{\circ} \\
\infty \\
\infty\end{array}$ \\
\hline
\end{tabular}

In the form $(\epsilon)$, when $P=3$, the curve has the limiting form of the equilateral hyperbola (of 2nd degree), the three pairs of asymptotes being there coincident.

When $P>3$, there are six equal real branches, and six conjugate branches. If $P$ exceeds 3 by a very small quantity, the first real branch (bisected by $X$ ) is nearly rectangular, and the first conjugate branch is extremely acute. The secondary axis divides this from a similar acute real branch; and then there is a nearly rectangular conjugate branch bisected by $\mathrm{Y}$. When $\mathrm{P}=7$, the inclination of the first pair of asymptotes is

* These values of $\log r$ are identical with those of No. 10 of the preceding table. In No. 15 the curve is referred to asymptotes. 
$\pm 22^{\circ} 30^{\prime}$, and the first real branch is contained within an angle of $45^{\circ}$. The first conjugate branch and the second real branch are contained within angles of $22^{\circ} 30^{\prime}$; and the second conjugate branch (bisected by $\mathrm{Y}$ ) is contained within the angle $45^{\circ}$, and so on.

When $\mathrm{P}=15$ there are six real and six alternate conjugate branches all equal, each contained within an angle of $30^{\circ}$ (fig. 3). When $\mathrm{P}$ exceeds 15 , we have a series of pairs of unequal curves (which have not been fully investigated), but are probably the conjugates of the preceding set.

Returning to the neutral form of the equilateral hyperbola, and varying $\mathrm{P}$ by diminishing it indefinitely:-If $\mathrm{P}>0$ and $<3, x$ and $y$ can only become infinite for $\theta= \pm 45^{\circ}$; and we have a series of equilateral forms consisting of two real and two conjugate branches. The variation of $\mathrm{P}$ between these limits only affects the quadrantal eecentricity. The form $x^{6}-y^{6}=1$ is the limit between the forms $(\epsilon)$ and $(\zeta)$. In Pl. II. fig. (1) the curve which is nearest the centre is No. 13 of the Table; the curve furthest from the centre is the limiting equilateral hyperbola; and the intermediate curve is No. 11.

In the series (乌), where the signs of the two intermediate terms of the equation do not follow in alternate order, the curves are inflexional and equilateral, the only asymptotes being the secondary axes.

In Plate II. fig. 4, the curve which is nearest the centre is No. 14 of the Table. The curve next it, having the same pair of asymptotic axes, is traced from an equation of the same form with a different coefficient, $(P=15)$. The curve which has the axis of $\mathrm{X}$ for one of its asymptotes is evidently a limiting form of the same series, and is No. 16 of the Table. Its minimal radius-vector corresponds to-

$$
\theta=\tan ^{-1} \cdot 5=26^{\circ} 34^{\prime} \text {, nearly. }
$$

The variety $(\delta)$ resembles $(\zeta)$ in its forms and inflexions, but is not equilateral, as

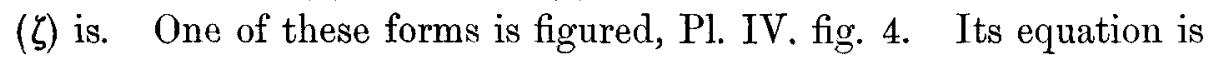

$$
x^{6}+x^{4} y^{2}+x^{2} y^{4}-y^{6}=1 ;
$$

and for the curve figured $(\mathrm{P}=1)$ the inclination of asymptotes to axis $\mathrm{X}$ (which depends on the value of $\mathrm{P}$ ) is $53^{\circ} 37^{\prime}$, nearly.

All the curves here traced have been computed by the tangent formula, which is the best for studying the transitions from one of the enumerated forms to the other or others.

General Results.-It is evident that the results which have been obtained are in the main independent of the degree of the symmetrical homogeneous equation. For equations of curves of even degree, referred to axes of symmetry, these results may be generalised as follows :-

(1) If all the terms are positive, the curve is an oval of fourfold symmetry, entirely concave to the centre, and having the circle as a limiting form. 
(2) If all the pairs of homologous terms of the symmetrical expression have like signs, but some of the pairs are positive and some are negative, the curve is the Inflexional oval, for all positive values of the coefficients which are less than those of the binomial expansion, and for all fractional negative values. Outside these limits the curve is a byperbolic, with alternate real and conjugate branches, the limit between the closed and open forms being the continuous hyperbolic in which all the branches are equal and real.

(3) If all the pairs of homologous terms have unlike signs, and if the equation when arranged in binomial form has the terms (being all even) alternately positive and negative, the curve consists generally of $n$ hyperbolic real branches, with alternating conjugate branches; but for certain values of the coefficients, the number may be reduced to two equilateral real branches, having the equilateral hyperbola as a limiting form.

(4) If all the pairs of homologous terms have unlike signs, but if the positive and negative terms do not follow in alternate order, the curve consists of two equilateral inflected branches, the curve being concave to the centre and to the asymptotes where it crosses the axis of $\mathrm{X}$, but after inflexion on either side of that axis becoming convex to the asymptotes.

The same form, where some of the pairs of terms have like signs, and some have unlike signs, except that the assymptotic axes are not rectangular.

(5) In all cases where the equation is reducible to the two-term Polar form,

$$
r^{n} \cos (p \theta)=1 \text {, }
$$

the curve consists of a number of alternate real and conjugate branches, which are all equal. The number of such forms evidently is $n / 2$, because $p$ may have the series of values, $n, n-2, n-4$, \&c.

(6) If the equation is not a symmetrical expression, but is homogeneous, the curves fall into the above categories, but have not in general secondary axes.

\section{Determination of Contour-lines of Homogeneous Surfaces.}

If $v_{1} v_{2}$ be coordinate quantities of any symmetrical diametral equation (suppose of the form $a$ ), and if $x^{2}+z^{2}$ be substituted for $v_{1}^{2}$, and $y^{2}+z^{2}$ for $v_{2}^{2}$, and the equation be expanded in terms of powers of $x^{2}, y^{2}, z^{2}$, we obtain the equation of a symmetrical homogeneous surface referred to conjugate diameters. The equation then takes successively the three forms which follow-

$$
\begin{aligned}
v_{1}^{6}+\mathrm{P} v_{1}^{4} v_{2}^{2}+\mathrm{P} v_{1}^{2} v_{2}^{4}+v_{2}^{6}=1 \\
\left(x^{2}+z^{2}\right)^{3}+\mathrm{P}\left(x^{2}+z^{2}\right)^{2}\left(y^{2}+z^{2}\right)+\mathrm{P}\left(x^{2}+z^{2}\right)\left(y^{2}+z^{2}\right)^{2}+\left(y^{2}+z^{2}\right)^{3}=1 \\
x^{6}+3 x^{4} y^{2}+3 x^{2} y^{4}+z^{6}+\mathrm{P}\left(x^{4} y^{2}+x^{4} z^{2}+2 x^{2} y^{2} z^{2}+2 x^{2} z^{4}+z^{4} y^{2}+z^{6}\right) \\
+\mathrm{P}\left(y^{4} x^{2}+y^{4} z^{2}+2 x^{2} y^{2} z^{2}+2 y^{2} z^{4}+x^{2} z^{4}+z^{6}\right)+y^{6}+3 y^{4} z^{2}+3 y^{2} z^{4}+z^{6}=1
\end{aligned}
$$

If we suppose the equation to be given in the form (3), we can only find values of 
$x, y, z$ for a central plane, and it is evidently impossible to determine a contour-line of the surface parallel to a central plane. Because if, for example, we make $z=\alpha$, the equation in $x$ and $y$ is thoroughly heterogeneous, containing in fact all the even terms of the general equation of the 6 th degree.

But if the equation be presented for solution in the form (2), we can find values of $x$ and $y$ in contour series. For we have then only to find a series of values of $v_{1}^{2} v_{2}^{2}$ (or $x^{2}+z^{2}$ and $y^{2}+z^{2}$ ); then, making $z=a$, we find from the series of values $v_{1,1} v_{2,1}$; $v_{1,2} v_{2,2} ; v_{1,3} v_{2,3}, \& c$. , the coordinates

$$
\begin{array}{llll}
x_{1}=\sqrt{v_{1,1}^{2}-a^{2}} ; & x_{2}=\sqrt{v_{1,2}^{2}-a^{2}} ; & x_{3}=\sqrt{v_{1,3}^{2}-a^{2}} ; & \& \mathrm{c} . \\
y_{1}=\sqrt{v_{2,1}^{2}-a^{2}} ; & y_{2}=\sqrt{v_{2,2}^{2}-a^{2}} ; & y_{3}=\sqrt{v_{2,3}^{2}-a^{2}} ; & \& \mathrm{c} .
\end{array}
$$

Through such a series of points a contour-line of the surface, in the plane $z=a$, may be traced.

In the same manner contour-lines may be traced for other planes parallel to $\mathrm{XY}$, viz. $z=b ; z=c, \& c$.

Such contour-lines have been computed and traced for surfaces derived from the curves $(\alpha)$ and $(\epsilon)$.

Plate VI. fig. 1, represents four contour-lines of the above surface (Eq. 2), with the coefficient $\mathrm{P}=1$. The values of $v_{1} v_{2}$ were taken from the preceding Tables (curve $a, 6$ ). The maximum value of $z$ was found to be, $z_{0}=7938$; and the three inner contour-lines were found by taking $z$ successively equal to $\frac{1}{2} z_{0}, \frac{2}{3} z_{0}$, and $\frac{3}{4} z_{0}$. The outermost contourline of this figure is the equatorial section of the surface, in the plane, $z=0$, and is identical with the curve of the Table, which is also figured in Pl. I.

It will be observed that as the circumference of the contour-lines decreases, the variation of curvature within the curve becomes less, the limiting form being evidently circular.

Plate VI. fig. 2, represents a series of contour-lines for the hyperbolic surface of two sheets, derived from $(\varepsilon, 12)$ of the Tables by writing $x^{2}+z^{2}$ for $v_{1}^{2}$ and $y^{2}+z^{2}$ for $v_{2}^{2}$. In this instance I have been less fortunate in the choice of contour-lines, because the lines are not far enough apart to give a clear notion of the figure of the surface. The values of $z^{2}$, from which the computations were made, are $\cdot 003, \cdot 0125$, and $\cdot 0275$, and the results are shown in the figure.

I may here observe that, while the preceding illustrations are confined to symmetrical forms, it is apparent that if the analytical expressions were varied by merely altering the coefficients of the terms, such a variation would only affect the symmetry of the curves, and would not in general produce a curve of a different type. There is no difficulty in forming any number of systems of unsymmetrical curves or contour-lines of surfaces, as we have only to fix on any unsymmetrical homogeneous expression in $v_{1} v_{2}$, and to replace these quantities by $\sqrt{a x^{2}+z^{2}}$ and $\sqrt{b y^{2}+z^{2}}$, giving such values to $z$ as may be desired; $x$ and $y$ are then found from $v_{1} v_{2}$. 


\section{Central Curves whose Equations are of the Form $\mathrm{F}_{1}(x, y)^{n}=\mathrm{F}_{2}(x, y)^{n-p}$.}

The first function may be divisible by the second, without remainder, the equation being then reducible to one of lower degree. This will generally be the case where the equation consists of pairs of homologous terms, all of which have like signs, or all unlike signs. I here suppose that the equation is not divisible.

Confining our attention, as before, to symmetrical diametral equations, it is evident that such equations always contain at least one uncombined power of the variables, because, if the equation be given in composite terms, we can always divide out the lowest powers of $x$ and $y$. When the equation after reduction consists of only two homogeneous parts its form is easily determined. Transforming to polar coordinates and dividing by the lowest power of $r$, we obtain an equation of the form $r^{n-p}=\frac{f^{p}(\cos \theta, \sin \theta)}{f^{n}(\cos \theta, \sin \theta)}$. The denominator of this fraction is formed from the terms of the highest homogeneous part, and if its terms be all positive, $r$ cannot become infinite; but if the numerator be wholly positive and the denominator contains positive and negative terms, there will be certain values of $\theta$ for which $r$ is infinite, these being the same as were found for the curves $\beta, \epsilon, \zeta$ (p. 1076). Again, if the numerator consists only of positive terms, the curve cannot pass through the origin, as it necessarily does where some of the terms in the numerator are positive and some are negative. If the terms of the highest homogeneous part be all positive, and the terms of the lower degree be partly positive and partly negative, the curve will be of the "foliated" type, consisting of a series of loops symmetrically arranged about the centre-origin, and having no inflexions except at the centre where the trace passes from one loop into another.

More generally, for symmetrical expressions of any even degree, and any number of pairs of homologous terms of even powers of the variables equated to zero ; which may be written $u_{n}+u_{n-2}+\ldots u_{2}=0$ : and are supposed to be reduced to their lowest terms,-

(1) If any pair or pairs of homologous terms of the part $u_{n}$ have unlike signs, while the terms of lower degree are all positive, or are all negative, then, by transforming to polar coordinates and dividing by $r^{n}$, we find that $r^{n}=\infty$ is a solution of the equation where $u_{n}=0$. The curve, therefore, consists of branches of infinite extent resembling those already described under the character of contour-lines of surfaces formed from the equations $(\epsilon)$ and $(\zeta)$.

(2) If $u_{n}$ consists entirely of positive terms, and if any pair or pairs of terms in the parts of lower degree have unlike signs, and the other pairs are all positive, then the curve consists of finite branches or loops passing through the centre. Because (1) the radius-vector cannot become infinite, since $u_{n}$ consists of positive terms, and (2) when $\cos \theta=\sin \theta$, all the negative terms are neutralised by the homologous positive terms, and there remains a series of positive terms equated to zero; whence $r=0$. In this case, since $\cos \theta=\sin \theta$ when $r=0$, the tangents at the centre bisect the angles between the axes of reference, and are secondary axes, and the centre is a point of inflexion. 
(3) A curve whose equation is strictly symmetrical, and consists of terms of even powers, whereof only one homologous pair have unlike signs, can have only two loops; but if any of the homogeneous expressions $u_{n-2}, u_{n-4}$, \&c., has a middle term, the curve may have a number of loops depending on the degree of the equation, because then the angle for which $r=0$ depends on a relation between three terms.

(4) A curve, consisting of loops passing through the centre, is also the result where $u_{n}$ is positive, and $u_{n-2}+u_{4}$, \&c., consists of pairs of positive terms and pairs of terms which are both negative; because evidently there must be definite values of $\theta$ which render $r=0$.

(5) If the terms $u_{n-2}+u_{n-4}, \&$ c., can be resolved into factors, while $u_{n}$ consists of pairs of unlike terms, the hyperbolic branches may break up into detached ovals sometimes with an infinite branch extending beyond these and within the same angular space.

These seem to exhaust the possible combinations for symmetrical equations without an arbitrary term.

(6) If we transform to axes equally inclined to the original symmetrical axes, the curve will be symmetrical about the new axes also, and the new equation will consist of even or of uneven powers of the variables, according to the rule of signs given above (p. 1065). In applying the rule, each homogeneous part of the equation is to be considered separately; so that, if one homogeneous part consist of positive terms, and the other of alternate positive and negative, their equivalents in the transformed equation will consist respectively of even and uneven powers.

(7) There are limiting parabolic forms where the highest homogeneous part contains only one of the variables, i.e., consists of a single term.

(8) In the case of axes which do not meet the curve except at the centre, these are, notwithstanding, true diameters, as the form of the equation proves. Accordingly, every such Exterior Diameter, if I may so term it, bisects the intercepts made by the adjacent branches or chords drawn parallel to the conjugate Exterior Diameter, and therefore bisects the Bitangents.

(9) These results are manifestly true, with the necessary restriction as to angles, for all projections of the curves in question.

(10) By an easy extension of (8) we have for all symmetrical equations of this type, and their projections, this relation: Each pair of Bitangents is parallel to one axis of symmetry, and is bisected by the axis conjugate to it.

\section{(16a). Examples of such Curves (Sixth Degree).}

Any of the functions on the left side of the sign of equality may be combined with any on the right; but of course the terms, when equated to zero, cannot all be positive.

The limits suited to this paper have been already so far exceeded that I shall not 
attempt to illustrate all the varieties. The following illustrations of curves of the 6 th degree of two homogeneous parts include the most characteristic forms :-

$$
\begin{aligned}
& \text { (1) } \left.x^{6}+3 x^{4} y^{2}+3 x^{2} y^{4}+y^{6}=x^{2}-y^{2}\right) \\
& \left.r^{4}=\cos 2 \theta\right\} \\
& \text { (1a) } \left.\begin{array}{rl}
x^{6}+3 x^{4} y^{2}+3 x^{2} y^{4}+y^{6} & =2 x y \\
r^{4} & =\sin 2 \theta
\end{array}\right\} \\
& \text { (2) } \left.\begin{array}{rl}
x^{6}+3 x^{4} y^{2}+3 x^{2} y^{4}+y^{6} & =x^{4}-6 x^{2} y^{2}+y^{4} \\
r^{2} & =\cos 4 \theta
\end{array}\right\} \\
& \text { (2a) } \left.\begin{array}{rl}
x^{6}+3 x^{4} y^{2}+3 x^{2} y^{4}+y^{6} & =4 x^{3} y-4 x y^{3} \\
r^{2} & =\sin 4 \theta
\end{array}\right\} \\
& \text { (3) } x^{6}-6 x^{4} y^{2}+6 x^{2} y^{4}-y^{6}=\mathrm{A}^{6}\left(x^{2}-y^{2}\right)
\end{aligned}
$$

The following Tables contain the computed places for the symmetrical half of a foliation or loop of each curve :-

Equation (1).

\begin{tabular}{rccccccccccc|}
\hline$\theta=$ & $0^{\circ}$ & $5^{\circ}$ & $10^{\circ}$ & $15^{\circ}$ & $20^{\circ}$ & $25^{\circ}$ & $30^{\circ}$ & $35^{\circ}$ & $40^{\circ}$ & $44^{\circ} \cdot 47$ & $45^{\circ}$ \\
$r=$ & 1.0 & 996 & 985 & 965 & 936 & 895 & 8441 & 765 & 646 & 363 & 0 \\
\hline
\end{tabular}

From $45^{\circ}$ to $135^{\circ}$ values of $r$ are impossible.

From $135^{\circ}$ to $180^{\circ}$ we obtain the above series reversed.

Similar results from $180^{\circ}$ to $360^{\circ}$.

The curve consists of two loops, and there are two inflexions at the centre.

Pl. III. fig. (1) is this curve, and fig. (2) is a projection of it.

\section{Equation (1a).}

\begin{tabular}{ccccccccccc|}
\hline$\theta=$ & $0^{\circ}$ & $5^{\circ}$ & $10^{\circ}$ & $15^{\circ}$ & $20^{\circ}$ & $25^{\circ}$ & $30^{\circ}$ & $35^{\circ}$ & $40^{\circ}$ & $45^{\circ}$ \\
$r=$ & 0 & 646 & 765 & 841 & .895 & 936 & 965 & .985 & 996 & 1.0 \\
\hline
\end{tabular}

$(1 a)$ is therefore (1) transformed to secondary axes, which are the tangents at the central point of inflexion.

Equation (2).

\begin{tabular}{ccccccccccc}
\hline$\theta=$ & $0^{\circ}$ & $3^{\circ}$ & $6^{\circ}$ & $9^{\circ}$ & $12^{\circ}$ & $15^{\circ}$ & $18^{\circ}$ & $21^{\circ}$ & $22^{\circ} \frac{3}{8}$ & $22^{\circ} \frac{1}{2}$ \\
$r=$ & 1. & 989 & 956 & .900 & .818 & 707 & 556 & 323 & 132 & 0 \\
\hline
\end{tabular}

Equation (2a).

\begin{tabular}{rlllllllll|}
$\theta=$ & $0^{\circ}$ & $1^{\circ} \cdot 5$ & $4^{\circ} \cdot 5$ & $7^{\circ} \cdot 5$ & $10^{\circ} \cdot 5$ & $13^{\circ} \cdot 5$ & $16^{\circ} \cdot 5$ & $19^{\circ} \cdot 5$ & $22^{\circ} \cdot 5$ \\
$r=$ & 0 & .323 & 556 & $\cdot 707$ & .818 & .900 & .956 & .989 & $1 \cdot 0$ \\
\hline
\end{tabular}


Equation (2a) represents the same curve as (2) transformed to axes inclined to the former at the angle $22^{\circ} \cdot 5$, and having the axes tangents at the centre origin. Values of $r$ are impossible for each alternate arc of $45^{\circ}$. The curve consists of four equal, similar, and symmetrical loops or foliations, and the centre is a point of inflexion for the four intersecting lines (fig. 3). Fig. (4) is a linear projection of the same curve.

In this and the preceding figure the bitangents are seen to be parallel to the conjugate axes.

The same construction is evident in fig. 4, which is a projection of the lastmentioned curve.

Equation (3).

\begin{tabular}{cccccccc|}
\hline$\theta=$ & $0^{\circ}$ & $10^{\circ}$ & $15^{\circ}$ & $20^{\circ}$ & $22^{\circ} \cdot 5$ & $23^{\circ}$ & $23^{\circ} \cdot 10$ \\
$r=$ & 1 & 1.03 & $1 \cdot 15$ & 1.37 & 1.68 & $3 \cdot 26$ & $\infty$ \\
\hline
\end{tabular}

Plate III. fig. 5, represents this curve, which consists of four hyperbolic branches without inflexions. As these branches do not pass through the centre, although $u_{2}$ contains a negative term, it is evident that the equation is reducible to one of lower degree with an arbitrary term. Accordingly, by division we find for the reduced form of equation $(3),-x^{4}+y^{4}-5 x^{2} y^{2}=\mathrm{A}^{6}$, or say, $=1$.

\section{Contour-lines of Surfaces derived from Central Curves passing through their Centres.}

Having already explained the mode of derivation of such lines, it is here only necessary to describe the illustrative figures (Pl. VI. figs. 3 and 4).

Figure 3 represents a surface with a central core or axis, being the axis of $Z$. It is formed from equation (1), above, by taking $r^{2}=r^{2}+z^{2} . \quad z_{0}=1$ is a maximum, and the four contour-lines are sections of the derived surface in the planes, $z=0, z=\cdot 65, z=\cdot 75$, and $z=\cdot 9$.

Figure 4 is formed from Equation 2 (above) by transforming to $x$-and- $y$ coordinates, and then taking $x^{2}=x^{2}+z^{\prime 2} ; y^{2}=y^{2}+z^{2} . \quad z_{0}=1$ is a maximum, and the contour-lines are for sections of the derived surface in the planes, $z=0, z=\cdot 1$, and $z=\cdot 14$.

In this surface the pear-shaped figures are only united at the cusps, which are also points of inflexion, and the sections consist of detached loops. The diagram makes clear what is the kind of variation of an equation by which a continuous looped curve may break up into detached loops or ovoids. We see that these only become continuous through the disappearance of the quantity $z$, and by the equation becoming a homogeneous function of $x$ and $y$ of the form,

$$
\mathbf{F}_{n}(x, y) / \mathbf{F}_{n-p}(x, y)=1
$$

It will be understood that these contour-lines are all traced from a sufficient number 
of computed values of $x$ and $y$, although I have not printed the tables of computed places.

\section{The Wave-Surface.}

This surface, as usually given, is of the form

$$
a^{2} x^{2} /\left(r^{2}-a^{2}\right)+b^{2} y^{2} /\left(r^{2}-b^{2}\right)+c^{2} z^{2} /\left(r^{2}-c^{2}\right)=0
$$

This, when cleared of fractions and expanded, is an equation of the 6th degree, containing all the terms of even powers of the general sextic equation of three variables.

If, however, the equation be merely cleared of its fractional form, and the terms be arranged in powers of $r$, it has the form

$$
\left\{a^{2} x^{2}+b^{2} y^{2}+c^{2} z^{2}\right\} r^{4}-a^{2} b^{2} c^{2} r^{4}+a^{2} b^{2} c^{2} r^{2}=0
$$

whence, dividing by $a^{2} b^{2} c^{2} r^{2}$, and writing $\alpha, \beta$, and $\gamma$, for $b^{2} c^{2}, c^{2} a^{2}$, and $\alpha^{2} b^{3}$, we have

where

$$
\left\{x^{2} / \alpha+y^{2} / \beta+z^{2} / \gamma\right\} r^{2}-r^{2}+1=0 ; . \quad . \quad . \quad . \quad .
$$

This may be written

$$
r^{2}=x^{2}+y^{2}+z^{2}
$$

$$
u_{4}-u_{2}+1=0
$$

where $u_{4}$ and $u_{2}$ are homogeneous functions of $x^{2}, y^{2}$, and $z^{2}$ (or of $x^{2}$ and $y^{2}$ in the plane curve), consisting entirely of positive terms.

The generalised form of the wave-surface, or wave-curve of any even degree, evidently is

$$
u_{m}+u_{n}+\ldots-u_{p}-u_{q}+1=0
$$

where $u$ is defined as above. The equation has an arbitrary term. The definition of $u$ implies that each homogeneous part of the equation consists of terms of like signs, and under this condition this equation of different homogeneous parts represents an oval (though it is usual only to consider the semi-oval) entirely concave to the centre. If any of the homogeneous parts $u_{p}$ should consist of a homologous pair of negative terms and a homologous pair of positive terms, the curve would be the inflexional oval (Pl. IV. fig. 5); but, as already seen, so long as each homologous pair of terms have like signs, $r$ can neither become 0 or $\infty$; and the curve or surface is always and necessarily a continuous closed curve of double symmetry.

Plate III. (fig. 7) is a representation of the limiting form of the 4 th degree, obtained from equation (1) by suppressing the 3rd term. The reduced equation is

$$
a^{2} x^{4}+\left(a^{2}+b^{2}\right) x^{2} y^{2}+b^{2} y^{4}=a^{2} b^{2} r^{2}
$$


whence

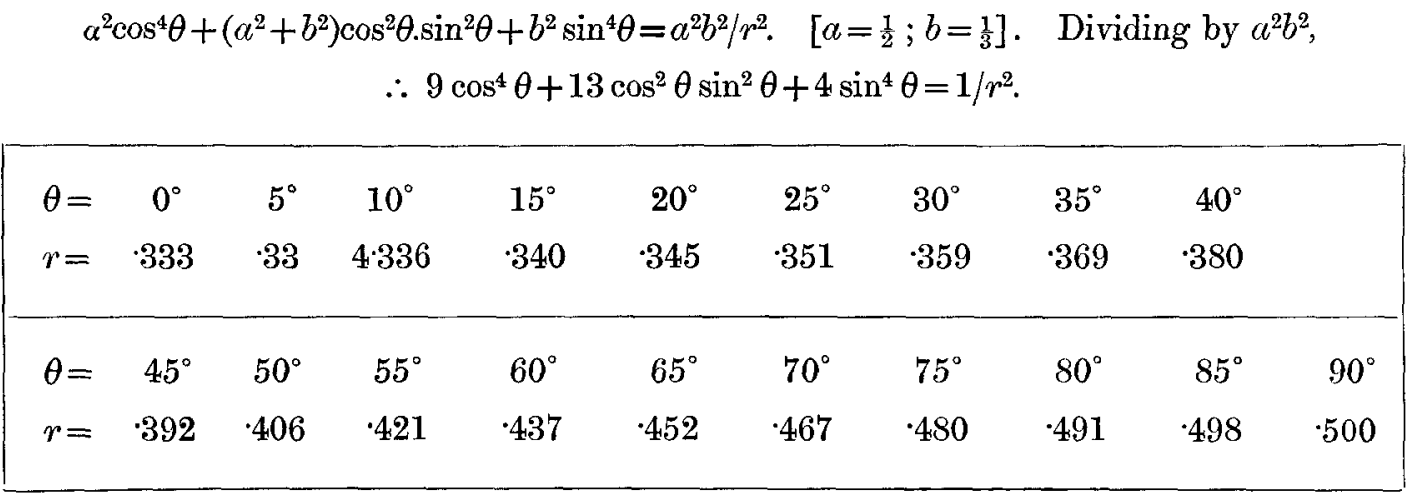

Returning to the equation of the wave-surface in its usual form (1), the curve of any section through an axis, $Z$, is most easily computed by transforming to polar coordinates. If, as usual, we make $x=r \cos \theta \cdot \cos \phi ; y=r \sin \theta \cdot \cos \phi ; z=r \sin \phi$; and then divide by $r^{2} \cos ^{2} \phi$, we obtain

$$
\left(\frac{c^{2}-r^{2}}{c^{2}}\right)\left\{\left(\frac{a^{2} \cdot \cos ^{2} \theta}{r^{2}-a^{2}}\right)+\left(\frac{b^{2} \sin ^{2} \theta}{r^{2}-b^{2}}\right)\right\}=\tan ^{2} \phi
$$

whence $\phi$ may be found for any given values of $r$ and $\theta$.

In the following illustration, Pl. III. fig. (6), I suppose a section through $Z$ making the angle

$$
\theta=45 ; \sin \theta=\cos \theta=\sqrt{\frac{1}{2}} \cdot c=3 ; b=2 ; a=1 .
$$

The form of the equation shows that $r$ must be $>2 ;<3$.*

\begin{tabular}{cccccccccccccc}
\hline$=$ & $2 \cdot 0$ & $2 \cdot 05$ & $2 \cdot 1$ & $2 \cdot 2$ & $2 \cdot 3$ & $2 \cdot 4$ & $2 \cdot 5$ & $2 \cdot 6$ & $2 \cdot 7$ & $2 \cdot 8$ & $2 \cdot 9$ & $3 \cdot 0$ \\
$\theta=$ & $90^{\circ}$ & $73^{\circ} \cdot 2$ & $58^{\circ}$ & $47^{\circ} \cdot 1$ & $39^{\circ} \cdot 7$ & $33^{\circ} \cdot 8$ & $28^{\circ} \cdot 7$ & $24^{\circ} \cdot 2$ & $19^{\circ} \cdot 9$ & $15^{\circ} \cdot 5$ & $10^{\circ} \cdot 5$ & $0^{\circ}$
\end{tabular}

The two curves are shown in figs. (7) and (6), and although the first is of the 4th degree and the 2 nd is of the 6th degree, the resemblance is very apparent. These may be compared with the curve of Pl. II. fig. (5), which represents the symmetrical equation

$$
x^{4}+x^{2} y^{2}+y^{4}=\frac{1}{4}\left(y^{2}+y^{2}\right)
$$

\section{Curves Symmetrical about One Axis.}

It has been observed that an ordinary section of a central surface is only central when it is taken parallel to a principal plane. But now, if a central section be taken in any direction through an axis of symmetry $(\mathrm{Y})$ of the central surface, then all sections parallel to this will be symmetrical about $y$, but will not necessarily or usually be symmetrical

* The numerical equation is $\frac{9-r^{2}}{18 r^{2}-18}+\frac{18-2 r^{2}}{9 r^{2}-36}=\tan ^{2} \varphi$. 
about the axis perpendicular to $Y$. To obtain a curve of single symmetry from any central surface referred to conjugate axes XYZ, we have only to transform to new axes $x$ and $z$, leaving $\mathrm{Y}$ unaltered. If we then make $z=$ unity, or any arbitrary value, within proper limits, we obtain an equation in $x$ and $y$, which is the required equation. If the given central surface have all its terms positive, then the form of the curves of the oblique sections (parallel to an axis) resembles that of the Cartesian oval; that is, it is a symmetrical closed curve without inflexions, but more pointed towards the positive direction of $x$ than towards the negative.

I shall give an illustration of such a curve of the 6th degree. Let the surface equation be

$$
\mathrm{X}^{6}+\mathrm{Y}^{6}+\mathrm{Z}^{6}=1 .
$$

This, when transformed to secondary axes in the plane $\mathrm{XZ}$ has the equation

$$
x^{6}+15 x^{4} z^{2}+15 x^{2} z^{4}+z^{6} y^{6}=1
$$

In the equation, as first given, let $\mathrm{Y}$ remain unchanged, and let the equation be tranformed to axes $x$ and $z$, each having the inclination $60^{\circ}$ to the original plane XY. The formula of transformation is

$$
\mathrm{X}=(x-z) \cdot \cos 60^{\circ} ; \mathrm{Z}=(x+z) \cdot \sin 60^{\circ} .
$$

If in the transformed equation $z$ be taken equal to unity, and the equation cleared of fractions, the resulting expression for the plane curve is

$$
16 y^{6}=9-\left\{7 x^{6}+39 x^{5}+105 x^{4}+130 x^{3}+105 x^{2}+39 x\right\},
$$

where the new arbitrary term, 9 , is the difference between $z^{6}$ or unity and the arbitrary term of the transformed surface equation. The new plane $x y$ is then inclined at $60^{\circ}$ to $\mathrm{XY}$.

The following values of $x$ and $y$ have been computed :-

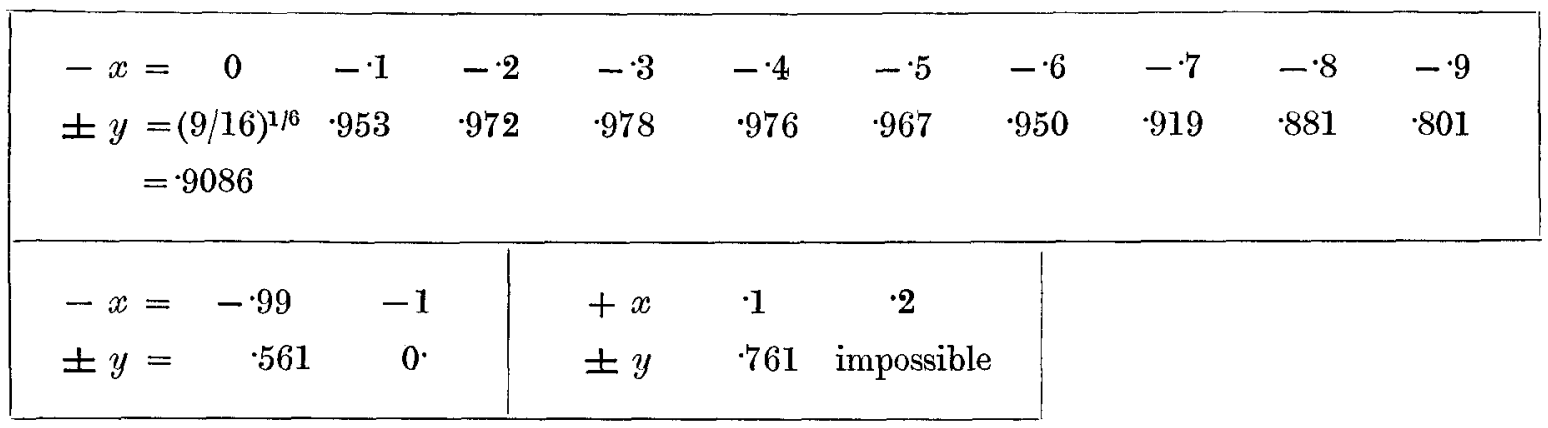

The approximate value of $+x$ when $y=0$ is $\cdot 154$.

'The value $-x=-1$, when $y=0$, is exact.

If the equation of the derivative surface contains the terms $\mathrm{P} x^{4} y^{2}+\mathrm{P} x^{2} y^{4}$, the equation of the section may contain additional terms of the form $y^{2}\left(x^{4}+x^{3}+x^{2}+x\right)$ and $y^{4}\left(x^{2}+x\right)$, where the coefficients are omitted. 
If in the original equation $y^{6}$ be taken negative, the surface will be a sextic hyperboloid of one sheet, $y$ being the axis. Taking a section whose inclination to the original plane $\mathrm{XY}$ is $60^{\circ}$, we obtain the same expression for the plane curve as that last given, except that the terms on the right side of the sign of equality have their signs changed.

The following values of $x$ and $y$ have been computed for this hyperbolic curve of single symmetry: the first value only being approximate, those from 2 to $2 \cdot 0$ being exact.

$\begin{array}{llcccccc}+x=\cdot 154 & \cdot 2 & \cdot 4 & 6 & .8 & 1 \cdot 0 & 2 \cdot 0 & \infty \\ \mp y=0 & .80 & 1 \cdot 14 & 1.35 & 1.54 & 1.72 & 2.60 & \infty\end{array}$

The values of $x$ of the immediately preceding table give an opposite and dissimilar branch.

The two curves are shown in figs. (1) and (2) of Pl. IV.

(19a). To find a Symmetrical Expression for the Oval of Single Symmetry.

Referring to the figures of the curves, since for every pair of equal positive and negative values of $y$ there are two values of $x$, it is always possible to inscribe a square in such a figure. Let the oval be referred to the diagonals of the inscribed square as coordinate axes. Then the equation must satisfy three conditions:-(1) The uncombined terms of $x$ and $y$ are all terms of even powers, otherwise the values of $+x$ and $-x$ would not be equal when $y=0 ;(2)$ the equation is a symmetrical expression, because the axes of reference are equally inclined to the axis of symmetry; (3) the terms are all positive, because, according to the rule of signs (above), it is only positive pairs of even terms which, when transformed to bisecting axes, produce exclusively even powers of $y$, as must be the case here. There is a fourth condition. I may here anticipate what is proved in the section on radial coordinates, that the algebraic equations of these curves, when referred to their axes of symmetry, contain no uneven powers in the even terms; i.e., these even terms are of the form $x^{2 p} y^{2 q}$, and we have already shown that the transformation to axes having the inclination $45^{\circ}$, does not introduce uneven powers. Hence (4) our symmetrical equation may consist of pairs of the terms $x^{6}, y^{6} ; x^{4} y^{2}, x^{2} y^{4} ; x^{2} y^{2}$ and $x^{2}, y^{2}$ together with composite uneven terms. If an equation satisfying these conditions be made homogeneous by supplying powers of $z$, it is seen that the axes of the plane curve lie in principal planes of the homogeneous closed symmetrical surface, and that the origin of the plane curve is in a diameter of this surface.

Unless all the uneven terms are present, the oval will be inflexional. See figure (3) of Plate IV.

Similar results are obtained for the hyperbolic curve of single symmetry.

These curves are best investigated by means of radial equations from two foci, as given in the sequel. The origin or point to which the last equation is referred has no 
direct relation that I can discover to the foci. It is certainly not the mean point between the foci in the curve of single symmetry, because then the radial equation would be a symmetrical expression with equal coefficients, which is of course not the case in curves of single symmetry.

It is evident that the sextic hyperboloid of one sheet, and indeed a similarly constructed surface of any degree, will furnish either oval or asymptotic curves of the quasiCartesian type, according to the angle at which the section is taken.

If the equation of such a surface contains only the highest power of one of the positive terms, $x$, then when $x^{n}=$ the arbitrary term, the equation reduces to a pair of right lines or rules. But the number of such rules apparently cannot exceed that of the conjugate diameters for a hyperboloid of any even degree above the second.

\section{(19b). Examples of Curves of Single Symmetry.}

To form the equation of the oval of single symmetry of any degree, it is not necessary to go through the process of forming a surface equation and then transforming to new axes. I have only done this to illustrate the theorem that every plane curve is a section of a homogeneous surface of the same degree.

From the mode of formation of the preceding expressions, it is easily seen that a symmetrical binomial function of $x$ and $z$, with the highest power of $y$ added, becomes an oval of single symmetry when a definite value is given to $z$, as in the following illustration :-

$$
\mathrm{P}(x+z)^{8}=\mathrm{P}\left\{x^{8}+8 x^{7} z+28 x^{6} z^{2}+56 x^{5} z^{3}+70 x^{4} z^{4}+56 x^{3} z^{5}+28 x^{2} z^{6}+8 x z^{7}+z^{8}\right\} \pm y^{8}=1,
$$

By making $z=1$, we obtain

$$
\mathrm{P}(x+z)^{8}=\mathrm{P}\left\{x^{8}+8 x^{7}+28 x^{6}+56 x^{5}+70 x^{4}+56 x^{3}+28 x^{2}+8 x\right\}+\mathrm{P}-1=\mp y^{8} .
$$

If all the signs of the second equation be changed, then the positive sign of $y$ gives the closed oval, and the negative sign of $y$ the asymptotic form.

In this equation for any possible value of $x$, the positive and negative roots of $y$ are equal; but for a given value of $y$ the roots of $x$ are unequal.

On these considerations the following methods have been devised for obtaining the curves of single symmetry of any degree (1). In any diametral homogeneous equation in $v_{1} v_{2}$ we may take $v_{1}=z+\sqrt{x} ; v_{2}=y ; \therefore x=\left(v_{1}-z\right)^{2}$; whence values of $x$ and $y$ are found from $v_{1} v_{2}$ for any required value of $z$. Or we may take $v_{2}^{2}=y^{2} ; v_{1}^{2}=z^{2}+x$, whence $x=v_{1}^{2}-z^{2}$; and the equation consists of even powers of $y$ and uneven powers of $x$.

The curve of Pl. VI. fig. 7 , which is of the form of a rifle-ball, was obtained from $(\alpha, 6)$ by substituting $5+\sqrt{x}$ for $v_{1}$ after transforming the origin to the extremity of the axis of $\mathrm{X}$. It is of the 12th degree. I might have taken $z+x^{3 / 2}=0$, or $x^{3}=v_{1}-z$.

Each of the homogeneous curves, $a, \beta, \epsilon$, and $\zeta$, may be made to furnish by deriva- 
tion curves of single symmetry of different degrees. Again, by giving different values to $z$ in any of the derived equations, a series of contour-lines may be traced representing a surface which is symmetrical about one axis.

(2) An equation also represents a curve of single symmetry when it is of the homogeneous form $u_{n} / u_{n-p}=1$, and (1) the function $u_{n}$ is of even, and $u_{n-p}$ of uneven degree, and also (2) the terms contain only even powers of one of the quantities, $y$. Thus the equation

$$
x^{6}+\mathrm{P} x^{4} y^{2}+\mathrm{P} x^{2} y^{4}=x^{5}-\mathrm{Q} x^{3} y^{2}+\mathrm{R} x y^{4} \text {, }
$$

represents a non-central symmetrical equation, from which contour-lines of a derived surface may be obtained by substituting $x^{\prime}+z$ for $x$ and $y_{2}^{\prime}+z^{2}$ for $y$ in the equation, and finding values of $x^{\prime}$ and $y^{\prime}$ from $x$ and $y$ for any required value of $z$.

I ought, perhaps, to refer here to the case of curves composed of factors; but the subject has been already fully investigated; and I could scarcely hope to add anything material to what has been found by writers of higher authority in these matters. I only make this observation, that when equations expressed in terms of factors are expanded in terms connected by additive or subtractive signs, it is generally necessary to give alternative signs to some of the terms, consistently with the original equation, otherwise the complete ovals will not be obtained. I have given an illustration of such a curve of the 6 th degree, in which the expansion of the terms of factors does not lead to alternative signs, and which forms an elegant symmetrical closed curve having thirty-two inflexions in its orbit. Its equation is

$$
\left(x^{2}-1\right)\left(x^{2}-2\right)\left(x^{2}-3\right)+\left(y^{2}-1\right)\left(y^{2}-2\right)\left(y^{2}-3\right)=1 . \quad(\text { Pl. IV. fig. 5. })
$$

\section{Parabotic Limiting Forms.}

Considered as a section of the homogeneous central surface, a parabola of the $n^{\text {th }}$ degree is evidently a section of such a surface parallel to any tangent plane of the asymptotic cone. For such a section the inclination of the asymptotes (which is the same as that of a parallel section through the centre) vanishes, which proves that the curve is parabolic. It is not quite correct to describe a parabola (as is sometimes done) as being a curve whose equation wants the highest power of one of the variables. Homogeneous equations are always central curves, although they may not contain the highest powers of both variables. An equation in $x, y$, represents a parabolic curve when one of the variables does not occur in the highest homogeneous part; in other words, when the highest part consists of a single term, $y^{n}$, or is reducible to a single term by transformation of axes. Because, by transforming to polar coordinates, and dividing by $\sin ^{n} \theta$, we see that when $\sin \theta$ is equated to zero $r$ becomes infinite, and that there are no asymptotes, because $u_{n}$ consists of a single, term. When in the equation of a parabola of any degree, $u_{n}$ consists of more than one term, then since $u_{n}$ must be derived by transformation of axes from a single term, $y^{n}$ (where $y^{\prime}$ becomes $p x+q y$ ) the homogeneous part, $u_{n}$ ought to be a 
complete square, or a complete binomial expansion of $p x+q y$. This would seem to be the proper criterion by which an equation not referred to principal axes may be known to be the expression of a parabolic curve.

If the equation consists entirely of terms of even powers, there are two parabolas, one on each side of the axis of $\mathrm{X}$, which may in a sense be considered to be two branches of a central curve, as in the following easy example:-

\begin{tabular}{|ccccccc|}
\multicolumn{10}{c|}{$y^{6}+4 y^{4}=4 x^{4}$} \\
\hline$y=0$ & 1 & 2 & 3 & 4 & 5 & 6 \\
$x=0$ & 1.057 & 2.379 & 4.027 & 5.981 & 8.206 & 10.670. \\
\hline
\end{tabular}

\section{Biradial Coordinates.}

The homogeneous equations hitherto treated have been solved for loci determined by Cartesian coordinates. The same equations and the same series of values as are above found may be represented graphically under different coordinate systems, and so as to produce curves differing widely in form and geometric properties from the curves of the $x$-and- $y$ system.

If to avoid ambiguity we call the coordinates for which values were found $v_{1}, v_{2}$, these may represent radii, angles, or trigonometrical quantities instead of lines drawn to coordinate axes; and the equations may be equations in $r_{1}$ and $r_{2} ; \theta_{1}$ and $\theta_{2} ; r$ and $x$; or $r$ and $z$ (where $r$ is the radius vector from a pole, and $z$ is a perpendicular on a directrix). This last system again may be immediately transformed into trilinear coordinates by substituting $x^{2}+y^{2}$ for $r^{2}$. The homogeneous equation in $\sin \theta_{1}$ and $\sin \theta_{2}$ is evidently identical with the homogeneous equation of corresponding terms in $r_{2}$ and $r_{1}$; because in the variable triangle composed of the two radii and the line joining the foci the sides are proportional to the sines of the opposite angles.

As an illustration of what may be done in a new direction with the homogeneous equations already examined, the following chapter on a class of Biradials has been written :-

The radial coordinates, from foci $F_{1}, F_{2}$, are denoted by $r_{1} r_{2}$; and the distance $\mathrm{F}_{1} \mathrm{~F}_{2}$ by $2 c$.

The equations here considered are of the form

$$
r_{1}^{n} \pm r_{2}^{n}=\mathrm{A}_{1}^{n} \quad(1) ; \quad r_{1}^{n} / \lambda^{n} \pm r_{2}^{n} / \mu^{n}=c^{n} / \nu^{n}=\mathrm{A}^{n} \quad .
$$

where the index $n$ is an even number.

I have not been able to come to a clear conclusion regarding biradial equations of uneven degrees. On the one hand, if we seek to transform these to rectangular or ordinary polar coordinates, it is necessary to square the equation twice to remove the VOL. XXXV. PART IV. (NO. 23). 
radical, so that a biradial equation of the 3rd degree corresponds to an equation of the 12th degree in rectangular Cartesian coordinates; while a biradial of the 4th degree can be transformed into a quadric in Cartesian coordinates. On the other hand, when the biradial curves of the $3 \mathrm{rd}, 4 \mathrm{th}, 5 \mathrm{th}$, and 6 th degrees are traced, they are found to be in series; and in this case, apparently, the degree of the Cartesian equation (contrary to the general understanding of mathematicians on this subject) is not a criterion of the true order of the curve.

Plate V. fig. 8, represents this series of curves, that of the 3rd degree being the nearest to the centre. The dotted curve is the biradial curve of the fractional degree 7/2. Each curve is laid down from nine computed points for the quadrant.

Equation (1) is the Oval of double symmetry. Its equation in ordinary polar coordinates reduces to a simple and easily remembered expression. Taking $O$, the centre of the oval for the origin of Cartesian and polar coordinates $(x, y, \mathrm{R}, \theta) ; \mathrm{OF}_{\mathbf{1}}=\mathrm{OF}_{2}=c$. For a point $\mathrm{P}$ in the curve, we have from the triangles $\mathrm{OF}_{1} \mathrm{P}, \mathrm{OF}_{2} \mathrm{P}$,

$$
\begin{aligned}
& r_{1}^{2}=\mathrm{OP}^{2}+\mathrm{OF}_{1}^{2}-2 \mathrm{OF}_{1} \cdot \mathrm{OP} \cdot \cos \mathrm{F}_{1} \mathrm{OP}=\mathrm{R}^{2}+c^{2}-2 c \mathrm{R} \cos \theta \\
& r_{2}^{2}=\mathrm{OP}^{2}+\mathrm{OF}_{2}^{2}+2 \mathrm{OF}_{2} \cdot \mathrm{OP} \cdot \cos \mathrm{F}_{2} \mathrm{OP}=\mathrm{R}^{2}+c^{2}+2 c \mathrm{R} \cos \theta
\end{aligned}
$$

In the expansions of $r_{1}^{n}, r_{2}^{n}$ the uneven terms of $\mathrm{R} \cos \theta$ disappear. Thus by transformation $r_{1}^{2}+r_{2}^{2}=\mathrm{A}^{2}$ becomes

$$
\begin{gathered}
\left(\mathrm{R}^{2}+c^{2}-2 c \mathrm{R} \cdot \cos \theta\right)+\left(\mathrm{R}^{2}+c^{2}+2 c \mathrm{R} \cdot \cos \theta\right)=\mathrm{A}^{2} \\
\therefore \quad \mathrm{R}^{2}=\left(\mathrm{A}^{2}-2 c^{2}\right) / 2, \text { the circle. }
\end{gathered}
$$

For the radial equation of the 4th degree we have

$$
\begin{gathered}
r_{1}^{4}+r_{2}^{4}=\mathrm{A} ; \quad\left(\mathrm{R}^{2}+c^{2}-2 c \mathrm{R} \cdot \cos \theta\right)^{2}+\left(\mathrm{R}^{2}+c^{2}+2 c \mathrm{R} \cdot \cos \theta\right)^{2}=\mathrm{A}^{4} \\
\left(\mathrm{R}^{2}+c^{2}\right)^{2}+4 c^{2} \mathrm{R}^{2} \cos ^{2} \theta=\mathrm{A}^{4} / 2 \\
\therefore \quad\left(x^{2}+y^{2}+c^{2}\right)+(2 c x)^{2}=\mathrm{A}^{4} / 2
\end{gathered}
$$

For the radial equation of the 6th degree we have

$$
\begin{aligned}
r_{1}^{6}+r_{2}^{6}=A^{6} ; & \left(\mathrm{R}^{2}+c^{2}-2 c \mathrm{R} \cos \theta\right)^{3}+\left(\mathrm{R}^{2}+c^{2}+2 c \mathrm{R} \cdot \cos \theta\right)^{3}=\mathrm{A}^{6}, \\
\therefore \quad & \left(\mathrm{R}^{2}+c^{2}\right)^{3}+\left(\mathrm{R}^{2}+c^{2}\right)(2 c \mathrm{R} \cos \theta)^{2}=\mathrm{A}^{6} / 2 ; \\
\therefore \quad & \left(x^{2}+y^{2}+c^{2}\right)^{3}+\left(x^{2}+y^{2}+c^{2}\right)(2 c x)^{2}=\mathrm{A}^{6} / 2
\end{aligned}
$$

Cognate polar equations may be formed in the same way for radial equations of any even degree, whence the equations in $x$ and $y$ may be written out. The equations are homogeneous functions of the composite quantities $\left(x^{2}+y^{2}+c^{2}\right)$ and $2 c x$.

If we write $v_{1} v_{2}$ for these expressions, and solve the homogeneous equation in $v_{1} v_{2}$ for any point, we may then find $x=v_{2} / 2 c$, and $y=\sqrt{v_{1}-x^{2}-c^{2}}$. 
These equations do not presuppose any relation between $\mathrm{A}$ and $c$. Accordingly, by varying the distance of $\mathrm{F}$ and $\mathrm{O}$, the equation may be made to represent an oval from any two points on the major or minor axis taken as poles, and these poles may be either interior to, or on the oval, or (within certain limits) exterior to it. If $a$ and $b$ represent the lengths of the principal semi-axes of the oval, the limiting position of an exterior pole or focus is $c=2 a$, where the curve is reduced to a line. The limiting positions of interior poles or foci is of course $c=0$, where the foci coincide, and the curve becomes a circle.

The principal foci of the oval are determined under the same conditions as the foci of an ellipse; by taking $c^{2}=a^{2}-b^{2}$. Then, for the pair of equal radii drawn from the foci to the extremity of the minor axis, we have the relation $r_{1}=r_{2}=\alpha$. If the major axis be taken as of unit value, then $c^{2}=\left(\alpha^{2}-b^{2}\right) / a^{2}=e^{2}$, and the polar equations for the 4 th and 6th degree curves may be written

$$
\begin{aligned}
& \mathrm{R}^{4}+2 e^{2} \mathrm{R}^{2}\left(1+2 \cos ^{2} \theta\right)=\left(\mathrm{A}^{4}-2 e^{4}\right) / 2 \\
& \mathrm{R}^{6}+\left(3 e^{2} \mathrm{R}^{4}+3 e^{4} \mathrm{R}^{2}\right)\left(1+4 \cos ^{2} \theta\right)=\left(A^{6}-2 e^{6}\right) / 2
\end{aligned}
$$

where $e$ is the eccentricity estimated in the same manner as in the case of the ellipse, the cognate curve of the 2nd degree. And similarly for any curve of even degree in which the foci are properly taken. It may here be noticed that the equation $\mathrm{R}^{2}\left(1+c^{2} \cos ^{2} \theta\right)=\mathrm{A}^{2}$ represents an ellipse, because it may be immediately changed to $y^{2}+c^{2} x^{2}=\mathrm{A}^{2}$. The ellipse then belongs to this family of curves, of which it is of course the lowest form.

Plate V. fig. 4, represents the sextic curve having the equation

$$
r_{1}^{6}+15 r_{1}^{4} r_{2}^{2}+15 r_{1}^{2} r_{2}^{4}+r_{2}^{6}=1
$$

and referred to its principal foci. For its construction the values of $r_{1} r_{2}$ are used, which are transcribed in the ensuing table, p. 1096. But as the curve was to be referred to its principal foci, it was necessary to adopt as the maximum and minimum radii the pair of values whose sum is equal to twice the mean radius, $r_{21}$. Hence the only available values were

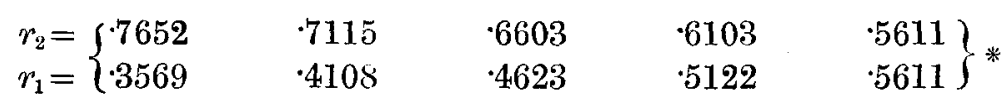

The curves here considered have a general resemblance to ellipses; and if the equation in $x$-and-y coordinates be referred to oblique axes, the curve resembles an ellipse referred to conjugate inclined axes. The greatest and least diameters are thus apparently conjugate, but are not really so; because it has been found impossible by analysis to reduce the locus of mid-points of parallel chords to a simple equation. It will be seen from Plate V. that the difference between the biradial curve of the 6 th degree and the ellipse described on the same axes is very small, and it is probable that the class of homo-

\footnotetext{
* These coordinates are very nearly the same as those of the ellipse described on the same axes.
} 
geneous biradial curves may be resolved into functions of curves of the second degree with variable elements.

Hitherto I have considered the radial curve as the geometrical expression of a homogeneous radial equation of the simple form $r_{1}^{n}+r_{2}^{n}=\mathrm{A}$. It will now be shown that all symmetrical homogeneous equations in $r_{1} r_{2}$ of the same degree are identical curves, the eccentricity being dependent only on the choice of foci, or, which is the same thing, on the ratio of $c$ to $A$. This identity is proved by transformation to polar coordinates. For this purpose, let $r_{1}^{p} \cdot r_{2}^{n-p}$ and $r_{1}^{n-p} . r_{2}^{p}$ be any pair of homologous terms of the symmetrical radial equation. If definite values be given to the indices $n$ and $p$, and the transformation to polar coordinates be effected by the formula $r_{1,2}=\mathrm{R}^{2} c^{2} \pm 2 \mathrm{R} c \cdot \cos \theta$, it will be found that, in the addition of the transformed terms, all the terms of uneven powers disappear, and that the resulting polar expression is identical with that obtained from the sum of the terms $r_{1}^{n}$ and $r_{2}^{n}$. Thus, from each pair of homologous terms we have the same polar expression multiplied by a coefficient, and the transformed homogeneous symmetrical radial equation has the form already found for the equation of three terms, with a new value of $A$.

Thus, if we transform the equation $r_{1}^{6}+\mathrm{P} r_{1}^{4} r_{2}^{2}+\mathrm{P} r_{1}^{2} r_{2}^{4}+r_{2}^{6}=\mathrm{A}^{6}$ to polar coordinates, we obtain from the two extreme terms the terms of the left side of equation (4), which may be denoted by $\mathrm{F}\left(r_{1} r_{2}\right)$; and from the mean terms we obtain $\mathrm{P} \times \mathrm{F}\left(r_{1} r_{2}\right)$. The polar equation then is of the form (4) with the right-hand term divided by $(P+1$.$) , or$

$$
\left(\mathrm{R}^{2}+c^{2}\right)^{3}+\left(\mathrm{R}^{2}+c^{2}\right)(2 c \mathrm{R} \cos \theta)^{2}=\frac{\mathrm{A}^{6}}{2 \mathrm{P}+2}
$$

The proposition that the same curve or trace may be obtained from different homogeneous equations of the same degree is illustrated by fig. (1). This figure, as is the case with all the illustrations, is drawn by tracing the curve through a series of computed points laid down on diagram paper, never less than nine points for a complete phase. This figure, when referred to the two marked exterior foci, satisfies the equation $r_{1}^{6}+r_{1}^{4} r_{2}^{2}+r_{1}^{2} r_{2}^{4}+r_{2}^{6}=1$; and when referred to the two marked interior foci it satisfies the equation $r_{1}^{6}+r_{2}^{6}=1$. The computed values of $r_{1}$ and $r_{2}$ are those of the table, p. 1074, for the equations of these forms in $x$ and $y$.

If the homogeneous radial equation contains a middle term of even powers of $r_{1} r_{2}$, its equivalent in polar coordinates differs only from the expression found for a pair of homologous terms in having the negative sign prefixed to all the terms containing $\cos ^{2} \theta$. I must, therefore, qualify the statement of the preceding paragraph by adding that, in the case of homogeneous equations of the 4th and 8 th degrees (and generally where the index is divisible by 4), there are apparently two forms, one without and the other with a middle term. But it does not appear that this variation can have any other effect than that of varying the coefficients of the terms multiplied by $\operatorname{cosin} e^{2} \theta$. The examples which I have worked out are confined to equations of the 6th degree, in which, of course, there is no middle term. 
If the equation consists of only a middle term equated to unity, or $r_{1}^{\frac{n}{2}} \cdot r_{2}^{\frac{n}{2}}=1$, this is immediately reducible to $r_{1}^{2} \cdot r_{2}^{2}=1$, which gives by transformation,

$$
\left(x^{2}+y^{2}+c^{2}\right)^{2}-4\left(x^{2}+y^{2}\right) c^{2} \cos ^{2} \theta
$$

the equation of the oval of Cassini.

There is a curious relation between radial equations and equations of the same form in rectangular coordinates, which is connected with the value of the coefficients.

It has been seen (p. 1072), in the case of the sextic equation of positive even powers of $x$ and $y,(1)$ that if $\mathrm{P}$, the coefficient of the pair of intermediate terms be $=3$, the curve is a limiting circle ; (2) that by varying $P$ from 0 to 3 we obtain every form of the noninflexional oval; and (3) that for values of $P$ exceeding 3 and less than 15 , or $n(n-1) / 2$, we obtain the same series of curves turned round through an angle of $45^{\circ}$. In the case of the sextic radial equation of positive even powers of $r_{1} r_{2}$, if $\mathrm{P}$, the coefficient of the pair of intermediate terms $=3$, the curve also reduces to the circle $\left(r_{1}^{2}+r_{2}^{2}=1\right)$. If $\mathrm{P}$ be less than 3, the radial equation represents a curve referred to foci, or poles, in the line of the minor axis, which may be either interior or exterior or on the curve (see figs. 1 and 2); and this includes the case of the equation of three terms, where $P=0$. But, if $P$ exceeds 3 , the radial equation represents a curve referred to foci, or poles, in the line of the major axis, which may be either interior or exterior or on the curve; and this includes the case of the curve referred to its principal foci.

It appeared to me that the radial curves, as traced, were a little more rounded at the apses than ellipses, and this impression has been confirmed by the numerical computation and comparison of the forms of the ellipse and of the sextic oval described on identical major and minor axes, which will be immediately given. It would be interesting to make a cognate comparison for elliptic ovals of different degrees. There are two ways in which such a comparison may be instituted.

(1) If the vertices of the curves be taken for foci, or poles of radial coordinates, the arbitrary term is then a parameter, and a series of curves of different degrees may be described upon the same principal axes, $a$ and $b$. (2) If the foci of the normal position $\left(c^{2}=\alpha^{2}-b^{2}\right)$ be taken for poles, it is difficult to prearrange the equations so that the curves to be formed shall have the same amplitude. We may, however, compute each curve independently, and compare it with the ellipse described on the same axes, and thus find out for the curve of any degree how far its coordinates differ from those of the ellipse of equal amplitude. In either case, it is necessary to reduce the radial coordinates to rectangular. This is easily done. Referring to p. 1092, we see that

Also,

$$
r_{2}^{2}-r_{1}^{2}=4 c \mathrm{R} \cos \theta=4 c x ; \therefore x=\frac{r_{2}^{2}-r_{1}^{2}}{4 c} \text {. }
$$

$$
r_{2}^{2}+r_{1}^{2}=2\left(x^{2}+y^{2}+c^{2}\right) ; \therefore y^{2}=\frac{r_{2}^{2}+r_{1}^{2}}{2}-\left(x^{2}+c^{2}\right)
$$


To determine $a$ and $b$, and $c$ (the distance between the foci), we may denote by $r_{0,2} \quad r_{0,1}$ the greatest and least radial coordinates, being those which are drawn to the extremities of the axis of $\mathrm{X}$; and by $r_{2, \mathrm{1}}$ the radii of equal length, being those which are drawn to the extremities of the axis of $\mathrm{Y}$. Then,

For any interior foci, . . . $\quad c=\frac{r_{02}-r_{01}}{2} ; a=\frac{r_{02}+r_{01}}{2}$;

For exterior foci, . . . . . . $\quad c=\frac{r_{02}+r_{01}}{2} ; a=\frac{r_{02}-r_{01}}{2}$;

For foci on the curve, . . . $r_{01}=0 ; c=a=\frac{r_{02}}{2}$;

For foci in the normal position $r_{02}+r_{01}=2 r_{21}=2 \alpha$; and $r_{21}-r_{01}=c$;

In all cases, . . . . . $b=\sqrt{r_{21}^{2}-c^{2}}$.

Values of $a, b, c, x$ and $y$ being thus found for the elliptic oval of any degree from its radial coordinates $r_{1} r_{2}$, the comparison with the ellipses described on the same axes is made by taking identical values of $x$ (or $x=x^{\prime}$ ), and thence computing the relative values of

$$
y^{\prime}=\sqrt{b^{2}-\frac{b^{2}}{a^{2}} \cdot x^{2}} .
$$

Such a comparison has been made for the curve $r^{6}+15 r_{1}^{4} r_{2}^{2}+15 r_{1}^{2} r_{2}^{4}+r_{2}=1$, and the ellipse having the same axes, $a$ and $b$; and the values of $y$ and $y^{\prime}$ to the argument $x=x^{\prime}$, together with the difference $\left(\Delta=y-y^{\prime}\right)$ are given in the subjoined table. Exact values of $r_{1} r_{2}$ were found by the homogeneous method, whence exact values of $x, y$, and $y^{\prime}$ were found by the preceding formulæ. Taking the highest and lowest computed values of $r_{2} r_{1}$ for the axial radii, and with interior foci, we have $r_{02}=\cdot 9367 ; r_{01}=\cdot 1651 ; c=\cdot 3858$; $a=5509 ; b=\cdot 4075$. From these elements and the tabular values of $r_{2}$ and $r_{1}$, the corresponding values of $x, y$, and $y^{\prime}$ are as in the annexed table.

\begin{tabular}{|c|c|c|c|c|c|c|c|c|}
\hline Aronuments $\left\{r_{2}\right.$ & $\cdot 9367$ & 8798 & $\cdot 8215$ & $\cdot 7652$ & $\cdot 7115$ & $\cdot 6603$ & 6103 & 5611 \\
\hline$\left\{r_{1}\right.$ & $\cdot 1651$ & $\cdot 2358$ & $\cdot 2990$ & 3569 & $\cdot 4108$ & $\cdot 4623$ & $\cdot 5122$ & 5611 \\
\hline$x=x^{\prime}$ & 5509 & $\cdot 4656$ & $\cdot 3794$ & $\cdot 2970$ & $\cdot 2187$ & $\cdot 1440$ & .0715 & 0.0 \\
\hline Sextic Oval, y & 0.0 & $\cdot 2218$ & $\cdot 2986$ & 3455 & 3754 & $\cdot 3941$ & $\cdot 4043$ & $\cdot 4075$ \\
\hline Ellipse, $\quad y^{\prime}$ & 0.0 & $\cdot 2178$ & $\cdot 2954$ & 3432 & $\cdot 3741$ & $\cdot 3932$ & $\cdot 4040$ & $\cdot 4075$ \\
\hline$\Delta=\left(y-y^{\prime}\right)$ & 0.0 & $\cdot 0040$ & .0032 & .0023 & .0013 & $\cdot 0009$ & $\cdot 0002$ & 0.0 \\
\hline
\end{tabular}


When the eccentricity is increased by giving a different value to $c$, the difference between the $y$ coordinates of the oval and the ellipse also increases, as shown in the following table, where $r_{02}=\cdot 9819 ; r_{01}=\cdot 0859 ; c=\cdot 4480 ; a=\cdot 5339 ; b=\cdot 3380$; other values of $r_{1} r_{2}$ being as above.

\begin{tabular}{|c|c|c|c|c|c|c|c|c|c|}
\hline$x=x^{\prime}$ & •5339 & .4743 & $\cdot 4009$ & $\cdot 3267$ & $\cdot 2577$ & $\cdot 1882$ & $\cdot 1240$ & .0615 & 0.0 \\
\hline$y$ & 0.0 & $\cdot 1634$ & $\cdot 2313$ & $\cdot 2733$ & 3007 & 3184 & 3298 & 3362 & $\cdot 3380$ \\
\hline$y^{\prime}$ & 0.0 & $\cdot 1550$ & $\cdot 2232$ & $\cdot 2670$ & $\cdot 2967$ & 3162 & $\cdot 3287$ & 3357 & .3380 \\
\hline$\Delta=\left(y-y^{\prime}\right)$ & 0.0 & .0084 & 0081 & .0063 & .0030 & .0022 & .0011 & .0005 & .0000 \\
\hline
\end{tabular}

In Pl. IV. fig. 6, the exterior curve of each pair represents the sextic oval, and the interior curve the ellipse, as traced through the points here given.

Further researches as to the properties of elliptic ovals may be expected to yield interesting results; and it appears to me that these curves, and the curves obtained from them by linear transformation, are capable of expressing the facts of a certain class of physical problems with greater accuracy than is obtainable by the tables at present in use for the purpose.

The three curves shown in Plate V. fig. 6, are linear transformations of the symmetrical oval. Their equation is $r_{1}^{6} / \lambda+r_{2}^{6} / \mu=1 ; \lambda$ was taken $=1$; and $\mu$ was taken successively $=2, \frac{1}{2}$ and $\frac{2}{3}$ to obtain the three curves. In the diagram each curve is connected with its foci by lines drawn for the purpose. The same equation with different values of either $c$ or $\mu$ gives a different curve, as the figure shows. The complete equation is

$$
\frac{r_{1}^{n}}{\lambda^{n}}+\frac{\mathrm{P} r_{1}^{n-2}}{\lambda^{n-2}} \frac{r_{2}^{2}}{\mu^{2}}++\cdots \frac{r_{2}^{n}}{\mu^{n}}=\mathrm{A}^{n}
$$

and the curve may be described as the Oval of single symmetry. The Cartesian oval is a limiting form of the oval of single symmetry of the 4 th degree, as may be verified by twice squaring its equation $r_{1} / \lambda+r_{2} / \mu=\mathrm{A}$.

The curves formed by giving negative values to one of the quantities, or to one of each pair of homologous terms, are remarkable for their varied and fantastic forms; but I have not been able to discover any properties which are common to the class.

The curve of the symmetrical equation $r_{2}^{6}-r_{1}^{6}=\mathrm{A}$ (shown in Pl. V. fig. 5) is an inflexional curve of two branches. Each branch crosses the axis of $\mathrm{X}$, and is symmetrical on either side of it; after being inflected on either side, the branches continue to approach to the asymptote $\mathrm{Y}$, which accordingly has double contact with the curve at the point infinity and also at negative infinity. Generally, for the symmetric radial equation of any number of terms of even powers, I have found (1) in the form $(\beta)$ or $(+--+)$, if the coefficients of the intermediate terms are fractional, the curve is an oval entirely concave to the centre; (2) if the coefficient (in the form $\beta$ ) exceed unity 
the curve is of the form last described (Pl. V. fig. 5), having the axis of $\mathrm{Y}$ for its asymptote; $(3)$ in the forms $(\epsilon)$ and $(\zeta)$, or $(+-+-)$ and $(++--)$, the curve is of the same form, having the axis of $Y$ for its asymptote. The linear transformation of the radial equation with negative terms has been found to be a closed curve in all the examples I have tried; it apparently only becomes asymptotic when the coefficients $\lambda$ and $\mu$ are equal. The oval and the mushroom-like forms of Plate V. fig. 7, are traced from the equation $r_{1}^{6} / \lambda-r_{2}^{6} / \mu=1$ by giving different values to $\mu$ and $c$.

It is easily seen that in the case of bi-radial curves with diverse coefficients, $\lambda$ and $\mu$, the transformation to polar coordinates will not give rise to a simplified expression, because the uneven terms of the expansions do not disappear. The equations in $x$ and $y$ contain in general all the even powers of $y$ and all the powers, even and uneven, of $x$, and are similar in form to those which have been considered as resulting from an oblique section of a central surface parallel to a plane through one of its axes of symmetry. 
LORD MOLAREN ON HOMOGENEOUS EQUATIONS-POSITIVE SYMMETRY.--PLATE I.

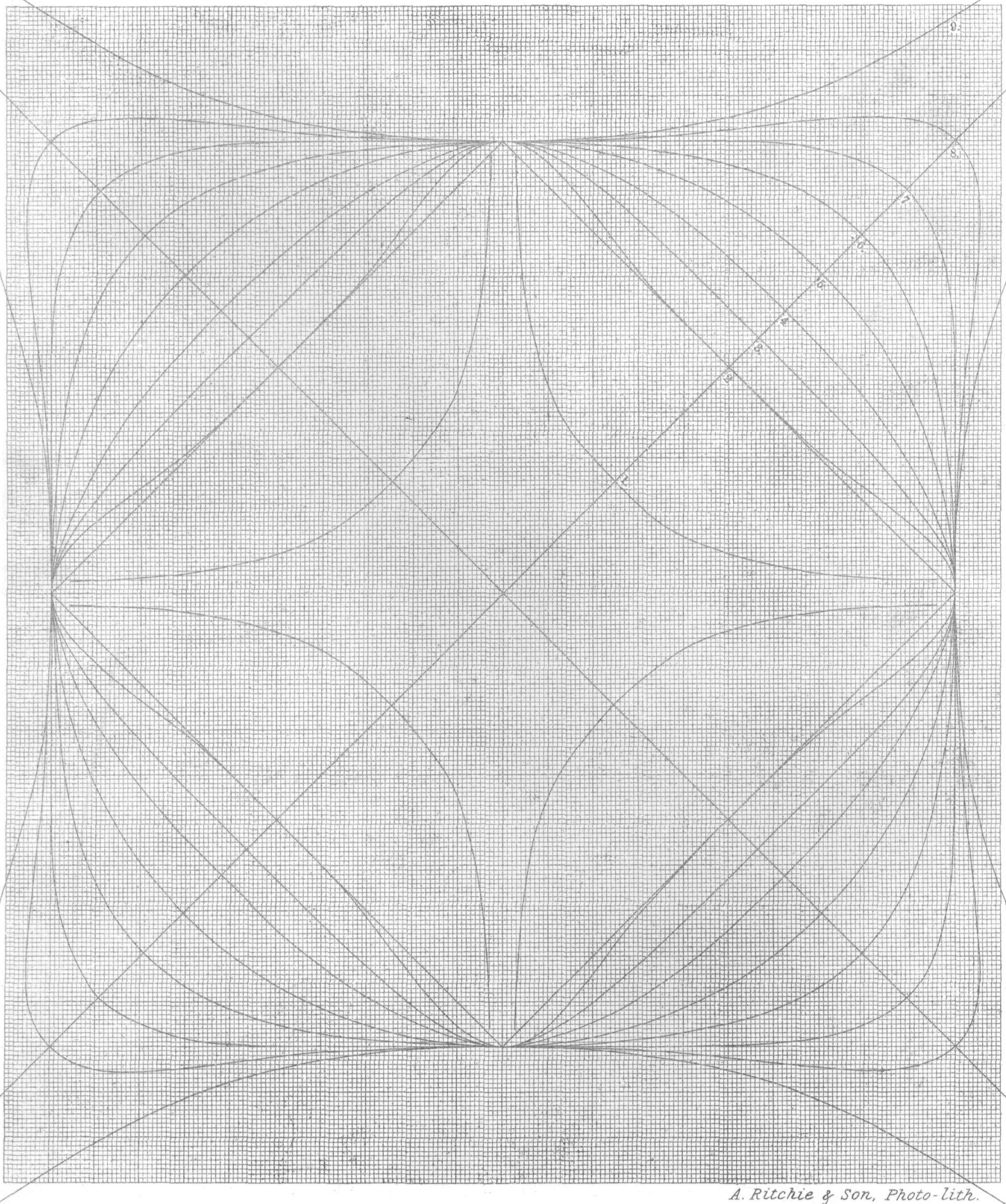


LORD MOLAREN ON HOMOGENEOUS EQUATIONS-NEGATIVE SYMMETRY.-PLATE. II.

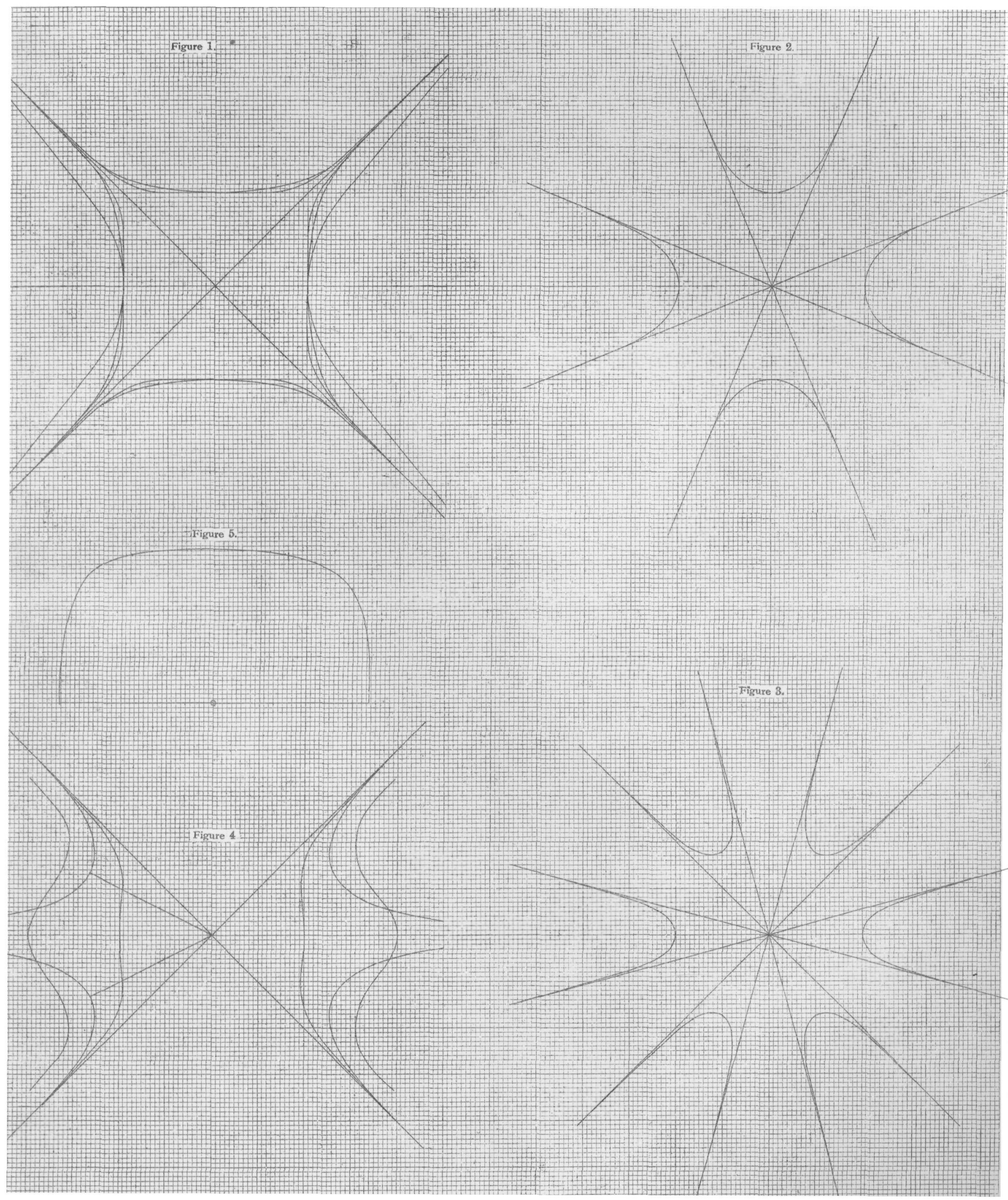


Trans. Roy. Soc. Edin. Vol.XXXV

LORD MCLAREN ON EQUATIONS OF TWO HOMOGENEOUS PARTS.-PLATE III.

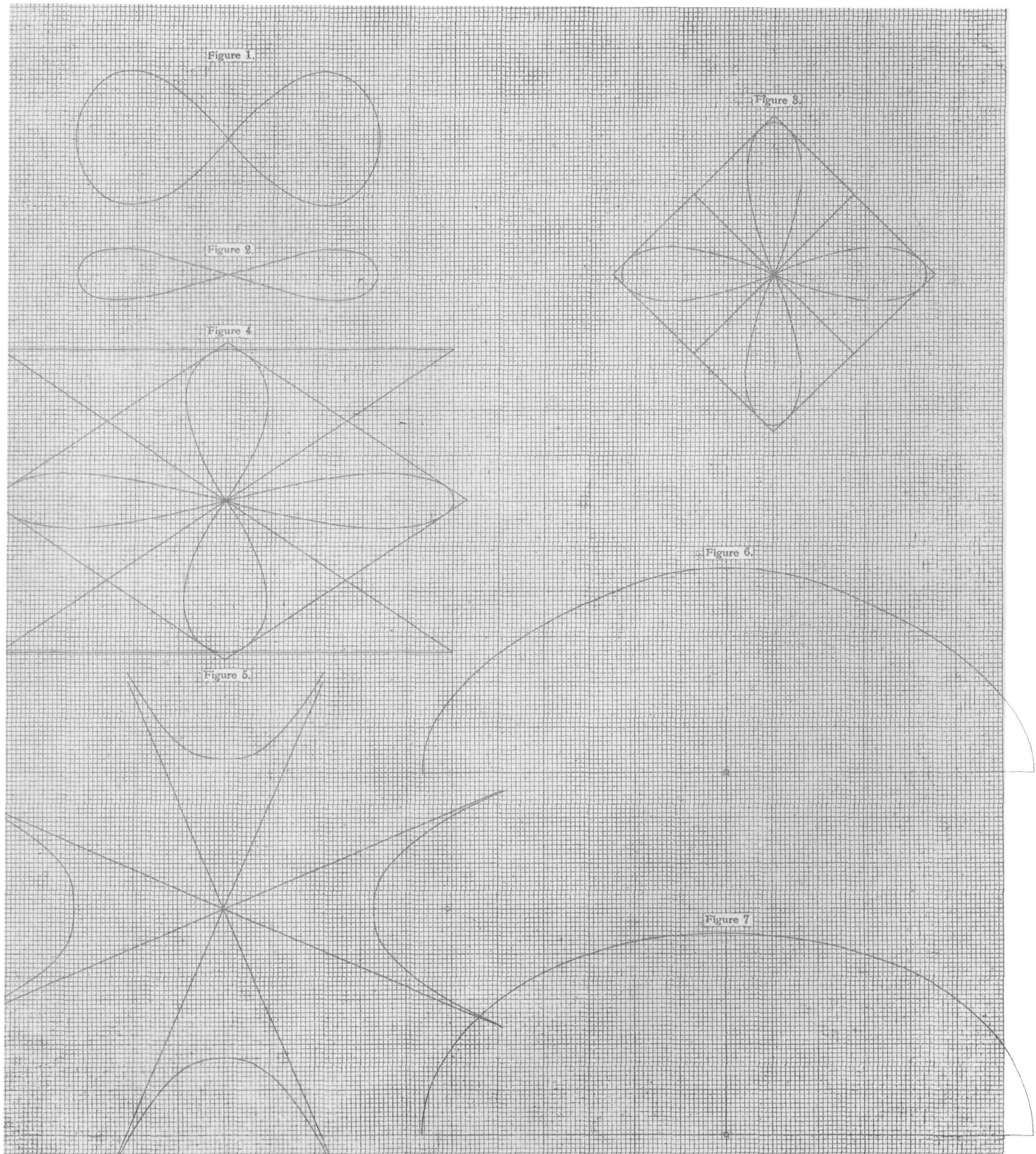

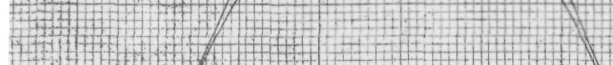


Trans. Roy. Soc. Edin. Vol.XXXV.

LORD MOLAREN ON EQUATIONS OF SINGLE, OR IMPERFECT SYMMETRY.--Plate IV.

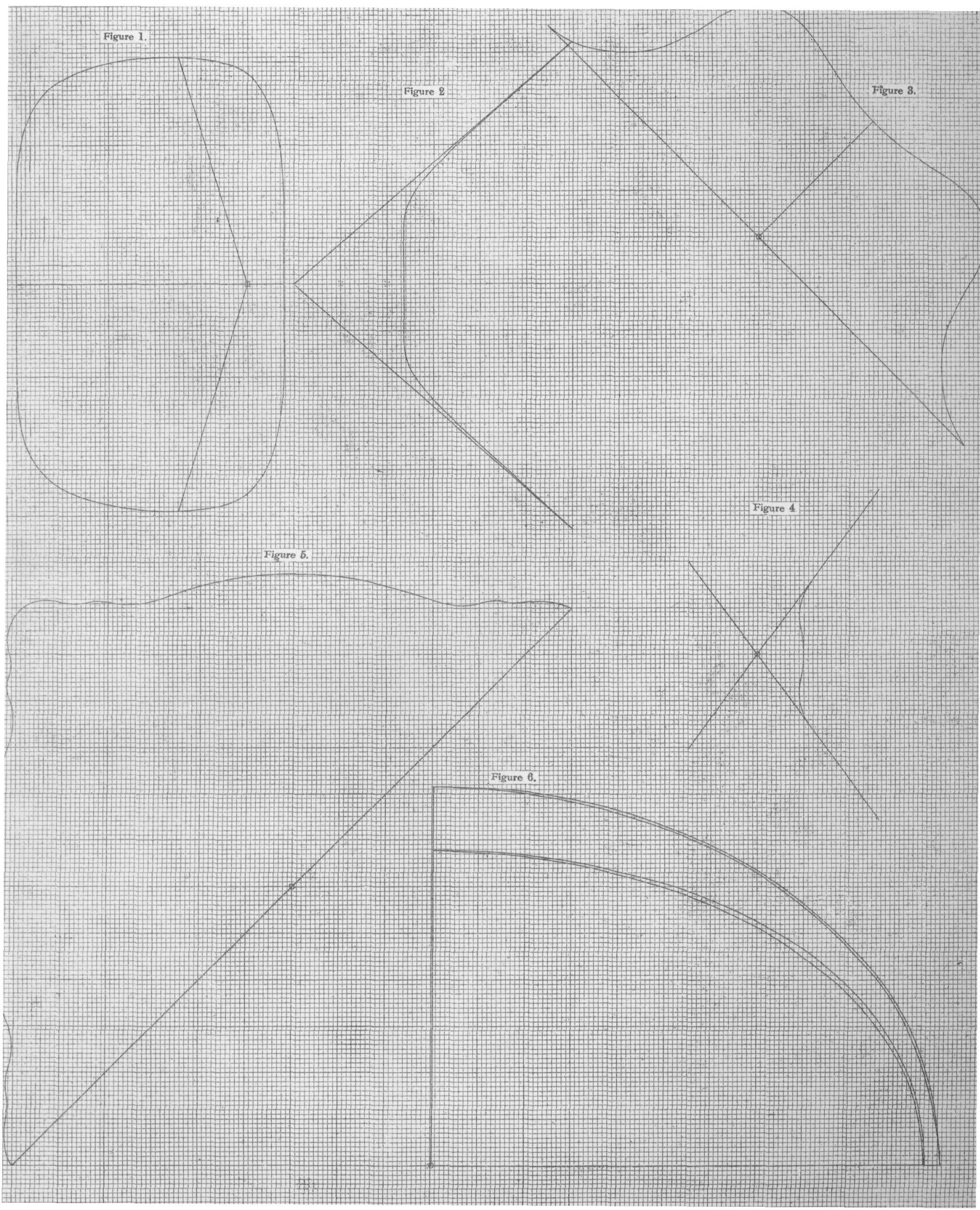




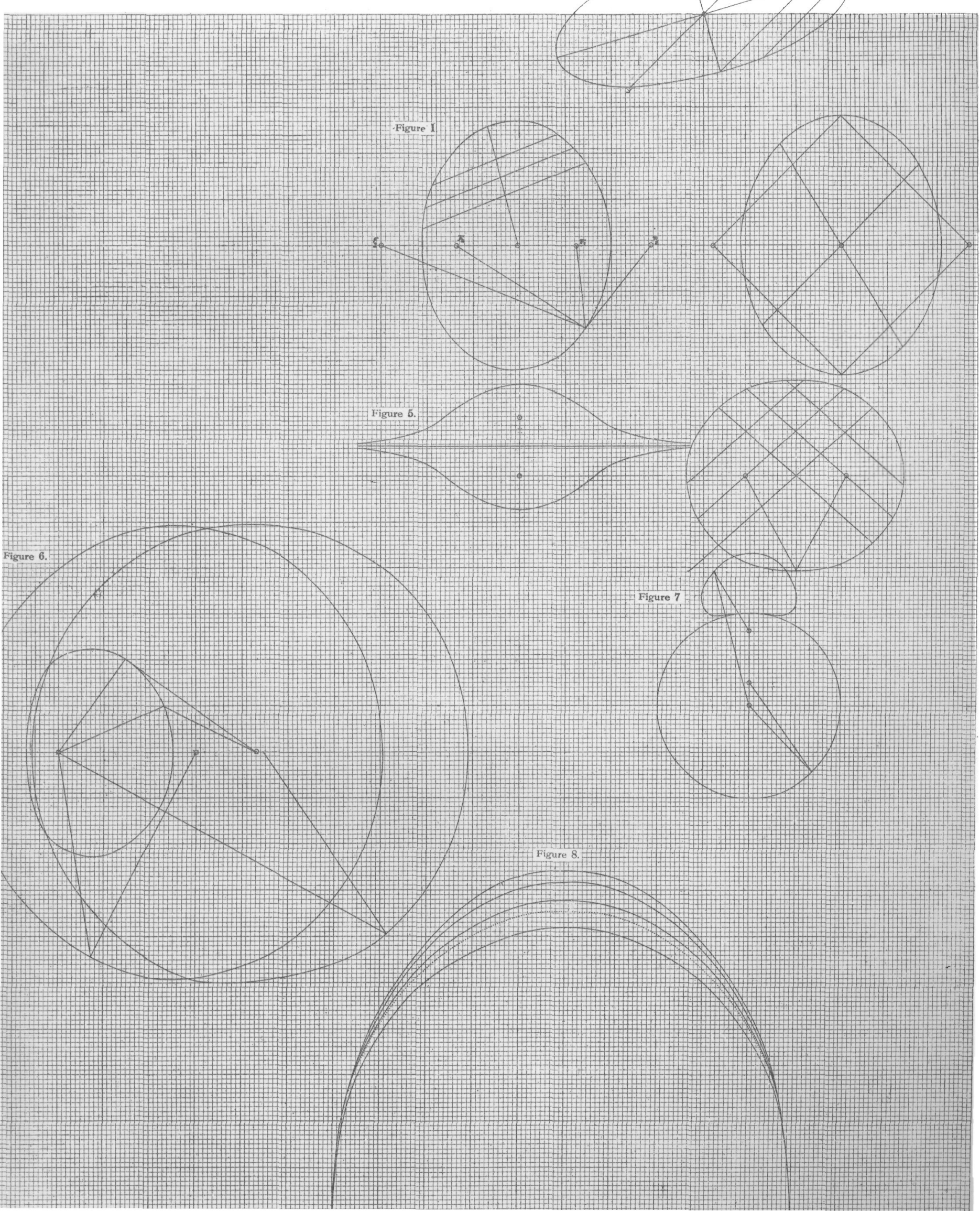


Trans. Roy. Soc. Edin. Vol.XXXV.

LORD MClaren ON CONTOUR-LINES OF SURFACES-HOMOGEneOUS EQUaTIONS.-Plate VI.

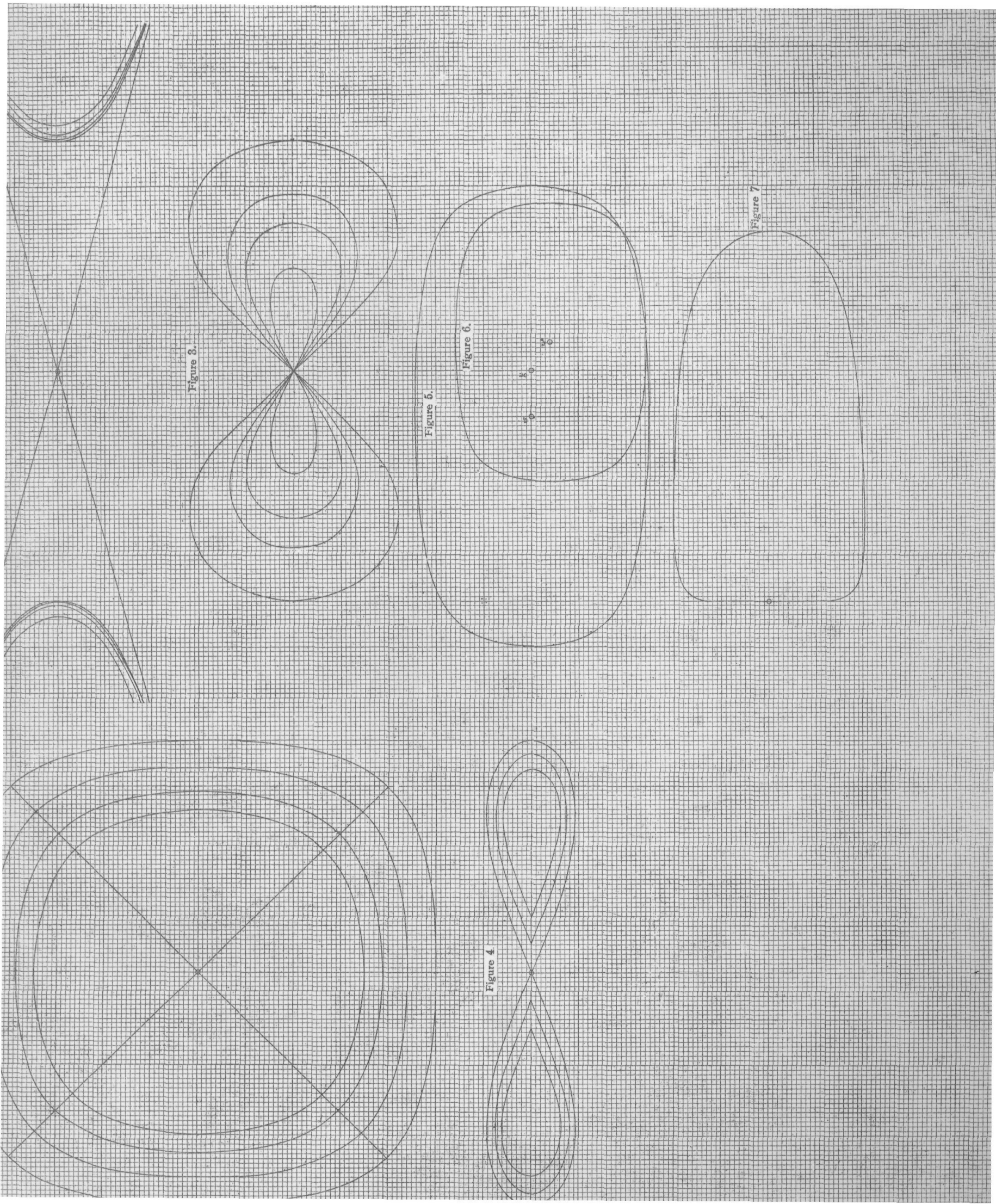

